\title{
WestVirginiaUniversity
}

THE RESEARCH REPOSITORY @ WVU

Graduate Theses, Dissertations, and Problem Reports

2011

\section{Response Elimination, Reinforcement Rate and Resurgence of Operant Behavior}

\author{
Carlos Renato Xavier Cancado \\ West Virginia University
}

Follow this and additional works at: https://researchrepository.wvu.edu/etd

\section{Recommended Citation}

Cancado, Carlos Renato Xavier, "Response Elimination, Reinforcement Rate and Resurgence of Operant Behavior" (2011). Graduate Theses, Dissertations, and Problem Reports. 3417.

https://researchrepository.wvu.edu/etd/3417

This Dissertation is protected by copyright and/or related rights. It has been brought to you by the The Research Repository @ WVU with permission from the rights-holder(s). You are free to use this Dissertation in any way that is permitted by the copyright and related rights legislation that applies to your use. For other uses you must obtain permission from the rights-holder(s) directly, unless additional rights are indicated by a Creative Commons license in the record and/ or on the work itself. This Dissertation has been accepted for inclusion in WVU Graduate Theses, Dissertations, and Problem Reports collection by an authorized administrator of The Research Repository @ WVU.

For more information, please contact researchrepository@mail.wvu.edu. 
Response Elimination, Reinforcement Rate and Resurgence of Operant Behavior

\author{
Carlos Renato Xavier Cançado
}

Dissertation submitted to the Eberly College of Arts and Sciences at West Virginia University in partial fulfillment of the requirements

for the degree of

Doctor of Philosophy

in

Psychology

\author{
Kennon A. Lattal, Ph.D., Chair \\ Michael Perone, Ph.D. \\ Gregory A. Lieving, Ph.D. \\ Elizabeth Kyonka, Ph.D. \\ Miranda Reed, Ph.D.
}

\title{
Department of Psychology Morgantown, West Virginia
} 2011

Keywords: Resurgence; Reinforcement Rate; Alternative Responding; Response Elimination; Behavioral History; Key Peck; Pigeons 


\begin{abstract}
Response Elimination, Reinforcement Rate and Resurgence of Operant Behavior

Carlos Renato Xavier Cançado
\end{abstract}

The effects of reinforcement rates of alternative responding on resurgence were studied in six experiments with pigeons. In Experiment 1A, key pecking was maintained on a multiple variable-interval (VI) VI schedule during the first, Training, phase. In the second, ResponseElimination, phase, variable differential-reinforcement-of-other-behavior (DRO) schedules were in effect in each component and reinforcement rates were equal and then, higher in one (rich) component, and lower in the other (lean), than in the Training phase. In the third, Resurgence, phase, reinforcers were discontinued and more resurgence occurred in the lean than in the rich component. Differences in Training-phase response rates between components also could have produced these results. In subsequent experiments, rich and lean components were those in which higher and lower response rates occurred in the Training phase, respectively. These experiments differed in how reinforcement rates were programmed during the ResponseElimination phase. Experiment 1B was a replication of Experiment 1A with experimentallynaïve pigeons. In Experiment 2, reinforcement rates were equal in one component and lower or higher in the other, relative to those programmed in the Training phase. In Experiment 3 , reinforcers were discontinued in each component before differential reinforcement rates were in effect. In Experiment 4, differential reinforcement rates were in effect initially and, then, reinforcement rates in each component were equal to those in the Training phase. In Experiment 5 , differential reinforcement rates were arranged by a fixed-DRO in each component and, in Experiment 6, alternative responding consisted of pecking a different key under VI schedules arranging higher and lower reinforcement rates than in the Training phase. Little to no resurgence occurred in each experiment, and differential resurgence was not systematically related to reinforcement rates of alternative responding. Schedule differences, topography of alternative responding, order of exposure to conditions or the length of Training and ResponseElimination phases could not explain these results, nor did current theoretical models of resurgence predict them. Reinforcement rates of alternative responding did not affect resurgence according to the schedule parameters in each experiment. Studies in which reinforcement rates of alternative responding were manipulated parametrically would clarify the present results and the effects of this variable on resurgence. 


\section{Acknowledgements}

The present experiments were conducted with the support of funds from the Doctoral Student Research Program and an Alumni Fund Award (May, 2010) of the Eberly College of Arts and Sciences at West Virginia University. Portions of these data were presented at the 2011 Annual Convention of the Association for Behavior Analysis International, Denver, CO.

I would like to thank my advisor, Andy Lattal, for his support and guidance, for teaching me how to ask questions about behavior and how to go about answering them. Thank you, Andy, for teaching me that we always have miles to run before sleeping (SR+)! I would also like to thank professor Mike Perone, who taught me how to look at an experiment, think about its important parts, understand it, and to plan better experiments. Andy and Mike, you are the examples of great researchers and teachers I will keep and always follow.

Professors Mike Perone, Gregory A. Lieving, Elizabeth Kyonka and Miranda Reed, thank you for accepting being committee members on my dissertation.

Many thanks to my friends, Andrew Lightner, Leigh Schrimpf, Anne Foreman, Toshikazu Kuroda, August Holtyn and Mirari Elcoro, for being there, always. Thank you Megan and Toquinho Maxwell, Carolina Aguilera and Lee Mahle, for you friendship and support. Professors Paulo Guerra and Carlos Eduardo Costa, thank you for being the great friends and audience that keeps me learning about behavior!

A special thank you to Professor Sérgio Dias Cirino, who one day suggested that I studied behavior analysis, and who always encouraged me to work and to make my dreams real. Sérgio, thank you for being an advisor, for introducing me to behavior analysis and for your friendship, which I will always honor.

Finally, I would like to thank my family, Suely, Carlos and Débora, Tita, Mingo, Dani and Clarisse for the love and support. Raquel, I have no words to say thank you. You were with me at all times, you bring joy to my life and gave me the three most precious things I will ever know: your love, our daughter, Elis, and another little person to share with us the great things about this world. 
Table of Contents

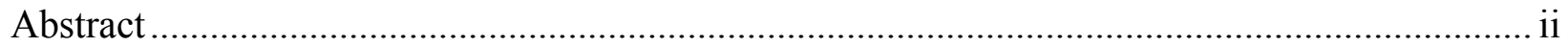

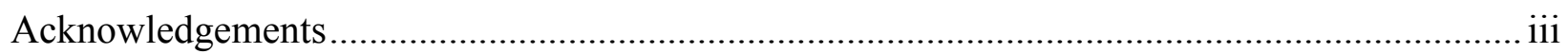

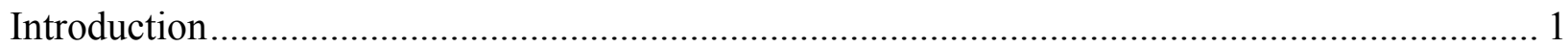

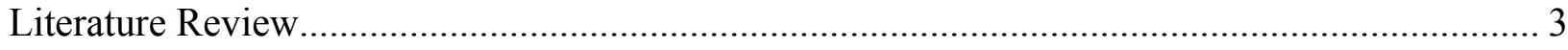

Resurgence and Rate of Reinforcement of Alternative Responding ..................................... 3

Resurgence, Schedules of Reinforcement and Topography of Alternative Responding.......... 11

Resurgence and Length of Training and Response-Elimination Phases .............................. 14

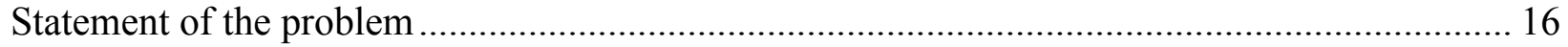

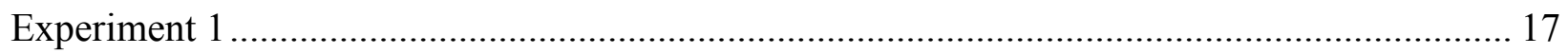

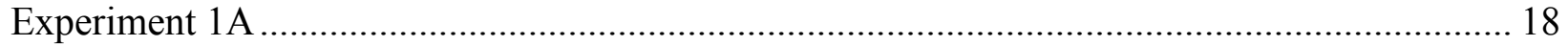

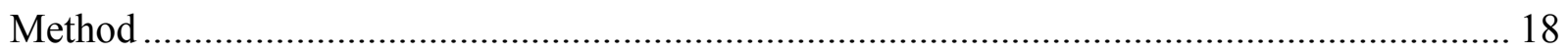

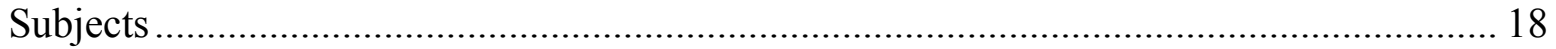

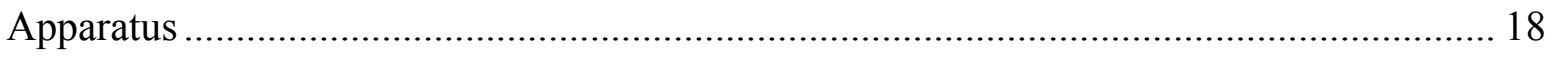

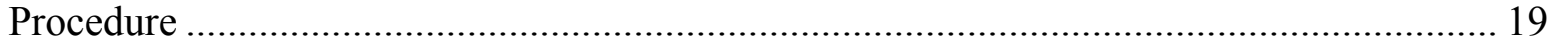

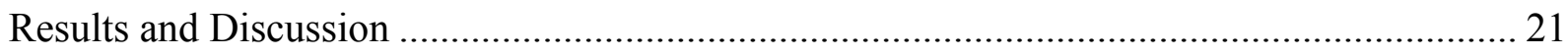

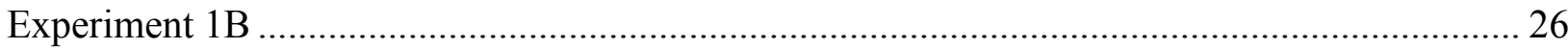

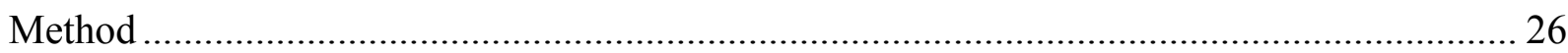

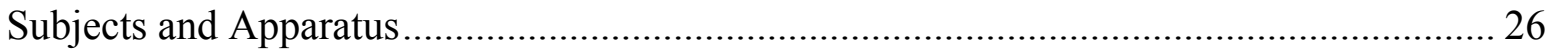

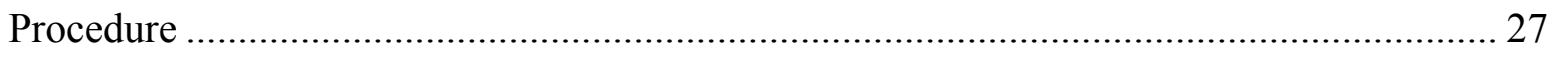

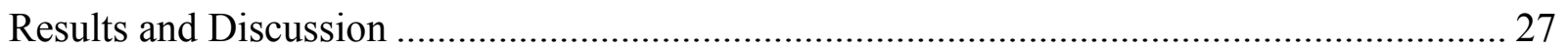

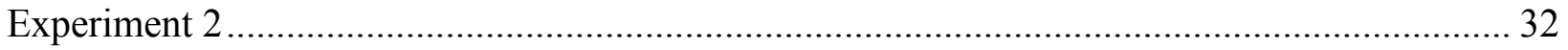

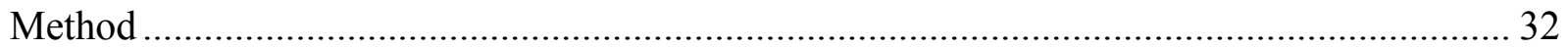

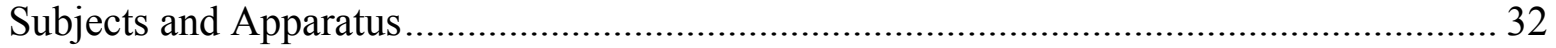

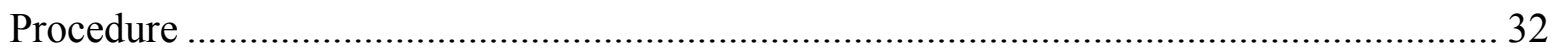

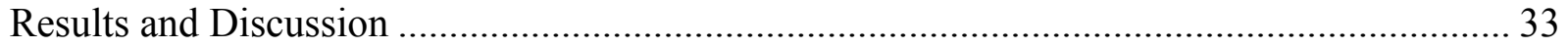

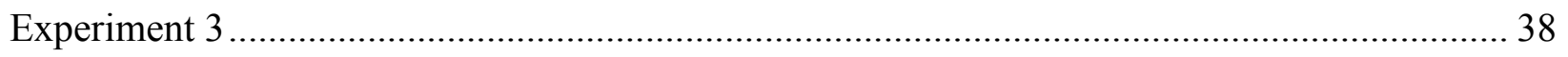

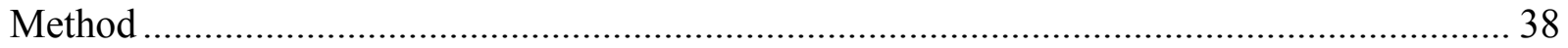

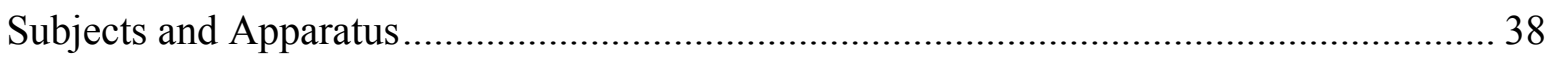

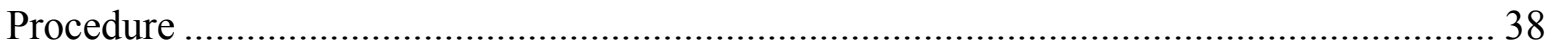

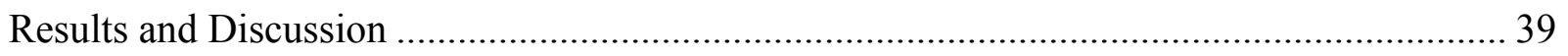

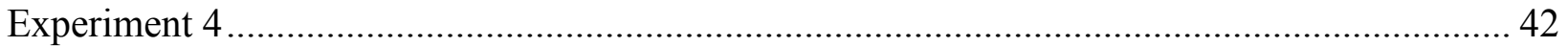

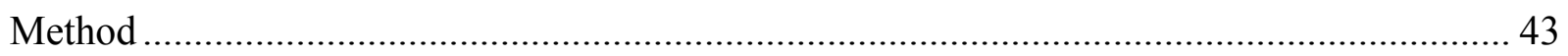

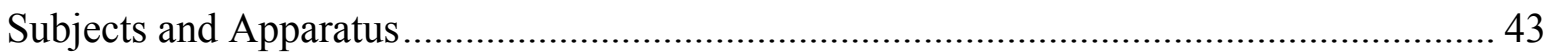

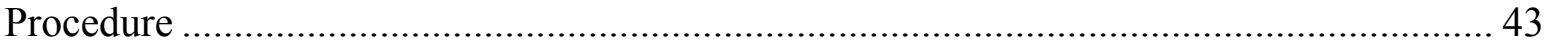

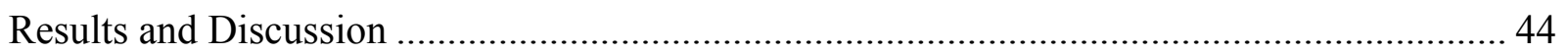




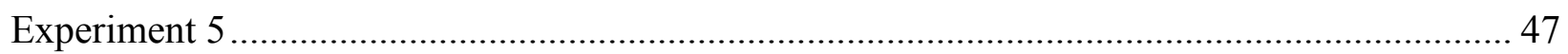

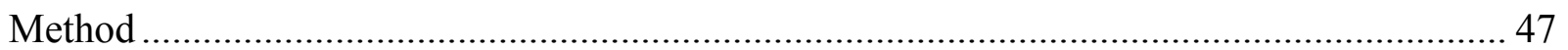

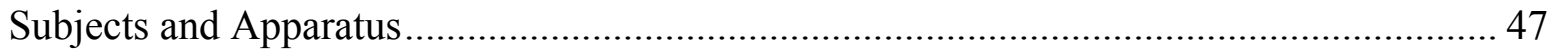

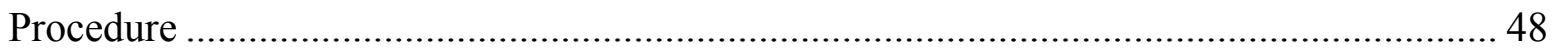

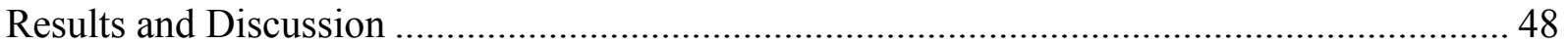

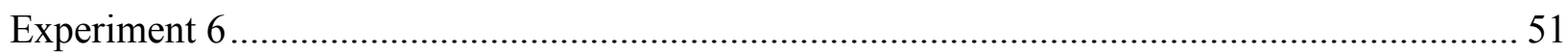

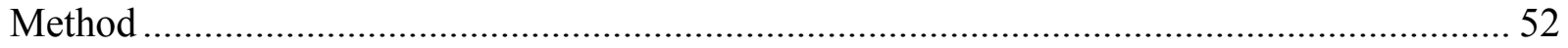

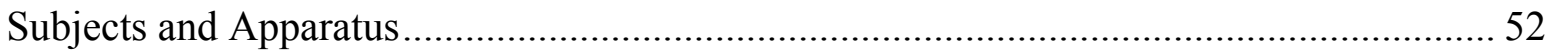

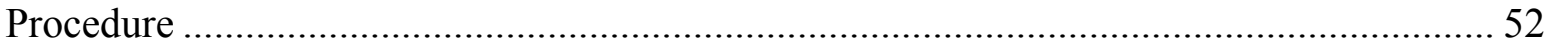

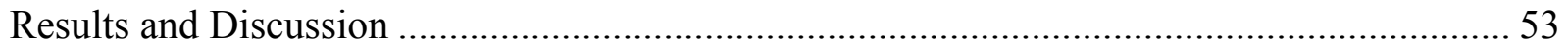

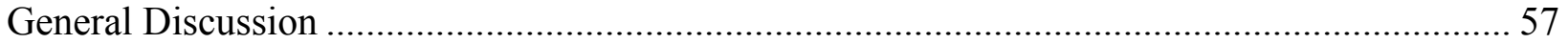

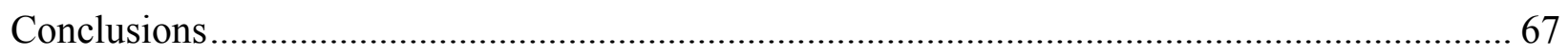

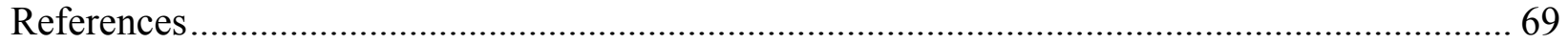

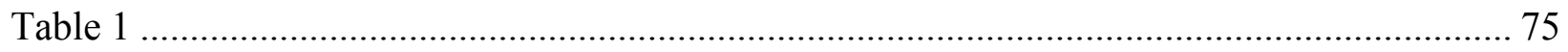

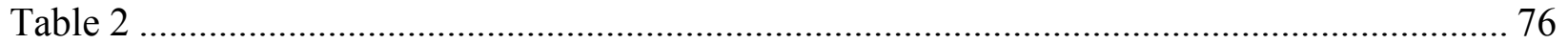

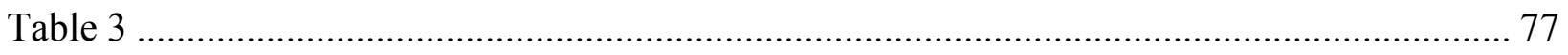

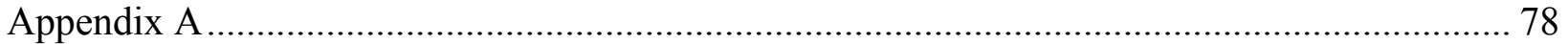

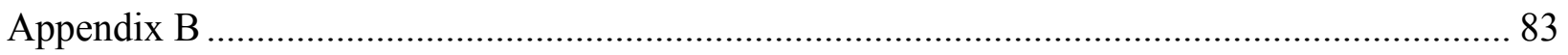

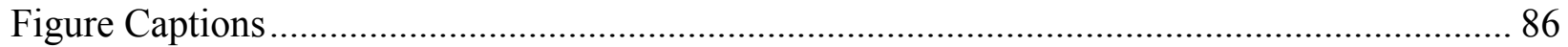

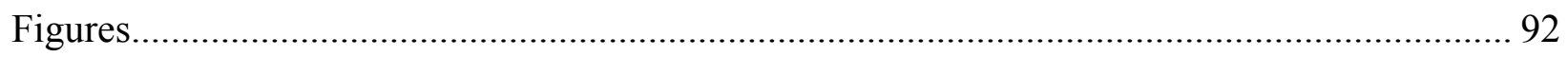




\section{Introduction}

Resurgence, the recurrence of previously reinforced responding when reinforcement for current responding is discontinued (Epstein, 1983, 1985), is studied by arranging three-phase procedures (Carey, 1951; Leitenberg, Rawson \& Bath 1970; Leitenberg, Rawson \& Mulick, 1975; Lieving \& Lattal, 2003). In the first, Training, phase, responding of a given form is reinforced. In the second, Response-Elimination, phase, an alternative response is reinforced and reinforcement for the first response is discontinued. In the third, Resurgence, phase, reinforcement for the alternative response also is discontinued. Resurgence is operationalized in this last phase as an increase in the frequency of the first response, relative to its frequency during the terminal sessions of the Response-Elimination phase (da Silva, Maxwell \& Lattal, 2008; Doughty, da Silva \& Lattal, 2007).

Doughty and Oken (2008) noted that the study of resurgence is useful to understanding extinction-induced responding more generally because it specifies the origins of such responding in an organism's behavioral history. In addition, it has been suggested that resurgence is involved in such behavioral phenomena as problem solving (Epstein, 1996; Shahan \& Chase, 2002) and clinical relapse (e.g., problem behavior; Bouton, 2011; Bruzek, Thompson \& Peters, 2009; Lieving, Hagopian, Long \& O’Connor, 2004; Volkert, Lerman, Call \& Trosclair-Lasserre, 2009; see also Thompson, Bruzek \& Cotnoir-Bichelman, 2011; and drug-maintained behavior; Podlesnik, Jimenez-Gomez \& Shahan, 2006).

Resurgence is a replicable phenomenon within subjects (Lieving \& Lattal, 2003), and previous studies indicate that variables operative during the Training, Response-Elimination and Resurgence phases affect the occurrence and the magnitude of resurgence (for reviews, see Doughty \& Oken, 2008; and Lattal \& St. Peter Pipkin, 2009). Particularly, rate of responding 
and rate of reinforcement in the Training phase, and also the schedules, the topography and the rate of reinforcement of alternative responding in the Response-Elimination phase have been shown to affect resurgence (e.g., da Silva et al., 2008; Doughty et al., 2007; Leitenberg et al., 1975; Lieving \& Lattal, 2003; Podlesnik \& Shahan, 2009, 2010; Reed \& Morgan, 2007). In general, more resurgence is observed when responding during the Training phase occurs at higher, rather than lower, rates (da Silva et al.), and also when it is maintained by relatively higher reinforcement rates (Podlesnik \& Shahan, 2009). In addition, Lieving and Lattal demonstrated that more resurgence is observed when reinforcers are discontinued in the Resurgence phase, relative to conditions in which low rates of food-delivery or other reinforcers are arranged in this phase (see their Experiments 3 and 4; see also Volkert et al., 2009).

The majority of studies on resurgence of discrete responses (e.g., da Silva et al., 2008; Lieving \& Lattal, 2003; Podlesnik \& Shahan, 2010) and also on resurgence of response sequences (e.g., Bachá-Mendez, Reid \& Mendoza-Soylovna, 2007; Cançado \& Lattal, 2011; Reed \& Morgan, 2006) focused on the effects on resurgence of responding during the Training phase (e.g., if occurring at high or low rates, and if consisting of spatial or temporal patterns of responding; da Silva et al.; Bachá-Mendez et al.; Cançado \& Lattal) and the conditions that maintained it (e.g., higher or lower reinforcement rates; da Silva et al.; Podlesnik \& Shahan). The effects on resurgence of characteristics of alternative responding and reinforcement rates in the Response-Elimination phase have been less systematically studied and, as described in the following literature review, the results have been inconsistent across studies (e.g., Mulick, Leitenberg \& Rawson, 1976; Leitenberg, et al., 1970; Leitenberg, et al., 1975; Reed \& Clark, 2011; Winterbauer \& Bouton, 2010). Thus, the purpose of the present experiments was to 
further assess the effects of reinforcement rate of alternative responding on resurgence of operant behavior.

\section{Literature Review}

Resurgence can be affected by the conditions in effect during the Response-Elimination phase. Specifically, the schedules of reinforcement in effect during this phase (e.g., Leitenberg, et al., 1970; Leitenberg et al., 1975; Pacitti \& Smith, 1977), the topography of alternative responses relative to responding established in the Training phase (e.g., Doughty et al., 2007; Rawson, Leitenberg, Mulick \& Lefebvre, 1977; but see Mulick, et al., 1976) and the rate of reinforcement of alternative responding (Leitenberg et al., 1975, Experiment 3; but see Reed \& Clark, 2011, and Winterbauer \& Bouton, 2010) have been described as variables that affect the occurrence and the magnitude of resurgence. Another variable previously suggested to affect resurgence is the length of the Training and Response-Elimination phases. More resurgence is observed, in general, when the Training and Response-Elimination phases are in effect for, respectively, longer and shorter periods (e.g., Bruzek et al., 2009; Doughty, Cash, Finch, Holloway, \& Wallington, 2010; Cleland, Foster \& Temple, 2000; Leitenberg et al., 1975, Experiment 4; but see Lieving \& Lattal, 2003, Experiment 1; and Reed \& Clark).

\section{Resurgence and Rate of Reinforcement of Alternative Responding}

In the 1970's, Leitenberg and colleagues reported a series of experiments conducted to assess the relative efficacy of different procedures for reducing the frequency of previously reinforced responding (e.g., Leitenberg et al., 1970; Leitenberg et al., 1975; Rawson \& Leitenberg, 1973; Rawson et al., 1977) and its recurrence when reinforcement for alternative responding was discontinued (in fact, the Resurgence phase in these studies was conducted to assess if the effects of different response-reduction procedures would be maintained in the 
absence of reinforcement for alternative responding; see especially Leitenberg et al., 1970). For example, in Leitenberg et al.'s (1975) Experiment 3, each of 30 pigeons was trained to key peck (Key A) on a VI 120-s for five sessions. During the Response-Elimination phase, extinction (EXT) of the first response was in effect for each pigeon, while pecking a different key (Key B) was maintained by relatively higher (VI 30 s), for one group, or lower (VI 240 s) reinforcement rates, for another group. Pigeons in a control group were not exposed to reinforcement of alternative responding during this phase. When reinforcers for alternative responding were discontinued, pecking Key A resurged more for the pigeons exposed to reinforcement of alternative responding during the Response-Elimination phase than for control-group pigeons. In addition, more resurgence occurred for the pigeons exposed to higher, rather than lower, rate of reinforcement of alternative responding.

Leitenberg et al. (1975; see also Leitenberg et al., 1970; Rawson \& Leitenberg, 1973; Rawson et al., 1977) argued that reinforcing alternative responding during the extinction of a previously reinforced response prevents the extinction of that response, which recurs when reinforcers for alternative responding are discontinued. That is, "temporary reinforcement of alternative behavior does not hasten the overall course of extinction, but merely suppresses the behavior to be extinguished and, thus, actually prevents extinction from taking place" (Leitenberg, et al., 1975, p. 641). This is a statement of the prevention-of-extinction hypothesis (or the response-prevention hypothesis; cf. Rawson et al., 1977) of resurgence. According to this account, resurgence would be inversely related to (a) the frequency of previously reinforced (i.e., Training) responding during the Response-Elimination phase (i.e., the more responding occurs in the absence of reinforcement, the higher the probability of extinction) and (b) the rate of reinforcement of alternative responding (i.e., higher reinforcement rates during the Response- 
Elimination phase would establish alternative responding with a higher probability, thus, preventing previously reinforced responding of contacting extinction). The prevention-ofextinction hypothesis cannot account for the occurrence of resurgence when responding is extinguished before reinforcement of alternative responding is in effect (e.g., of extinctioninduced resurgence; Bruzek et al., 2009; Epstein, 1983; Lieving \& Lattal, 2003) or even when differential-reinforcement-of-other behavior (DRO) schedules are in effect during the ResponseElimination phase (e.g., see especially da Silva et al., 2008; see also Doughty et al., 2007; Pacitti \& Smith, 1977).

The results obtained by Leitenberg et al. (1975; Experiment 3) for differential resurgence as a function of different reinforcement rates of alternative responding were not replicated consistently in series of experiments with rats conducted by Winterbauer and Bouton (2010), and also in an experiment by Reed and Clark (2011), in which individuals with autism served as participants. In Winterbauer and Bouton 's study, Training, Response-Elimination and Resurgence phases were conducted for a fixed number of 30-min sessions (5, 4, and 1session[s], respectively). In each experiment, rats first were trained to press one lever. During the Response-Elimination phase, this response was extinguished while pressing a different lever was reinforced. Across experiments, rats were exposed to schedules arranging an increase, a decrease, or no change in rate of reinforcement of alternative responding relative to the Training phase. For example, in their Experiment 1, a random-interval (RI) 30-s schedule was in effect in the Training phase. During the Response-Elimination phase, rats in one group were exposed to an RI 30 s (i.e., no change in programmed reinforcement rate) and those in another group to an RI 10 s (i.e., an increase in rate of reinforcement for alternative responding); rats in a control group were not exposed to reinforcement of alternative responding. Resurgence, assessed as the 
average number of Training-lever presses that occurred when reinforcers for alternative responding were discontinued, was similar for rats in each group in which alternative responding was reinforced.

In their Experiment 2, Winterbauer and Bouton (2010) reported results that were somewhat inconsistent with those of their first experiment. They used the same general procedure than in their Experiment 1, but an RI 10-s schedule was in effect during the Training phase for rats in each of three groups. In the Response Elimination-phase, alternative responding was reinforced according to an RI $10 \mathrm{~s}$, for one group, and according to an RI $30 \mathrm{~s}$, for the other (as in Experiment 1, the control-group rats received no reinforcement for alternative responses). Although more responding occurred during the Resurgence phase for rats exposed to higher (i.e., the RI $10 \mathrm{~s}$ ) than lower reinforcement rate of alternative responding, the mean number of responses differed only slightly between groups. The authors did not report statistical analyses comparing the results of these two groups, but only responding of each group with the control group, and also analyses comparing the group-mean number of responses of each group in the last sessions of the Response-Elimination and during the Resurgence phase. These analyses showed that resurgence occurred for groups exposed to the RI $10 \mathrm{~s}$ and RI $30 \mathrm{~s}$, but not that differences in resurgence between these groups occurred. In sum, the results of these two experiments reported by Winterbauer and Bouton suggested no systematic relation between reinforcement rate of alternative responding and resurgence.

That resurgence reliably occurred in each of four experiments in Winterbauer and Bouton's (2010) study, however, suggests that their findings are at odds with the prevention-ofextinction hypothesis (Leitenberg et al., 1970, 1975). That is, independently of the frequency of previously reinforced responding during the Response-Elimination phase (which, across studies, 
occurred consistently and was systematically reduced to zero or near zero relative to the Training phase), resurgence occurred when reinforcement rates during this phase decreased and also when it increased relative to reinforcement rates in the Training phase. According to the preventionof-extinction-hypothesis, more resurgence should have occurred when relatively higher rates of reinforcement of alternative responding were in effect during the Response-Elimination phase (i.e., suppressing the frequency of responding to the Training-phase operandum and preventing its contact with extinction), independent of the rates of reinforcement in effect during the Training phase (i.e., if arranged by an RI $30 \mathrm{~s}$ or $10 \mathrm{~s}$, as in Winterbauer and Bouton's Experiments 1 and 2).

Results that differ from those of Leitenberg et al. (1975) and Winterbauer and Bouton (2010) regarding the effects of reinforcement rates of alternative responding on resurgence were obtained by Reed and Clark (2011). Individuals with autism produced reinforcers (edibles) according to a variable-ratio (VR) 4 schedule for playing with a toy (Response 1) in a 30-min session. In the Response-Elimination phase, reinforcement for this first response was discontinued and, then, reinforcers for playing with a different toy (Response 2) were in effect. Participants were distributed in three groups during this phase. For one group, Response 2 was maintained on a VR 4 schedule, for a 30-min session and, for the other groups, a VR 2 and a VR 4 were in effect for, respectively, a 30- or 60-min session. During the Response-Elimination phase, reinforcement rate of alternative responding was similar for participants exposed to the VR 4 for 30 and $60 \mathrm{~min}$, respectively (and twice as lower than for participants exposed to the VR 2 for $30 \mathrm{~min}$ ), although the two groups exposed to the VR 4 differed in length and also in the total amount of reinforcers received. The groups exposed to the VR 2 and 4 for, respectively, 30 and $60 \mathrm{~min}$, differed in length, but were similar in the total amount of reinforcers received in this 
phase. Finally, the groups exposed to the VR 2 and 4 for 30 min were similar in length, but differed in the number of reinforcers received in this phase. In the Resurgence phase, which lasted for one, 5-min session, more resurgence occurred for the participants exposed to the VR 4 for 30 and $60 \mathrm{~min}$, than for those exposed to the VR 2 schedule. Thus, more resurgence occurred for participants exposed to relatively lower (i.e., VR 4 for 30 and $60 \mathrm{~min}$ ), rather than higher (VR 2 for a 30-min session) reinforcement rates of alternative responding during the ResponseElimination phase. It should be noted, however, that these differences in resurgence between groups were statistically significant for only the second minute of a single 5-min Resurgence phase, and no replications were reported in this study.

Although the results of these studies generally have been inconsistent regarding the effects of reinforcement rates of alternative responding on resurgence (e.g., Leitenberg et al., 1975; Winterbauer \& Bouton, 2010), some authors have proposed quantitative models that systematize findings of studies in which the effects of this variable on resurgence were studied. For example, Cleland, Guerin, Foster and Temple (2001) proposed a model of resurgence based on the generalized matching law (cf. Baum, 1973, 1974), which is a formal statement of the prevention-of-extinction hypothesis of resurgence (Leitenberg et al., 1975), but did not report experiments or simulations to test the predictions of their model and to assess how well it would described data from previous studies (see Appendix A for a detailed description of this model).

More recently, Shahan and Sweeney (2011; see Podlesnik and Shahan, 2009, 2010 for earlier versions of this model) proposed a quantitative model of resurgence based on behavioral momentum theory (cf. Nevin \& Grace, 2000; see Appendix A for a detailed description). This model assumes reinforcement rates of Training-phase and of alternative responding to be the main variables determining resurgence. It should be noted that Shahan and Sweeney assumed, 
procedurally, that the three-phase procedure was conducted such that reinforcement for alternative responding was in effect concurrently with the extinction of Training-phase responding (e.g., as in Leitenberg et al.'s study, but different from studies in which Trainingphase responding is extinguished before alternative responding is reinforced, e.g., Epstein, 1983; Bruzek, et al., 2009).

Shahan and Sweeney's (2011) model predicts more resurgence when responding is maintained by higher, rather than lower, reinforcement rates during the Training phase (as reported by Podlesnik \& Shahan, 2009, 2010; but see da Silva et al., 2008). The main assumption of the model, however, is that reinforcement of alternative responding during the Response-Elimination phase disrupts the occurrence of previously reinforced responding (i.e., Training-phase responding) but also increases its strength (cf. Nevin \& Grace, 2000; by the correlation between a discriminative stimulus context and reinforcement) and subsequent resurgence when reinforcement for alternative responding is discontinued in the Resurgence phase. Thus, more resurgence is also predicted when higher, rather than lower rates of reinforcement of alternative responding are in effect during the Response-Elimination phase. Shahan and Sweeney tested the predictions of their model by reanalyzing data from studies in which the rate of reinforcement of alternative responding (a) was manipulated between subjects (e.g., Leitenberg et al., 1975; Experiment 3) and (b) was not manipulated, and the effects on resurgence of reinforcement rates during the Training phase were assessed by within-subject comparisons (e.g., Podlesnik \& Shahan, 2010). In both cases, despite this methodological difference, the model accounted for more than $90 \%$ of the variance in the data.

In the absence of data from experiments, Shahan and Sweeney (2011) reported simulations of responding during the Resurgence phase, based on their model, in which 
reinforcement rate of alternative responding was varied parametrically (see especially their Figure 4, p. 100). In these simulations, reinforcement rates during the Training phase were arranged by a multiple VI 30 s VI 120 s and, in the Response-Elimination phase, rate of reinforcement of alternative responding in each component was programmed according to equal VI schedules, the parameters of which were varied between $240 \mathrm{~s}$ (relatively lean) to $15 \mathrm{~s}$ (relatively rich). The model always predicted more resurgence in the rich than in the lean component. Increasing the rate of reinforcement of alternative responding, in addition to increasing the magnitude of resurgence, decreased the differences in responding between schedule components in the Resurgence phase. That is, the degree of differential resurgence, according to their model, also would be a function of the parameters of the schedules of reinforcement in effect during the Training and Response-Elimination phases (see Figures A1 and A2 in Appendix A for a simulation of responding based on schedule parameters in effect in the present study).

Thus, although these quantitative models of resurgence (Cleland et al., 2001; Shahan \& Sweeney, 2011) and the results of some studies previously described in this section indicate that resurgence can be affected by reinforcement rate of alternative responding, the results across studies have been inconsistent (Leitenberg et al., 1975, Experiment 3; Reed \& Clark, 2011; Winterbauer \& Bouton, 2010, Experiments 1 and 2). That is, more resurgence has been described when relatively higher reinforcement rates of alternative responding were in effect during the Response-Elimination phase (Leitenberg et al., Experiment 3; Winterbauer \& Bouton, Experiment 2), but also when a relatively lower frequency of reinforcement was in effect during this phase (Reed \& Clark). In addition, Winterbauer and Bouton (Experiment 1) reported no systematic relation between resurgence and rate of reinforcement of alternative responding. 
These inconsistencies provided the basis for further analyses of the effects of reinforcement rate of alternative responding on resurgence conducted in the present study.

\section{Resurgence, Schedules of Reinforcement and Topography of Alternative Responding}

The effects on resurgence of different schedules of reinforcement, and of the topography of alternative responding, during the Response-Elimination phase have been assessed previously (Leitenberg et al., 1975; Experiment 2; Winterbauer \& Bouton, 2010; Experiment 4; Pacitti \& Smith, 1977; Mulick et al., 1976; Doughty et al., 2007). In some studies, differential resurgence was reported between or within subjects when different schedules and response topographies were in effect during the Response-Elimination phase. In others, however, the occurrence and extent of resurgence was not affected by these variables.

Leitenberg et al. (1975) suggested that resurgence is not affected by the schedule of reinforcement in effect in the Response-Elimination phase, given that reinforcement rates in this phase are equal to those obtained in the Training phase. In their Experiment 2, rats first were trained to lever press and, during the Response-Elimination phase, were divided into three groups. In this phase, reinforcers for the first-trained response were discontinued and, for one group, pressing a different lever was reinforced according to a fixed-ratio (FR) schedule. For a second group, a yoked-VI schedule was in effect such that the IRIs were equated between FR and VI schedules. No reinforcers for alternative responding occurred for the third, control, group. More resurgence occurred for the rats exposed to either of the schedules of alternative reinforcement relative to that which occurred in the control group animals. In addition, no differences in resurgence occurred between the groups exposed to the FR or VI schedules. Winterbauer and Bouton (2010; Experiment 4) used a similar procedure to that of Leitenberg et al. and replicated their results. An additional group, however, was exposed to a VT schedule 
yoked to the FR schedule in effect for one of the other groups such that food-deliveries occurred at a rate similar to that for the other groups during the Response-Elimination phase. Resurgence was similar for the three groups.

In contrast, the schedules of reinforcement and the topography of alternative responding during the Response-Elimination phase have been considered to affect resurgence. For example, Pacitti and Smith (1977) conducted a study with rats in which resurgence was assessed after four different methods of eliminating lever-pressing responses previously maintained by a VI schedule. In the Response-Elimination phase, responding of one group was extinguished, and another was exposed to a DRO 20-s schedule. Responding of the third and fourth groups was reinforced on an FR 10 schedule of, respectively, pressing a different lever or pushing a vertical pole. When reinforcers were discontinued in the Resurgence phase, resurgence occurred for rats in each of the groups exposed to alternative reinforcement schedules relative to the group exposed to extinction. More resurgence was observed for the rats exposed to the FR schedule (either for lever pressing or pole pushing) than for those exposed to the DRO schedule. One limitation of this study, however, is that rate of reinforcement of alternative responding was not equated between groups (as it was in the studies by Leitenberg et al., 1975; and Winterbauer \& Bouton, 2010). As a result, differences in resurgence cannot be attributed solely to differences in schedules of reinforcement in effect in their study (cf. Doughty et al., 2007).

In the previously described experiments by Leitenberg et al., 1975, Winterbauer and Bouton (2010) and Pacitti and Smith (1977), comparisons of the effects of schedules of reinforcement and topography of alternative responding on resurgence were between groups. Mulick et al. (1976) conducted a similar analysis using a within-subject design. Squirrel monkeys were trained to lever press on a three-component multiple schedule. During the 
Training phase, a VI $30 \mathrm{~s}$ was in effect in each component. In the Response-Elimination phase, extinction was in effect in one component, a DRO 20- or 30-s schedule in the second, and a fixed-interval (FI) 20- or 30-s schedule for pressing a different lever was in effect in the third component. In their Experiment 1, little to no resurgence occurred in each schedule component and was not differential between components. Similar results were obtained in their Experiment 2, in which a VI schedule remained in effect in one component during Training, ResponseElimination and Resurgence phases, but the other two components were as in their Experiment 1. Doughty et al., (2007) conducted a series of experiments with pigeons addressing the same questions as Pacitti and Smith (1977) and Mulick et al. (1976), but using a two-component multiple schedule and equating reinforcement rates between components across Training and Response-Elimination phases. Doughty et al. suggested that, during the Response-Elimination phase, reinforcing responding that is topographically different from that established during the Training phase produces more resurgence than when Training- and Response-Elimination phase responding are similar in topography. In their study, key pecking first was maintained on VI 30s schedules in each schedule component. Across experiments, variable- or fixed-DRO schedules, and VI schedules of reinforcement of either pecking a different key or of pressing a treadle, were in effect in each component during the Response-Elimination phase. Resurgence of greater magnitude and earlier onset occurred in the component correlated with either DRO schedules relative to pecking different key. In their Experiment 4, there were no differences in resurgence when DRO schedules and VI schedules correlated with a different key were in effect in the two components during the Response-Elimination phase. Thus, different from Pacitti and Smith and Mulick et al., Doughty et al. found consistently more resurgence when DRO 
schedules were in effect during the Response-Elimination phase, relative to other schedules of reinforcement and topographies of alternative responding.

\section{Resurgence and Length of Training and Response-Elimination Phases}

In general, more resurgence occurs of responding with longer exposure to the conditions in effect during the Training phase. Bruzek et al. (2009) showed this effect in a study with college students in which resurgence of responding previously maintained by negative reinforcement was assessed. A simulated caregiving situation was arranged in which a recorded infant cry sounded and could be terminated contingent upon the participants' responses towards a baby doll (e.g., playing with a toy, rocking). In their Experiment 2, different responses were trained sequentially such that the first trained response had a longer history of reinforcement than did the second. These responses then were extinguished, after which a third, different, response was reinforced. In the Resurgence phase, all responses were ineffective in terminating crying. For five of eight participants, more resurgence was observed for responses that had longer, but temporally more distant, histories of reinforcement.

These results were replicated by Doughty et al. (2010) in a study in which college students were trained on two four-choice arbitrary-matching-to-sample tasks, presented in trials that occurred during each component of a multiple schedule. In each trial, one sample and four comparison stimuli were presented on a computer screen. On AB trials, selecting B1 in the presence of $\mathrm{A} 1$, and $\mathrm{B} 2$ in the presence of $\mathrm{A} 2$ produced points; on $\mathrm{CD}$ trials, selecting D1 and D2 produced points in the presence of, respectively, $\mathrm{C} 1$ and $\mathrm{C} 2$. Although relatively more $\mathrm{AB}$ trials occurred for each participant, at the end of training no differences in performance were observed across participants between $\mathrm{AB}$ and $\mathrm{CD}$ trials. Next, in the presence of $\mathrm{A} 2$ and $\mathrm{C} 2$ points were earned only when selecting, respectively, B3 and D3. In the Resurgence phase, reinforcers were 
discontinued for this recently trained discrimination and, as in Bruzek et al.'s study, more resurgence of the discrimination with a longer history of reinforcement (selecting B2 in the presence of A2) occurred for each participant.

The effects of the length of the Response-Elimination phase on resurgence are mixed. Leitenberg et al. (1975; Experiment 4) found that resurgence of lever pressing by rats in different groups was inversely related to the length of the Response-Elimination phase (3, 9 or 27 days), during which reinforcers for the first-trained lever press were discontinued and pressing a different lever was reinforced. Cleland et al. (2000) obtained similar results with hens. In the Response-Elimination phase of their study, reinforcement of alternative responses (e.g., head bobbing or door pushing) was in effect immediately after the Training phase, or after nine sessions in which reinforcers for Training-phase responding were discontinued. More resurgence occurred consistently when reinforcement for alternative responding started immediately after the Training phase (see Appendix A for an account of the effects of the length of the Response-Elimination phase on resurgence made by Shahan \& Sweeney, 2011). By contrast, Lieving and Lattal (2003; Experiment 1) reported that resurgence of pigeons' key pecking was not differentially affected when the Response-Elimination phase consisted of 10 , 30-min, sessions in which the frequency of key pecking was reduced to zero followed by either 5 or 30 sessions in which treadle pressing was reinforced according to a VI $30 \mathrm{~s}$. As described previously, Reed and Clark (2011) also found no effects of length of the Response-Elimination phase on resurgence after either 30- or 60-min sessions during which reinforcement of alternative responding was in effect.

Even though the effects of the length of the Response-Elimination phase on resurgence are ambiguous, they should be considered in the design of experiments because such length can 
be cofounded with the effects of other variables of interest (e.g., reinforcement rates). This can occur especially because it is common to conduct the Response- Elimination phase for a minimum number of sessions and until rates of previously reinforced responding are zero or near zero (e.g., da Silva et al., 2008; Podlesnik \& Shahan, 2009), so that its length may vary between subjects. Thus, as inconsistent results regarding the length of the Response-Elimination phase on resurgence exist, care should be taken not to expose subjects to an unnecessarily large number of sessions (maybe precluding observations of resurgence) and also to control for amount of exposure to different conditions in effect during this phase, when it is of interest to compare their effects.

\section{Statement of the problem}

Lengthier Response-Elimination phases apparently reduce the probability of resurgence (Cleland et al., 2000; Leitenberg et al., 1975, Experiment 4), although no systematic effects of this variable on resurgence also have been reported (Lieving \& Lattal, 2003, Experiment 1; Reed \& Clark, 2011). Inconsistent results also have been observed in studies assessing the effects on resurgence of different schedules of reinforcement, and the topography of alternative responding, in the Response-Elimination phase. In some studies, resurgence was affected by differences in the schedules of reinforcement and in the topography of alternative responding (e.g., Doughty et al., 2007; Pacitti \& Smith, 1977). These variables had no effect on resurgence in other studies (e.g., Leitenberg et al., Experiment 2; Mulick et al, 1976; Winterbauer \& Bouton, 2010, Experiment 4). Finally, contrasting results also have been found when reinforcement rates of alternative responding have been manipulated. Specifically, more resurgence has been reported when higher, rather than lower reinforcement rates of alternative responding are in effect in the Response-Elimination phase (Leitenberg et al., Experiment 3), but opposite results, and even no 
differences in resurgence as a function of this variable, also were reported previously (Reed \& Clark; Winterbauer \& Bouton, Experiments 1 and 2).

Given these inconsistencies in the results of previous studies, the present series of experiments was conducted to further investigate the effects reinforcement rates of alternative responding on resurgence. Relative to the programmed reinforcement rates during the Training phase, higher and lower reinforcement rates of alternative responding were in effect in the Response-Elimination phase, which had a fixed duration in each experiment to control for the length of exposure to this phase. The procedure used to arrange differential rates of reinforcement of alternative responding differed in each of the present experiments (see Table 1 for a summary of schedules of reinforcement and schedule parameters in effect during the Response-Elimination phase of each experiment; see description of each experiment below). Finally, the effects of reinforcement rates of alternative responding on resurgence were studied previously by using between-subject comparisons (Leitenberg et al., 1975; Winterbauer \& Bouton, 2010) and quantitative models of resurgence have been largely based on data from these studies (particularly on the results of Leitenberg et al., 1975, Experiment 3; see Cleland et al., 2001; Shahan \& Sweeney, 2011, and Appendix A). Thus, it was also a goal of the present experiments to extend these previous studies by using two-component multiple schedules of reinforcement and within-subject comparisons of resurgence as a function of differential reinforcement rates of alternative responding during the Response-Elimination phase.

\section{Experiment 1}

This experiment was conducted to assess if differential resurgence (cf. da Silva et al., 2008; Doughty et al., 2007) of previously reinforced key pecking by pigeons would occur when alternative responding was maintained by higher or lower reinforcement rates relative to those 
programmed during the Training phase. Experiment $1 \mathrm{~A}$ was conducted with four pigeons with previous exposure to various schedules of food-reinforcement. Experiment 1B was a replication of that experiment with four experimentally-naïve pigeons.

\section{Experiment 1A}

\section{Method}

\section{Subjects}

Three male and one female (627) White Carneau pigeons with previous exposure to various schedules of food-reinforcement served. Each was maintained at $80 \%( \pm 15 \mathrm{~g})$ of its free-feeding body weight and housed individually, with free access to water and health grit, in a colony room with a $12 \mathrm{hr}: 12 \mathrm{hr}$ light: dark cycle (lights on at 7:00 am).

\section{Apparatus}

Four operant chambers (30-cm long x 32-cm wide x 38-cm high) located in sound attenuating enclosures were used. For three chambers, the front wall was an aluminum panel with three 2-cm diameter Gerbrands Co. response keys, $9 \mathrm{~cm}$ apart (center to center) and with their lower edge $25 \mathrm{~cm}$ from the floor. The center key was used, and could be transilluminated red or amber, in one chamber, and red or green, in the other two chambers, and operated by a minimum force of $0.15 \mathrm{~N}$. The front panel of the fourth chamber contained two response keys, and the left key, which was used, could also be transilluminated red or amber. General illumination was provided by two $28-\mathrm{V}$ white houselights located in the lower right corner of the front panel for three chambers and in the upper right corner for the fourth. A food hopper, located behind a rectangular aperture $(5 \mathrm{~cm} \mathrm{x} 4 \mathrm{~cm})$ at the center of the front panel, with its lower edge $8 \mathrm{~cm}$ from the floor, was illuminated by a $28-\mathrm{V}$ DC white light and provided 3-s access to mixed grain when raised. The houselight and the keylights were off when the hopper was raised. 
White noise and a ventilation fan were used in each chamber to mask extraneous sounds.

Programming of conditions and data recording were accomplished by using MED-PC® software and interface and a Dell® computer located in an adjacent room.

\section{Procedure}

Due to each pigeon's experimental histories, no pretraining was required. A twocomponent multiple schedule of reinforcement was in effect in each phase and conditions were a replication of the general procedure described by Doughty et al. (2007). For each pigeon, one schedule component was correlated with a red keylight, and the other with a green (Pigeons 617 and 681) or amber (Pigeons 627 and 580) keylight. Table 2 shows, for each pigeon in this and in the subsequent experiments, the keylight colors correlated with each schedule component during the Response-Elimination phase. Sessions started after 180-s blackouts, during which the houselight and the keylight were off. The first component in a session was selected semirandomly such that no more than three consecutive sessions started with the same component. Thereafter, components alternated strictly after $180 \mathrm{~s}$ (excluding reinforcement time), each being presented five times in a session, and were separated by 30 -s intercomponent intervals (ICIs), during which the houselight and the keylight were off.

When either component was in effect, the keylight (see Table 2) and the houselight were on. In the Training and Response-Elimination phases (see descriptions of each phase below) reinforcers were arranged according to variable schedules (VI and DRO schedules of reinforcement, cf. Doughty et al., 2007, respectively). These schedules consisted of 15 intervals, derived from Fleshler and Hoffman's (1962) distribution. Intervals were selected randomly without replacement, during each session. Sessions were conducted seven days a week, at approximately the same time each day, during the light period of the colony room light: dark 
cycle. The schedules of reinforcement, stimulus conditions and stability criteria during each phase are described below.

Training. In this phase, a VI 30-s schedule was in effect in each component of the multiple schedule. This schedule parameter was selected to maintain moderate rates of key pecking and to program a reinforcement rate of two reinforcers per minute in each schedule component. Reinforcers programmed but not collected in either component were carried over to the next presentation of the same component.

This phase lasted for a minimum of 20 sessions and until responding was stable in each component. Responding was judged stable when (a) the difference between mean response rates of the last three and the immediately preceding three sessions was less than $5 \%$ of the mean response rate of last six sessions (cf. Schoenfeld, Cumming \& Hearst, 1956) and (b) when no systematic downward trend in mean response rates was observed during the last six sessions.

Response elimination. This phase lasted for 25 sessions. A DRO schedule of reinforcement was in effect in each component such that reinforcers were delivered after variable interreinforcer intervals (IRIs) without a key-peck response. Each key peck restarted the IRI. As described for the Training phase, reinforcers not delivered in either component were carried over to the next presentation of the same component.

During the initial 10 sessions of this phase, a DRO 30-s schedule was in effect in each component to reduce key pecking (and thus, increase pausing; cf. Lattal \& Boyer, 1980) by keeping the programmed reinforcement rate in each component equal to that programmed in the Training phase (two reinforcers per minute). During the terminal 15 sessions of this phase, for one component (hereafter, the lean component) the DRO was $60 \mathrm{~s}$ and for the other (hereafter, the rich component), it was $20 \mathrm{~s}$. Relative to the Training phase and the initial 10 sessions of this 
phase, the programmed reinforcement rates were one and three reinforcers per minute in the lean and rich components, respectively.

Resurgence. Reinforcers were discontinued (i.e., extinction was in effect) in both components of the multiple schedule. This condition lasted for 10 sessions. Other details were as described for the Training and Response-Elimination phases.

\section{Results and Discussion}

The number of sessions during the Training phase for each pigeon in this and in the subsequent experiments is in the rightmost column of Table 2. Figure 1 shows, for each pigeon, response (left graphs) and reinforcement rates (right graphs) in each schedule component during the last six sessions of the Training phase, and all sessions of the Response-Elimination and Resurgence phases. For each pigeon, reinforcement rates were stable, approaching the programmed rate of two reinforcers per minute in each schedule component during the last sessions of the Training phase. Additionally, response rates were higher in one component (the one later correlated with the lean component) during this phase for Pigeons 617, 681 and 627. During the initial sessions of the Response-Elimination phase, response rates decreased, and reinforcement rates increased in both components, for each pigeon. After 10 sessions, reinforcement rates were similar to those obtained during the Training phase for Pigeons 617, 627 and 580. Although for Pigeon 681 reinforcement rates were relatively low in both components in the initial sessions of the Response-Elimination phase due to consistent key pecking, responding was reduced relative to the Training phase. During the terminal sessions of the Response-Elimination phase, rates of key pecking were zero or near zero for each pigeon (response rates increased slightly in the lean component for Pigeon 617 during the last six 
sessions of this phase) and reinforcement rates in the lean and rich components were approximately one and three reinforcers per minute, respectively.

Resurgence of previously reinforced key pecking occurred for each pigeon in the Resurgence phase and its amount was related to the rate of reinforcement of alternative responding during the terminal sessions of the Response-Elimination phase. Rates of key pecking in each component increased relative to the terminal sessions of the ResponseElimination phase and, during some sessions, this increase was differential. For Pigeons 617, 627 and 580, higher rates of key pecking occurred in the lean component during the initial sessions of the Resurgence phase (for Pigeon 580, only during Session 2 of this this phase did more responding occurred in the rich than in the lean component). For Pigeon 681, this pattern of differential resurgence was observed across sessions. For the remaining sessions of this phase, resurgence was not differential between components for Pigeons 617, 627 and 580.

Figure 2 shows the frequency of key pecking in the rich and lean components during the last six sessions of the Response-Elimination phase, and all sessions of the Resurgence phase, for each pigeon. In addition to replicating the pattern of results shown in Figure 1, the data in Figure 2 show that, in some sessions of the Resurgence phase, responding was slightly higher in the rich than in the lean component (e.g., Pigeon 580 in Session 2; Pigeon 627 in Session 10; see especially Pigeon 617 from Sessions 3 through 10). In sessions in which more resurgence occurred in the rich component, however, differences in responding between components were lower than in sessions in which more resurgence occurred in the lean component. In general, resurgence was inversely related to the rate of reinforcement of alternative responding. These results are similar to those reported by Reed and Clark (2011) with humans. 
The present experiment extends the within-subject analyses conducted by Doughty et al. (2007), da Silva et al. (2008; Experiment 3) and Podlesnik and Shahan (2009, 2010) to the effects of reinforcement rate of alternative responding on resurgence. One interpretation of the present results is that alternative responding established by the DRO schedules was relatively more resistant to change in the component correlated with higher, rather than lower, reinforcement rates during the Response-Elimination phase (cf. Nevin \& Grace, 2000) and it occurred for longer periods in each schedule component during the Resurgence phase. Thus lower rates of key pecking would be predicted in the previously rich component. Although plausible, this account is difficult to assess independently because alternative responding was not directly measured, but defined generally as pausing. An interpretation of results based on the prevention-of-extinction hypothesis (Leitenberg et al., 1975), although also based on a form of response competition, suggests that resurgence occurs because differentially reinforcing alternative responding prevents previously reinforced responding of being extinguished.

The results of the present experiment are not consistent with those reported by Leitenberg et al., (1975; Experiment 3) and with the prevention-of-extinction hypothesis (see also Winterbauer \& Bouton, 2010). According to this account, the higher the frequency of Trainingphase responding during the Response-Elimination phase, the higher the probability of this responding being extinguished and the lower the probability of resurgence. Figure 3 shows the total number of key pecks (on a logarithmic scale), for each pigeon, during all sessions of the Response-Elimination and Resurgence phases. For Pigeons 617, 627 and 681, more responding occurred in the lean component in both Response-Elimination and Resurgence phases, results that do not support the prevention-of-extinction hypothesis. For Pigeon 580, although the number of key pecks was slightly higher in the rich component during the Response-Elimination 
phase, more responding occurred in the lean component during the Resurgence phase. One factor that could be responsible for this difference in results is that Leitenberg et al. used a concurrent extinction VI, instead of a DRO, schedule to reduce the frequency of previously reinforced responding and to reinforce alternative responding (see Experiment 6, below). Even though in Leitenberg et al.'s study the differences in resurgence between groups were statistically significant, data were presented as group means and no measures of variability were provided. In addition, the obtained reinforcement rates of alternative responding were not reported and it is not clear how that might have affected their results.

The present results also did not replicate those of Winterbauer and Bouton (2010; Experiments 1 and 2). These authors used a procedure in the Response-Elimination phase similar to that arranged by Leitenberg et al. (1975, Experiment 3), but reported no differences in resurgence as a function of different reinforcement rates of alternative responding (thus, also describing results which do not support the prevention-of-extinction hypothesis). As in Leitenberg et al.'s study, their results are presented as group means, obfuscating variability and individual differences in responding.

The differential resurgence observed in the initial sessions of the Resurgence phase is in contrast to the predictions of Shahan and Sweeney's (2011) model. Although their model always predicts mode resurgence in the rich than in the lean component, considering the schedule parameters used in the present experiment (VI 30-s schedules in the Training phase, and DRO 20-s and DRO 60-s schedules in the Response-Elimination phase) it also predicts that the difference in resurgence between components should be smaller than when leaner schedules are in effect during the Response-Elimination phase (e.g., a VI 240 s in each component; see Shahan \& Sweeney, Figure 4, p. 100; see Appendix A and Figures A1 and A2; see also Appendix B for 
an analysis of the results of the present experiment based on this model). Data from sessions in which resurgence was not differential or in which differences between components were not extreme (see Figure 2), suggest that the parameters of the schedules might be relevant to account for the present results.

Both the order in which schedule components are presented during Resurgence-phase sessions and differences in response rates between components during the Training phase have been suggested to affect resurgence (da Silva et al., 2008; Reed \& Morgan, 2007). Thus, they are relevant as well to the present discussion. Da Silva et al. (p. 325) suggested that the use of multiple schedules might bias the results in studies of differential resurgence. Because multipleschedule components must alternate, and one of them must be presented first in a condition, or in a session, more resurgence might occur in one component just because it was presented first. To assess if this occurred in the present experiment, Figure 4 shows, for each pigeon, the mean frequency of key pecking in the lean and rich components as a function of within-session order of component presentation. Mean frequencies are shown for Resurgence-phase sessions in which the first component was lean (left graphs) or rich (right graphs). Table 3 shows, for each pigeon in this and the following experiments, the first component in each Resurgence-phase session and its rightmost column shows the frequency of sessions starting with the rich and lean components, respectively.

Figure 4 shows that the frequency of key pecking in each component was not related to either component being presented first in a session or condition (see also Table 3). Even Pigeon 580, which responded more in the lean component when it was presented first, also responded more in the lean component (or with no differences between components) when the rich component was the presented first. Thus, order of component presentation cannot account for 
the general pattern of more resurgence in the lean than in the rich component in this experiment. These results were observed even though the rich component was presented first in the Resurgence phase, for Pigeons 617, 681 and 580, and in six of 10 sessions in the Resurgence phase, for Pigeon 618 (see Table 3).

Additionally, da Silva et al. (2008; see also Reed \& Morgan, 2007; but see Podlesnik \& Shahan, 2009, 2010 for an alternative account) suggested that more resurgence occurs when response rates during the Training phase are high rather than low. For three pigeons (see Figure 1) higher rates of responding occurred during the Training phase in the component that was later the lean component. Even though each pigeon's rate of responding was near zero in each component during the terminal sessions of the Response-Elimination phase (and reinforcement rates approximated the programmed rates), these differences in rate of responding cannot be eliminated as a source of control in the present experiment. Due to the pigeons' extensive experimental histories, this limitation was addressed by replicating the present experiment with experimentally-naïve pigeons, and by assessing Training-phase response rates in each component to define the rich and lean components during the Response-Elimination phase.

\section{Experiment 1B}

\section{Method}

\section{Subjects and Apparatus}

Four experimentally-naïve male White Carneau pigeons were maintained as described in Experiment 1A. The apparatus and stimulus conditions in effect in each phase were the same as those used in Experiment 1A (see Table 2 for a description of the keylight colors used for each pigeon). 


\section{Procedure}

Pretraining. Each pigeon was exposed initially to magazine training. After each ate reliably from the hopper when it was raised, key pecking was established by differential reinforcement of successive approximations. A multiple FR 1 FR 1 was then in effect such that key pecking produced a reinforcer and changed the keylight color in effect until 60 reinforcers were delivered. When key pecking occurred reliably in the presence of each keylight color, the FR requirements were increased in steps of one or two responses over 2-3 sessions, and until a FR 5 was in effect in each component for 3-5 sessions. Subsequently, each pigeon was exposed to a multiple VI VI schedule as described in Experiment 1A. The values of each VI were increased to $30 \mathrm{~s}$ across sessions and the duration of each component was increased accordingly to $180 \mathrm{~s}$. That is, each pigeon was exposed to $2-5$ sessions in which the parameters of the VI schedules were, $5 \mathrm{~s}, 10 \mathrm{~s}, 15 \mathrm{~s}$, and $20 \mathrm{~s}$ and component duration was, respectively, $30 \mathrm{~s}, 60 \mathrm{~s}, 90$ $\mathrm{s}$, and $120 \mathrm{~s}$.

Training, Response-Elimination and Resurgence phases. Each phase was conducted as described in Experiment 1A. The components with higher and lower response rates during the Training phase were set as the rich and lean components, respectively, during the terminal sessions of the Response-Elimination phase.

\section{Results and Discussion}

The Training phase was in effect for 20 sessions for Pigeons 56, 1, 60 and 873. Figure 5 shows response and reinforcement rates in each schedule component during the last six sessions of the Training phase, and all sessions of the Response-Elimination and Resurgence phases. For each pigeon, reinforcement rates in the Training phase approximated the programmed rate of two reinforcers per minute in each component and similar and stable response rates in each 
component were observed for Pigeons 56 and 60. Responding in each component was stable for Pigeons 1 and 873 in this phase, although slight differences in rate of responding between components were observed. In general, the differences in rate of responding between components found in Experiment 1A (see Figure 1) were not replicated.

During the initial sessions of the Response-Elimination phase, rate of responding decreased, and reinforcement rates increased, in each component for Pigeons 1 and 60. Although responding was near zero for Pigeons 56 and 873 after the initial sessions of this phase, due to low rates of responding reinforcement rates were less than two reinforcers per minute in each component. During the terminal sessions of the Response-Elimination phase, rate of responding in each component was near zero, for each pigeon, and reinforcement rates for Pigeons 1 and 60 were approximately one and three reinforcers per minute in the lean and rich components, respectively. Reinforcement rates in these sessions were less controlled for Pigeons 56 and 873 due to low, but consistent, rates of responding - although reinforcement rates were generally higher in the rich then in the lean component for these pigeons. Resurgence occurred only for two pigeons. For Pigeon 1 responding was slightly higher in the rich than in the lean component during the Resurgence-phase sessions and for Pigeon 60, differences in rate of responding between components were not systematic. Resurgence of key pecking was not observed for Pigeons 56 and 873.

These results also can be seen in Figure 6, which shows the frequency of key pecking in each component during the last six sessions of the Response-Elimination phase and all sessions of the Resurgence phase, for each pigeon. Although responding was near zero for Pigeons 1 and 60 during the Response-Elimination phase (and resurgence was observed, as previously described), key pecking occurred consistently and with a low frequency in both components 
during these sessions for Pigeons 56 and 873. For these two pigeons, key pecking during the Resurgence phase occurred with a lower frequency than during the Response-Elimination phase and, thus, not characterizing resurgence in either component. For this reason, the following analyses were conducted with data from Pigeons 1 and 60.

Figure 7 shows the total number of key pecks (on a logarithmic scale) in each component for Pigeons 1 and 60 during all sessions of the Response-Elimination and Resurgence phases. Although slightly more key pecking occurred for each pigeon in the rich than the lean component during the Resurgence phase (with differences between components being more pronounced for Pigeon 1), the number of key pecks was similar between components during the Response-Elimination phase. For this reason it is difficult to say that, in either component, reinforcers for alternative responding prevented the extinction of key pecking. As in Experiment 1A (see Figure 3), these results do not support the prevention-of-extinction hypothesis (Leitenberg et al., 1975).

The resurgence observed for Pigeons 1 and 60 was not affected by the order of component presentation during Resurgence-phase sessions. Figure 8 shows, for these pigeons, the mean frequency of key pecking in the lean and rich components as a function of withinsession order of component presentation and the first component in a session (see Table 3 for the first component in each Resurgence-phase session, for each pigeon). Independent of rich and lean components being presented first in a session, mean frequency of key pecking was generally higher in the rich component for Pigeon 1. For Pigeon 60, when the lean component was presented first (left graph), more responding occurred in this component during the first, second and third presentations, but responding also increased in the rich component across presentations and, overall, no systematic differences between components were observed (see also Figure 5). 
When the rich component was presented first (right graph), more responding occurred in the lean component for Pigeon 60 during the first and second presentations of this component, but mean responding was generally higher in the rich component. Similar to Experiment 1A, no systematic relation between order of component presentation and responding in each component was observed. (Similar analyses of Resurgence-phase responding in terms of total number of key pecks [Figures 3 and 7], and of responding as a function of order of component presentation [Figures 4 and 8], were conducted for each experiment in the present study and yielded similar results to those in Experiments 1A and 1B. For this reason, except for an analysis of total number of key pecks in Experiment 3 [see below] these analyses were not reported in the subsequent experiments in the present study).

The differential resurgence observed in Experiment 1A was not replicated when differences between components in Training-phase response rates were reduced in the present experiment (see Figure 5). Given that schedules and schedule parameters were similar in these experiments, the differences in response rates between components during the Training phase likely affected the results of Experiment 1A (e.g., da Silva et al. 2008; Reed \& Morgan, 2007). In addition, the interpretation of differential resurgence in terms of relative resistance to change of alternative responding (Nevin \& Grace, 2000) described in that experiment cannot account for the results obtained with Pigeons 1 and 60.

Resurgence occurred in the present experiment with only two pigeons, and it was differential only for Pigeon 1. More resurgence occurred in the rich than the lean component for this pigeon, but differences in responding between components were not extreme (a pattern also observed during some Resurgence-phase sessions in Experiment 1A; see Figures 1 and 2). Considering the schedule parameters used in the present experiment, the results for this pigeon 
are in the direction predicted by Shahan and Sweeney's (2011) model. The results of Pigeon 60 were similar to those reported by Winterbauer and Bouton (2010; Experiments 1 and 2), as no systematic relation between responding in the Resurgence phase and reinforcement rate of alternative responding was observed. In addition, when differences in responding between components occurred in the Resurgence phase, they were not extreme. The results for this pigeon also are similar to those reported by Mulick et al. (1976), in that, at least for some squirrel monkeys in their study, no systematic differences in resurgence occurred between multipleschedule components. Although Mulick et al. did not manipulate rate of reinforcement of alternative responding, the schedule parameters they used during the Response-Elimination phase were similar to those used in the present experiment (e.g., DRO $20 \mathrm{~s}$ or $30 \mathrm{~s}$ ).

Finally, exposing the pigeons to initial sessions of DRO-reinforcement (i.e., the initial sessions of the Response-Elimination phase) may have lengthened the Response-Elimination phase to the point that the probability that resurgence would occur was reduced (e.g., Cleland et al., 2000; Leitenberg et al., 1975; Experiment 4; but see Lieving \& Lattal, 2003, Experiment 1). In addition, in the DRO schedules in this experiment, and also in Experiments 2 through 4, alternative responding was defined generally as "pausing, or not pecking" but no attempt was made to control what the pigeons did in each schedule component. These aspects of the procedure were addressed in Experiment 5 and, particularly, Experiment 6 (see description of each experiment below).

Based on the results of Experiments 1A and 1B, the effects on resurgence of schedule parameters in effect during Training and Response-Elimination phases were studied in Experiment 2. That is, during the Response-Elimination phase, rates of reinforcement in one 
schedule component were similar to those in the Training phase, and in the other, reinforcement rates were higher or lower than those programmed in that phase.

\section{Experiment 2}

This experiment was conducted to assess resurgence when reinforcement rates during the terminal sessions of the Response-Elimination phase were equal, in one multiple-schedule component, and lower or higher in the other, than the programmed reinforcement rate in each component during the Training phase.

\section{Method}

\section{Subjects and Apparatus}

Four male White Carneau pigeons with previous exposure to various schedules of foodreinforcement were maintained as in Experiment 1. The apparatus and stimulus conditions in effect in each phase were as described in Experiment 1 (see Table 2 for the keylight colors used for each pigeon).

\section{Procedure}

The Training and Resurgence phases were as described in Experiment 1. Due to the pigeons' experimental histories, a Pretraining phase as conducted in Experiment 1B was not necessary. The Response-Elimination phase lasted for 25 sessions for Pigeons 691, 847 and 775 but, due to a programming error, it lasted for 27 sessions for Pigeon 806 . The schedules and schedule parameters in effect during the Response-Elimination phase are shown in Table 1. During the initial 10 sessions of this phase, DRO 30-s schedules were in effect such that the programmed reinforcement rates were equal to those programmed in the Training phase in each component of the multiple schedule. 
During the terminal 15 sessions of the Response-Elimination phase, two pigeons (691 and 847) first were exposed to a multiple DRO $30 \mathrm{~s}$ (lean) DRO $20 \mathrm{~s}$ (rich) schedule, and the other two pigeons (806 and 775) were exposed to a multiple DRO $30 \mathrm{~s}$ (rich) DRO $60 \mathrm{~s} \mathrm{(lean)} \mathrm{-}$ Pigeon 806 was exposed to two additional sessions during which this schedule was in effect.

Each pigeon then was exposed to a replication of each phase in which the Training, Response-Elimination and Resurgence phases lasted for 20,25 and 10 sessions, respectively. In the terminal sessions of the Response-Elimination phase, the reinforcement rates were reversed for each pigeon such that the component that was rich (or lean) during the first exposure was the lean (or rich) during the replication. In addition, to make the difference in reinforcement rates between components more extreme, in the terminal sessions of the Response-Elimination phase a DRO $15 \mathrm{~s}$ and a DRO $90 \mathrm{~s}$ were used (instead of a DRO $20 \mathrm{~s}$ and DRO $60 \mathrm{~s}$ ). For example, pigeons that were exposed first to a multiple DRO 30 s DRO 20 s (Pigeons 691 and 847) or to a multiple DRO 30 s DRO 60 s (Pigeons 806 and 775) were exposed to, respectively, a multiple DRO $30 \mathrm{~s}$ DRO $90 \mathrm{~s}$ and a multiple DRO $30 \mathrm{~s}$ DRO $15 \mathrm{~s}$ during the replication. For each pigeon, the stimulus correlated with the DRO 30-s during the terminal sessions of the ResponseElimination phase was the same during the first exposure and replication (see Table 2).

\section{Results and Discussion}

The Training phase during the first exposure lasted for 20 sessions for Pigeons 691, 847 and 775, and for 25 sessions for Pigeon 806. Figure 9 shows response and reinforcement rates in each component for the last six sessions of the Training phase, and all sessions of the ResponseElimination and Resurgence phases during the first exposure. Response rates were stable and similar between components during the Training phase for each pigeon and small differences between components can be seen only for Pigeon 806 . 
During the initial sessions of the Response-Elimination phase, rate of responding was reduced in each component and reinforcement rates increased accordingly, approaching the programmed rate of two reinforcers per minute for Pigeons 691 and 806 (for this pigeon, rate of responding was consistently reduced relative to the Training phase in the first session of the Response-Elimination phase). For Pigeons 847 and 775, reinforcement rates were lower than the programmed rate due to low rates of key pecking in both components. After 10 sessions, rate of responding was near zero in each component for Pigeons 691, 806 and 847, but key pecking still occurred consistently in both components (although at low rates) for Pigeon 775.

For Pigeons 806 and 847, reinforcement rates approximated the programmed rates (respectively of two and one, and three and two reinforcers per minute in the rich and lean components), and response rates in both components were zero or near zero during the terminal sessions of the Response-Elimination phase. The same control over reinforcement rates was not obtained for Pigeon 691, and especially for Pigeon 775, due to consistent key pecking in both components (even though at low rates) during these sessions - although during the last six sessions of the Response-Elimination phase, reinforcement rates approximated the programmed rates for Pigeon 691.

During the Resurgence phase, a slight increase in rate of responding relative to the terminal sessions of the Response-Elimination phase occurred only for Pigeon 847 and resurgence was not observed for the other pigeons. Due to the $\mathrm{Y}$-axis scales in response-rate graphs in Figure 9, this can be seen more clearly in Figure 10, which shows key-pecking frequency in each component during the last six sessions of the Response-Elimination phase and all sessions of the Resurgence phase during the first exposure. For Pigeon 847 differences in responding between components were not related to the rate of reinforcement in effect during the 
terminal sessions of the Response-Elimination phase (i.e., in Session 2, for example, more responding occurred in the lean than in the rich component whereas in Session 4, the opposite was observed), nor by which component was presented first in these sessions (in both Sessions 2 and 4, the lean component was presented first; see Table 3). Pigeon 806 responded equally in each component and frequencies were extremely low. Pigeon 691 had a similar pattern of low frequencies during Resurgence-phase sessions, and responding occurred in the rich component at levels observed during the Response-Elimination phase. Finally, Pigeon 775 responded similarly in each component across Resurgence-phase sessions and frequencies were similar to those observed for this pigeon during the Response-Elimination phase.

The results of the replication of each phase are presented in Figures 11 and 12 which show, respectively, response and reinforcement rates in each component, during Training, Response-Elimination and Resurgence phases, and the frequency of key pecking in each component during Response-Elimination and Resurgence phases. For each pigeon, the results were similar during the Training and the initial sessions of the Response-Elimination phase for the first exposure and the replication. As can be seen in Figure 11, rate of responding was stable and similar between schedule components during the Training phase. Rate of responding decreased to zero or near zero in each component during the initial sessions of the ResponseElimination phase for Pigeons 691, 806 and 847 - for Pigeon 775, the same pattern of low rates of responding during these sessions reduced the obtained reinforcement rates in each component, which were still lower than the programmed rate after 10 sessions.

During the terminal sessions of the Response-Elimination phase a greater difference in reinforcement rates between components was programmed relative to the first exposure. For each pigeon, the rate of reinforcement during these sessions approximated the programmed rate - 
even for Pigeon 775, which responded consistently in each component, although at low rates. In the Resurgence-phase, rate of responding increased slightly relative to the Response-Elimination phase for Pigeons 806 and 847 and no resurgence occurred for the other two pigeons, as during the first exposure to each phase (see Figures 9 and 10). Pigeons 691 and 775 responded in each component during the Resurgence-phase sessions as during the terminal sessions of the Response-Elimination phase.

The resurgence observed for Pigeons 806 and 847, as can be seen in Figure 12, was similar to that obtained for Pigeon 847 during the first exposure. That is, in some sessions more responding occurred in the rich than in the lean component (e.g., Sessions 2 and 4 of the Resurgence phase for Pigeons 806 and 847, respectively) and, in others, the opposite was observed (Sessions 4 and 5 of this phase for Pigeons 806 and 847, respectively). Additionally, in some sessions resurgence occurred in both components and was not differential, for either pigeon (e.g., Sessions 1 and 2 for Pigeons 806 and 847, respectively). These results, as can be seen in Table 3, have no systematic relation with which schedule component was presented first in a session during the Resurgence phase.

The results of the first exposure and of the replication are similar to those of Experiment 1B in that no systematic relation between rate of reinforcement of alternative responding and resurgence was observed. When differences in responding between rich and lean components occurred in each Resurgence phase in the present experiment, these differences were not extreme (as occurred for Pigeons 1 and 60 in Experiment 1B). When resurgence occurred (in three of eight cases in this experiment, i.e., for Pigeon 847 during the first exposure and replication, and for Pigeon 806, during the replication), in some sessions more responding occurred in the rich 
component, and in others, in the lean component; still in other sessions, resurgence was not differential.

In summary, the results of this experiment are similar to those of Winterbauer and Bouton (2010; see also Mulick et al., 1976) in that no systematic differences in resurgence occurred as a function of differences in reinforcement rates of alternative responding. In addition, these results further question the results of Experiment $1 \mathrm{~A}$ and their interpretation in terms of relative resistance to change of alternative responding, described in that experiment.

Although the present results were not confounded by differences in response rates between schedule components during the Training phase (cf., da Silva et al., 2008; see Experiment 1, above), little to no resurgence occurred in the present experiment. As in Experiment 1, the DRO schedules and the schedule parameters in effect across ResponseElimination phases were used to increase the probability of obtaining resurgence (cf. Doughty et al., 2007). The reasons for not observing more resurgence in both components in the present experiment, as well as in Experiment 1B, are not clear. The exposure to the DRO schedules per $s e$ is not an explanation, because resurgence has been observed commonly and consistently when these schedules were in effect in the Response-Elimination phase (e.g., Cançado \& Lattal, 2011, Experiment 2; da Silva et al.; Doughty et al.; see also the present Experiment 6, below), even after 15 to 30 sessions of exposure to the Response-Elimination phase (e.g., da Silva et al.; Doughty et al.; but see Leitenberg et al., 1975, Experiment 4). Thus, the schedules, the schedule parameters (which differed between the first exposure and replication in the present experiment) and the length of the Response-Elimination phase individually or in concert with one another might have affected the results of Experiments $1 \mathrm{~B}$ and 2, reducing the probability that resurgence occurred. 


\section{Experiment 3}

This experiment was conducted to assess resurgence when key pecking was extinguished before differential reinforcement rates of alternative responding were in effect during the Response-Elimination phase (cf. Epstein, 1983; see also Lieving \& Lattal, 2003; and Winterbauer \& Bouton, 2011). The procedure was a replication of Experiments 1 and 2 in which a within-subject test of the prevention-of-extinction-hypothesis of resurgence was conducted (cf., Leitenberg et al., 1975; Cleland, et al., 2000; Cleland, et al., 2001; but see Winterbauer \& Bouton, 2010).

\section{Method}

\section{Subjects and Apparatus}

The three pigeons used in Experiment 1A (617, 627 and 681) and another female White Carneau pigeon (874), with previous exposure to various schedules of food-reinforcement, served. Each pigeon was maintained as described in Experiment 1, and the apparatus and stimulus conditions were as described in that experiment (see Table 2 for the keylight colors used for each pigeon).

\section{Procedure}

The Training and Resurgence phases were conducted as described in Experiment 1. The Response-Elimination phase lasted 25 sessions for each pigeon. Before a multiple DRO $20 \mathrm{~s}$ DRO $60 \mathrm{~s}$ was in effect during the terminal 15 sessions of this phase, reinforcers were discontinued in both components of the multiple schedule for 10 sessions (i.e., extinction was in effect during the initial sessions of this phase; see Table 1). As in Experiment 1B, the rich and lean components in the Response-Elimination phase were those with higher and lower response 
rates during the Training phase, respectively. Other details of the procedure were as described in Experiment 1.

\section{Results and Discussion}

The Training phase lasted for 20 sessions for Pigeons 617, 627 and 681, and for 25 sessions for Pigeon 874. Figure 13 shows response and reinforcement rates in each component during the Training, Response-Elimination and Resurgence phases. During the last six sessions of the Training phase, differences in rate of responding between components were observed only for Pigeon 627. For each pigeon, reinforcement rates in each component during this phase approximated the programmed rate of two reinforcers per minute. Rate of responding decreased in each component when reinforcers were discontinued during the initial sessions of the Response-Elimination phase. After 10 sessions, rate of responding was near zero in both components for Pigeons 627 and 681. Similar results were observed for Pigeon 617, but rates of responding were slightly higher in one (later the lean) component during these sessions. Responding in both components was reduced relative to the Training phase for Pigeon 874, but was higher than observed for the other three pigeons during these sessions.

When the multiple DRO 20-s DRO 60-s schedule was in effect, rate of responding increased in both components and was higher in the rich than the lean component for Pigeons 617, 874 and 627. This effect (which could be described as response-induction; i.e., the recovery of previously reinforced responding when response-dependent reinforcers are effected; e.g., Reynolds, 1964; and response-reinstatement, i.e., the recovery of previously reinforced responding when response-independent reinforcers are effected; e.g., Franks \& Lattal, 1976; because under DRO schedules reinforcer deliveries were dependent on not pecking, but were delivered if the pigeons emitted any other responses during the IRI) was observed only initially 
during the terminal sessions of the Response-Elimination phase for Pigeons 874 and 627, but occurred for more sessions for Pigeons 617 and 681 (see, for example, the increase in rate of responding in the lean component for Pigeon 617 later during this phase).

The results obtained in the transition from the initial to the terminal sessions of the Response-Elimination phase are similar to those of Winterbauer and Bouton (2011; Experiment 2). Previously reinforced, but subsequently extinguished, lever pressing by rats recurred when both response-dependent and -independent reinforcers were in effect during the ResponseElimination phase, when an alternative lever-press response could be emitted. They suggested, that response-induction (e.g., Reynolds, 1964) or reinstatement (e.g., Franks \& Lattal, 1976) might be involved in response recovery when Training-phase responding is extinguished before alternative responding is reinforced, as conducted, for example, by Bruzek et al. (2009), by Epstein (1983), and in the present experiment. For each pigeon, rate of responding was zero or near zero (e.g., Pigeon 617) after the terminal sessions of the Response-Elimination phase, and reinforcement rates approximated the programmed rate in each component. The data in Figure 13 show that when reinforcers were discontinued in the Resurgence phase, a slight increase in rate of responding relative to the last six sessions of the Response-Elimination phase was observed in the lean component only for Pigeon 681 and resurgence did not occur for the other three pigeons.

Figure 14 shows the results as frequencies of key pecking in each component during the last six sessions of the Response-Elimination phase, and all sessions of the Resurgence phase. Resurgence would be described in the lean component only for Pigeon 681, during Session 3 of the Resurgence phase. This increase in responding relative to the Response-Elimination phase, however, was only slight. As for the other three pigeons, it could be argued that resurgence did 
not occur for Pigeon 681 and, consequently, that the present experiment was not a fair test of the prevention-of-extinction hypothesis (Leitenberg et al., 1975; Rawson et al., 1977).

The main assumption in the prevention-of-extinction hypothesis (Cleland et al., 2000; Leitenberg et al., 1975) is that reinforcing alternative responding prevents the extinction of previously reinforced responding. Thus, the occurrence of previously reinforced responding in the Resurgence-phase would be inversely related to its occurrence during the ResponseElimination phase. As in Experiment 1, Figure 15 shows an analysis of total number of responses (on logarithmic scale) in each component during all sessions of the ResponseElimination and Resurgence phases. The data in this figure show that this inverse relation between responding in Response-Elimination and Resurgence phases was not observed, thus, not supporting the prevention-of-extinction account. Although for Pigeon 874 this inverse relation seems to have occurred, differences in responding between rich and lean components across the two phases were negligible (more responding in the rich than in the lean component was likely affected by this pigeon's responding during the initial 10 sessions of the Response-Elimination phase and during the first session of exposure to the multiple DRO $20 \mathrm{~s}$ DRO $60 \mathrm{~s}$ (see Figures 13 and 14).

In summary, the results of this experiment were similar to those of Experiments $1 \mathrm{~B}$ and 2 in that responding during the Resurgence-phase sessions was not a function of reinforcement rate of alternative responding during the Response-Elimination phase. Also as in Experiments 1 and 2 , the results of the present experiment were not affected by the order of component presentation during each Resurgence-phase session (see Table 3). Exposure to extinction during the initial sessions of the Response-Elimination phase per se seems unlikely to account for the results of the present experiment because (a) an absence of resurgence, and resurgence that was not 
differential during some Resurgence-phase sessions also were obtained in Experiments $1 \mathrm{~B}$ and 2 when DRO schedules were in effect during the Response-Elimination phase; and (b) resurgence has been reported when Training-phase responding is extinguished before reinforcing alternative responses (e.g., Epstein, 1983; Lieving \& Lattal, 2003; Winterbauer \& Bouton, 2011). Maybe, resurgence would have been observed in the present experiment if the pigeons were exposed to fewer sessions during the Response-Elimination phase, regardless the schedules of reinforcement in effect (cf. Cleland et al., 2000; see Experiments 5 and 6, below).

Another possibility is that arranging differential reinforcement rates of alternative responding during the initial sessions of the Response-Elimination phase - and equal reinforcement rates during the terminal sessions of this phase - would produce differential resurgence. As sequential exposures to different reinforcement conditions were in effect during the Response-Elimination phase in Experiments 1B through 3, responding during the Resurgence phase could have been affected by the order in which the pigeons were exposed to these conditions. Such order effects were studied in Experiment 4.

\section{Experiment 4}

The order of exposure to conditions during the Training phase has been shown to affect resurgence. When response sequences across two or more operanda are trained sequentially (e.g., first, Sequence 1 is reinforced, then only Sequence 2 is reinforced, and so on), resurgence of responding trained first, or primacy effects, and last, or recency effects, have been reported (see, for example, Reed \& Morgan, 2006, for a study with rats; and Mechner \& Jones, 2011, for studies with humans; see also Bruzek et al., 2009, Experiment 2). The present experiment was conducted to assess whether the order of exposure to conditions arranging different reinforcement rates of alternative responding during the Response-Elimination phase would 
affect resurgence. Specifically, pigeons were exposed to the same schedules of reinforcement and schedule parameters in effect during the initial and terminal sessions of the ResponseElimination phase of Experiment 1, but in reverse order relative to that experiment.

\section{Method}

\section{Subjects and Apparatus}

Four experimentally-naïve male White Carneau pigeons were maintained as described in Experiment 1. The apparatus and the stimulus conditions were as in Experiment 1 (see Table 2 for the keylight colors used for each pigeon).

\section{Procedure}

For each pigeon, the Pretraining, Training, and Resurgence phases were as described in Experiment 1B. The Response-Elimination phase lasted for 25 sessions for Pigeons 12, 2 and 72 and, due to a programming error, for 31 sessions for Pigeon 828. During this phase, each pigeon was exposed to a multiple DRO 20-s DRO 60-s schedule for the initial 15 sessions - the rich and lean components during these sessions were those in which higher and lower response rates occurred during the Training phase, respectively. Then, a DRO $30 \mathrm{~s}$ was in effect in each schedule component for the terminal 10 sessions of this phase, for Pigeons 12, 2 and 72, and for the terminal 15 sessions, for Pigeon 828 .

After being exposed to the multiple DRO 30 s DRO 30 s for seven sessions, Pigeon 828 was inadvertently exposed to one session in which a multiple VI $30 \mathrm{~s}$ VI $30 \mathrm{~s}$, similar to that programmed during the Training phase, was in effect. Three sessions were then conducted (totaling 10 sessions) in which the DRO $30 \mathrm{~s}$ was again in effect in each schedule component. To further reduce key pecking and expose this pigeon to the programmed reinforcement rates in 
each component before the Resurgence phase, five additional sessions were conducted in which the multiple DRO $30 \mathrm{~s}$ DRO $30 \mathrm{~s}$ was in effect.

Thus, differences between components in programmed reinforcement rates were in effect to reduce key pecking while reinforcing alternative responding (i.e., a multiple DRO 20 s DRO $60 \mathrm{~s}$ ), but maintenance of these low-to-zero rates of responding was conducted by programming reinforcement rates similar to those in the Training phase (i.e., a multiple DRO $30 \mathrm{~s}$ DRO $30 \mathrm{~s}$ ).

\section{Results and Discussion}

The Training phase lasted for 20 sessions for Pigeons 12, 2 and 72, and for 25 sessions for Pigeon 828. Response and reinforcement rates in each schedule component during the Training, Response-Elimination and Resurgence phases are shown for each pigeon in Figure 16. For each pigeon, response rates in each component were stable during the last six sessions of the Training phase and slight differences in responding between components were observed for Pigeons 12, 2 and 72 during these sessions. For each pigeon, reinforcement rates were approximately two reinforcers per minute during these sessions.

In the initial sessions of the Response-Elimination phase, for each pigeon, rate of responding in both components was reduced relative to the Training phase. For Pigeons 12 and 72, response rates were near zero in each component, and reinforcement rates approximated one and three reinforcers per minute, respectively, in the rich and lean components. Due to low-rate responding in both components, the same control over reinforcement rates was not obtained for Pigeons 2 and 828, although in these sessions reinforcement rates generally were higher in the rich than in the lean component. Response rates were near zero, and reinforcement rates approximated the programmed rate in each component, for Pigeons 12, 2 and 72, during the terminal sessions of the Response-Elimination phase. Similar results were obtained for Pigeon 
828 during the first seven sessions of exposure to the multiple DRO $30 \mathrm{~s}$ DRO $30 \mathrm{~s}$. In the session in which VI $30 \mathrm{~s}$ schedules were in effect in each component, rate of responding increased, and reinforcement rates in each component were practically unaltered relative to the previous session. When the DRO 30-s schedules were reinstated for Pigeon 828, responding decreased to near zero, and reinforcement rates approximated two reinforcers per minute, in each component. For each pigeon, rate of responding did not change systematically in the transition from initial to terminal sessions of the Response-Elimination phase (as in Experiment 3).

Resurgence occurred in both components only for Pigeons 12 and 2. These results can be seen also in Figure 17, which shows the frequency of key pecking in each component during the last six sessions of the Response-Elimination phase and all sessions of the Resurgence phase. The slight increases in responding in both components during the Resurgence phase for Pigeons 12 and 2 were similar to those observed in Experiments 1B and 2. That is, in some sessions, more responding occurred in the lean component, in other sessions, in the rich component (e.g., respectively Sessions 8 and 9, for Pigeon 12, and, Sessions 6 and 8, for Pigeon 2). As noted in the previous experiments in the present study, in some Resurgence-phase sessions responding in both components was not differential. For Pigeons 72 and 828 resurgence did not occur, and no relation between responding in the Resurgence phase and reinforcement rates of alternative responding were observed for these pigeons. Additionally, Resurgence-phase responding for each pigeon was not related to which component was presented first in each session, as can be seen in Table 3, and also as occurred in the previous experiments in the present study.

Thus, independent of the order in which differential and non-differential reinforcement rates of alternative responding were in effect during the Response-Elimination phase, no relation between resurgence and reinforcement rates of alternative responding was observed. In contrast 
to the previously described effects of exposure to conditions during the Training phase on resurgence (i.e., primacy and recency effects; e.g., Reed \& Morgan, 2006) at least with the schedules, schedule parameters and number of sessions in each phase of the present experiment, the order of exposure to the conditions in effect during the Response-Elimination phase did not affect resurgence. It should be noted that different from studies in which primacy and recency effects have been observed, no aspect of responding was differential during the initial and terminal sessions of the Response-Elimination phase in the present experiment (e.g., as different response sequences were established sequentially in Reed \& Morgan's study; see also Mechner \& Jones, 2011). That is, differential rates of reinforcement were in effect during the ResponseElimination phase of the present experiment for not pecking.

In summary, similar results were obtained in Experiments 1B through 4 in that (a) little to no resurgence occurred in each component of the multiple schedule across Resurgence phases; (b) when resurgence occurred, no systematic relation with the differential reinforcement rates of alternative responding was observed, i.e., in some sessions, more resurgence occurred in the rich component, in other sessions, in the lean component, and was undifferentiated between components in other sessions (e.g., Winterbauer \& Bouton, 2010; Mulick et al., 1976). In addition, these results were not affected by the first component presented in Resurgence-phase sessions (cf. da Silva et al., 2008), neither by the order of component presentation within Resurgence-phase sessions (see Figures 4 and 8, respectively, in Experiments 1 and 2; and Table 3). The number of sessions conducted during the Response-Elimination phase, the DRO schedules and the parameters of these schedules in the initial and terminal sessions of this phase (cf. Shahan \& Sweeney, 2011; see also Mulick et al., 1976) were described as variables that 
could be responsible for the general pattern of results obtained in the present study. These variables were further assessed in the following two experiments.

In Experiments 5 and 6, the number of sessions in the Response-Elimination phase was reduced and different schedules of reinforcement were in effect during this phase, relative to Experiments 1 through 4. In Experiment 5 fixed-, instead of variable-, DRO schedules (cf. Doughty et al., 2007) were in effect in each multiple-schedule component during the ResponseElimination phase. In Experiment 6, the effects of reinforcement rates of alternative responding on resurgence were assessed when alternative responding consisted of pecking a different key.

\section{Experiment 5}

This experiment was conducted to assess if the results of Experiments 1 through 4 were affected by the periodicity of reinforcement arranged by the variable-DRO schedules during the Response-Elimination phase (e.g., Doughty et al., 2007, Experiment 3). Resurgence was assessed when reinforcement rates of alternative responding were arranged by fixed-DRO schedules. In addition, the number of sessions during each phase, particularly in the ResponseElimination phase, was reduced relative to the previous experiments in the present study.

\section{Method}

\section{Subjects and Apparatus}

Two male White Carneau pigeons (Pigeons 60 and 72, which served as subjects in Experiments 1B and 4, respectively) were maintained as described in Experiment 1. The apparatus and stimulus conditions were the same as used for each of these pigeons in Experiments 1B and 4 (see Table 2 for the keylight colors used for each pigeon). 


\section{Procedure}

The Training, Response-Elimination, and Resurgence phases lasted, respectively, 10, 15 and 10 sessions, for each pigeon. Each phase was conducted as described in Experiment 1B, with the exception that instead of variable, the IRIs were of fixed duration in each DRO schedule during the Response-Elimination phase. In addition, in the Response-Elimination phase, each pigeon was exposed directly to a multiple DRO $20 \mathrm{~s}$ DRO $60 \mathrm{~s}$ for 15 sessions (the IRIs in each schedule component were restarted only when key pecking occurred and after reinforcer deliveries). If differences in responding between components occurred during the Training phase, the rich and lean components during the Response-Elimination phase were those with higher and lower response rates, respectively, for each pigeon. For Pigeon 72, the rich and lean components during the Response-Elimination phase were correlated with red and amber keylights (as for this pigeon in Experiment 4). For Pigeon 60, rich and lean components were correlated with amber and red keylights, respectively, and thus, reversed in relation to the stimulus conditions for this pigeon in Experiment 1B (see Table 2).

\section{Results and Discussion}

Response and reinforcement rates in each schedule component in the last six sessions of the Training phase, and all sessions of the Response-Elimination and Resurgence phases are shown in Figure 18, for Pigeons 60 and 72. For both pigeons, reinforcement rates during the Training phase were similar in each component, and approximated the programmed rate of two reinforcers per minute. In this phase, response rates were stable (without downward trends) and similar in each component, for Pigeon 60, and were slightly higher in one component (later the rich component) for Pigeon 72. For this pigeon, response rates in both components had an increasing trend during the last six sessions of the Training phase. 
During the Response-Elimination phase, rate of responding was reduced in both components to near zero, after three sessions, for Pigeon 60. Consequently, reinforcement rates in the lean and rich components for this pigeon approximated the programmed rate of one and three reinforcers per minute. For Pigeon 72, responding in this phase also was reduced to near zero in each component, but after relatively more sessions than for Pigeon 60. Due to low rate responding of Pigeon 72 during the Response-Elimination phase, reinforcement rates were not as controlled as for Pigeon 60, although they were higher in the rich than in the lean component.

Resurgence occurred in both components only for Pigeon 60. Relative to the last sessions of the Response-Elimination phase, rate of responding increased in both rich and lean components for this pigeon, especially later in the Resurgence phase (e.g., in Session 4, and also in Sessions 7 and 10). As shown in Figure 18, and also in Figure 19 - which shows the frequency of key pecking in each component during the last six sessions of ResponseElimination phase, and all sessions of the Resurgence phase - no systematic relation between resurgence and reinforcement rate of alternative responding occurred. In addition, as occurred in Experiments 1-4, when differential resurgence occurred for this pigeon (in Sessions 4, 7 and 10 of the Resurgence phase), although more responding occurred in the rich than in the lean component, these differences were generally small (e.g., Sessions 4 and 10; see also, in Figures 5 and 6, similar results for this pigeon and for Pigeon 1, in Experiment 1B). For Pigeon 72, key pecking occurred at low frequencies across Resurgence-phase sessions, and more responding occurred in the rich than in the lean component. As with Experiments 1-4, the Resurgence-phase results had no direct relation to the component that was presented first in a session (see Table 3).

The results of this experiment were similar to those of Experiments $1 \mathrm{~B}$ through 4, in which variable-DRO schedules were in effect. Little to no resurgence occurred in each schedule 
component in these experiments (e.g., Mulick et al., 1976), and no relation between resurgence and reinforcement rates during the Response-Elimination phase was observed. Thus, the effects on resurgence of DRO-reinforcement rates in the present study were not a function the periodicity of reinforcement (i.e., fixed vs. variable IRIs). Doughty et al. (2007; Experiment 3) compared the effects on resurgence of variable and fixed-DROs and also found no differential effects of schedule type (although in their study, reinforcement rates in each component were equated, and resurgence was consistently observed within and across subjects).

In addition, the results were similar across experiments in the present study even though, in this experiment, the pigeons were exposed to fewer sessions during the Response-Elimination phase (they were not exposed to a multiple DRO $30 \mathrm{~s}$ DRO $30 \mathrm{~s}$ for 10-15 sessions). Although limited by exposing Pigeons 60 and 72 to similar stimulus conditions in this and in Experiments 1B and 4, respectively (see Table 2), the results were similar for these pigeons in each of these experiments and suggest that the periodicity of reinforcement in DRO schedules and the number of sessions in the Response-Elimination phase are not sufficient to account for the results obtained in Experiments 1B through 4.

In summary, little to no resurgence was obtained across Experiments 1 through 5. The use of DRO schedules (fixed or variable) might have been responsible for these results, even though it has been suggested that using these schedules increases the probability of resurgence (cf., Doughty et al., 2007; but see Mulick et al., 1976; see also Pacitti \& Smith, 1977 for differences in resurgence when DROs and other schedules of reinforcement were in effect in the Response-Elimination phase). This last aspect was studied in Experiment 6 by reinforcing, in each multiple-schedule component, an alternative key pecking response according to a VI schedule in the Response-Elimination phase. Similar to Experiment 5, fewer sessions were 
conducted in each phase and, similar to each of the previous experiments in the present study, the same schedule parameters were in effect to arrange differential reinforcement rates of alternative responding (i.e., $20 \mathrm{~s}$ and $60 \mathrm{~s}$ ).

\section{Experiment 6}

The use of DRO schedules (Doughty et al., 2007; see also da Silva et al., 2008) in the Response-Elimination phase does not allow the direct control of topography of alternative responding (which, in the present experiments, was defined as pausing; cf. Lattal \& Boyer, 1980; and has been described as a variable that affects resurgence, e.g., Doughty et al.; Pacitti \& Smith, 1977; but see Leitenberg et al., 1975, Experiment 2; and Winterbauer \& Bouton, 2010, Experiment 4). The present experiment was conducted to assess the effects of reinforcement rates of alternative responding on resurgence when alternative responding consisted of pecking a different key in each component of the multiple schedule. Procedurally, it approximates the analyses of Experiments 1 through 5 and previous resurgence studies (e.g., Leitenberg et al., 1975; Lieving \& Lattal, 2003; Podlesnik et al., 2006; Podlesnik \& Shahan, 2009, 2010;

Winterbauer \& Bouton, 2010) in which direct control of the topography of alternative responding was obtained by differentially reinforcing a topographically defined response during the Response-Elimination phase. In addition, the effects of exposing each pigeon to 25 sessions in the Response-Elimination phase in Experiments 1 through 4 (but see the procedure for Pigeons 806, in Experiment 2, and 828, in Experiment 4, for exceptions) was further assessed in this experiment by reducing the number of sessions in this phase. 


\section{Method}

\section{Subjects and Apparatus}

Six male White Carneau pigeons (Pigeons 56, 1 and 873, which served in Experiment 1B, and Pigeons 2, 12 and 828, which served in Experiment 4) were maintained as described in Experiment 1. The apparatus and stimulus conditions were as described in Experiment 1, with the following exceptions. Three operant chambers containing three keys were used and, for each pigeon, sessions were conducted in the chambers to which they were exposed in Experiments 1B and 4. In the Training phase, the center key was used (see Table 2 for the keylight colors used in the center key, for each pigeon) and during the Response-Elimination phase the right key in each chamber also was used and was transilluminated amber (for Pigeons 12, 56, 873 and 828) and green (for Pigeons 1 and 2).

\section{Procedure}

As in Experiment 5, the Training, Response-Elimination and Resurgence phases were in effect, respectively, for 10,15 and 10 sessions. In the Training phase, which was conducted as described in Experiments 1 through 4, center-key pecking was reinforced. During the ResponseElimination phase, reinforcers for center-key pecking were discontinued and VI schedules were in effect in the right key in each multiple-schedule component. During each component presentation in this phase, both the center and right keys were on, and each component of the multiple schedule was a concurrent extinction VI schedule (see Table 1). The right key was illuminated with the same keylight color in both components, which was different from the center keylights used for each pigeon (see Table 2).

As in Experiments 1 through 5, the VI parameters were $20 \mathrm{~s}$ in one (rich) component and $60 \mathrm{~s}$ in the other (lean) component, yielding a programmed reinforcement rate of three and one 
reinforcers per minute, respectively. The rich and lean components during the ResponseElimination phase were those with higher and lower response rates during the Training phase, respectively. In addition, a 3-s pause-response changeover delay (COD; Shahan \& Lattal, 1998) was in effect during this phase such that reinforcers were produced in each component only when right-key pecking occurred at least 3-s from the last key peck on the center key. The Resurgence phase was as described in Experiments 1 through 5, except that the center and right keys were on during each component presentation.

\section{Results and Discussion}

Figure 20 shows, for each pigeon, response and reinforcement rates in each component during the last six sessions of the Training phase and all sessions of the Response-Elimination and Resurgence phases. Closed and open symbols represent the lean and the rich components, respectively. In the response-rate graphs, circles and triangles show response rates on the center and right keys, respectively. For each pigeon, during the last sessions of the Training phase, similar rates of responding and reinforcement were obtained between components. In these sessions, responding was relatively stable in each component (without downward trends) and reinforcement rates approximated the programmed rate of two reinforcers per minute, for each pigeon.

During the Response-Elimination phase, the rate of center-key pecking was reduced and, for each pigeon, rates of right-key pecking increased in both components. With the exception of Pigeons 56 and 1, rates of right-key pecking were generally higher in the rich than in the lean component. In addition, reinforcement rates in this phase approximated the programmed rate of one and three reinforcers per minute in each component, for each pigeon. After 15 sessions, 
rates of responding on the center key were zero or near zero, for each pigeon, in both components.

Thus, the schedules of reinforcement in effect during the Response-Elimination phase of the present experiment were successful in reducing the frequency of center-key pecking and in establishing right-key pecking in each component. Compared to the DRO schedules in the previous experiments in the present study, the VI schedules were effective in establishing control of reinforcement rate of alternative responding. With the exception of Pigeons 1, 873 and 2 in the first session of the Response- Elimination phase, reinforcement rates approximated the programmed rate in each component during all sessions of this phase, for each pigeon.

In the Resurgence phase, rates of right-key pecking decreased and, during the initial sessions of this phase, were higher in the rich than in the lean component for each pigeon. When resurgence occurred in the present experiment, similar to what was observed in Experiments 1 through 5, no systematic differences between rich and lean components were observed. In some sessions, more center-key pecking occurred in the rich component, in others, in the lean. Data compression due to the Y-axis scale in response-rate graphs masks this effect in this figure for all pigeons except Pigeon 1.

These results can be seen in Figure 21, which shows the frequency of center-key pecking in each component during the last six sessions of the Response-Elimination phase and all sessions of the Resurgence phase, for each pigeon. Resurgence occurred in both the rich and lean components for Pigeons 1 and 2 (see also Appendix B for an analysis of these results according to Shahan \& Sweeney's [2011] model of resurgence). Differences in key-pecking frequency between components were not related to differences in reinforcement rates of alternative responding, nor they were extreme (e.g., as reported by Winterbauer \& Bouton, 
2010). For Pigeons 828 and 12, resurgence occurred in the rich component in some sessions and center key pecking in the lean component occurred at frequencies similar to, or lower than, those observed for these pigeons in the Response-Elimination phase (and, thus, did not characterize resurgence). The opposite outcome was obtained with Pigeon 56, for which resurgence was observed in the lean component but frequencies of key pecking were similar to, or lower than, those in the Response-Elimination phase in the rich component. Almost no center-key responding occurred in either component for Pigeon 873, although responding in the lean component during the last session of this phase could be described as resurgence.

The results of this experiment were similar to those of Experiments 1B through 5, independent of exposing the pigeons to (a) a reduced number of sessions during the ResponseElimination phase (see Lieving \& Lattal, 2003, Experiment 1; but see Cleland et al., 2000), (b) to a different schedule of reinforcement of alternative responding, relative to that arranged in the previous experiments in the present study (e.g., Leitenberg et al., 1975, Experiment 2; Mulick et al, 1976; see also Winterbauer \& Bouton, 2010, Experiment 4) and (c) of reinforcing a topographically-defined alternative response according to the same schedule parameters as in the previous experiments in the present study (but see Doughty et al., 2007; see also Pacitti \& Smith, 1977). In the present experiment, resurgence occurred in both components for only two of six pigeons and responding during the Resurgence phase was not systematically related to reinforcement rates of alternative responding (nor to which component was presented first in Resurgence-phase sessions; see Table 3).

The similar results between experiments in the present study suggest that the length of the Response-Elimination phase (e.g., Lieving \& Lattal, 2003, Experiment 1; but see Leitenberg et al., 1975, Experiment 4), and the schedules of reinforcement in effect across Response- 
Elimination phases in the present experiments (fixed- and variable DRO schedules, and a VI for pecking a different key) are not sufficient to account for the inconsistencies between this and previous resurgence studies (e.g., Doughty et al., 2007; Leitenberg et al., 1975, Experiment 3 and 4). Also, as indicated previously, a common factor in each of the present experiments (and one variable that differs from this and previous resurgence studies [e.g., Leitenberg et al], except the study by Mulick et al. [1976], in which results similar to those described herein were reported) is the schedule parameters in the Response-Elimination phase relative to those in the Training phase (i.e., VI $30 \mathrm{~s}$ during the Training phase, and DRO or VI $20 \mathrm{~s}$ and $60 \mathrm{~s}$, during the Response-Elimination phase).

That no parametric manipulations were conducted in the present study renders attributing the results obtained in Experiments 1B through 6 to these schedule parameters speculative. As suggested by Shahan and Sweeney (2011; see Appendices A and B) this might be a relevant variable that deserves further study before statements that reinforcement rates of alternative responding have no effect on resurgence. Such parametric analyses have not been reported, and previous resurgence studies in which reinforcement rates of alternative responding have been manipulated used primarily between subject comparisons (e.g., Leitenberg et al., 1975). The results of the present experiment, and of Experiments 1B through 5, eliminated other variables (such as the order of exposure to conditions in the Response-Elimination phase, the schedules of reinforcement and the topography of alternative responding) as explanations for the present results, and also suggest that schedule parameters might have affected responding in each schedule component during Resurgence-phase sessions and that this variable deserves further study. In the present experiments, only a few schedule parameters were studied (mainly, a DRO or VI $20 \mathrm{~s}$ and $60 \mathrm{~s}$ - or DRO $15 \mathrm{~s}$ and $90 \mathrm{~s}$, in Experiment 2 - relative to VI 30-s schedules in 
each schedule component during the Training phase). Replications of the present study (particularly of Experiment 6) in which reinforcement rates during the Training and ResponseElimination phases are parametrically manipulated would be interesting to further assess the effects of this variable on resurgence.

\section{General Discussion}

The present experiments were conducted to assess the effects of reinforcement rate of alternative responding on resurgence. Procedurally, they extend previous between-subject analyses (e.g., Leitenberg et al., 1975, Experiment 3; Winterbauer \& Bouton, 2010, Experiments 1 and 2) in that reinforcement rates of alternative responding were arranged in different components of a multiple schedule of reinforcement during the Response-Elimination phase of each experiment. In general, in the absence of systematic differences in response rates between schedule components in the Training phase (see Experiments 1A and 1B; cf. da Silva et al., 2008), little to no resurgence occurred in Experiments 1B through 6. In these experiments, resurgence was not related to the rates of reinforcement of alternative responding and, during some Resurgence-phase sessions, responding between components was not differential (e.g., Mulick et al., 1976; Winterbauer \& Bouton, 2010; Experiments 1 and 2). These results were not a function of which component was presented first during Resurgence-phase sessions (cf. da Silva et al.; see Table 3), nor of the order of component presentation within these sessions (see Figures 4 and 8 in Experiment 1).

In addition, the results were similar across Experiments 1 through 6 when (a) different schedules of reinforcement and topographies of alternative responding were in effect during the Response-Elimination phase (i.e., variable-DROs in Experiments 1 through 4; fixed-DROs, in Experiment 5, and a VI for pecking a different key, in Experiment 6); (b) the pigeons were 
exposed first to extinction and then to differential reinforcement rates of alternative responding (Experiment 3), or to differential and then, equal reinforcement rates of alternative responding during the Response-Elimination phase (Experiment 4); (c) a reduced number of sessions was conducted in each phase, particularly the Response-Elimination phase (Experiments 5 and 6). None of the current theoretical models of resurgence (e.g., Cleland et al., 2001; Leitenberg et al., 1975; Shahan \& Sweeney, 2011; see Appendix A; Winterbauer \& Bouton, 2010) predicted or accounted for these results. Each of these considerations is discussed below.

\section{Schedules of Reinforcement and Topography of Alternative Responding}

Neither the schedules of reinforcement nor the topography of alternative responding during the Response-Elimination phase affected resurgence differentially in the present study (Leitenberg et al., 1975, Experiment 2; Mulick et al., 1976; Winterbauer \& Bouton, 2010, Experiment 4; but see Doughty et al., 2007; and Pacitti \& Smith, 1977). Similar results were obtained when either variable- (Experiments 1 through 4) or fixed-DRO schedules (Experiment 5) were in effect during the Response-Elimination phase (and alternative responding was defined as pausing, cf. Lattal \& Boyer [1980], but its topography not directly controlled) and when the topography of alternative responding was a key peck maintained by a VI schedule (Experiment 6). These results are similar to those reported by Mulick et al. (1976), but are not consistent with those reported by Doughty et al. (2007), who, as noted above, found differential resurgence as a function of the topography of alternative responding, relative to the responses reinforced during the Training phase.

The similarity between the results of Experiments 1B through 6 suggest that differences in topography of responding between Training and Response-Elimination phases are not always a predictor of resurgence, and especially of differential resurgence (cf. Doughty et al., 2007). 
Although the reasons for the differences in results between the present experiments and those reported by Doughty et al. are not clear, one procedural difference that might have affected the results is that, in the present study, reinforcement rates of alternative responding were manipulated between schedule components, and in Doughty et al., they were equated across Training and Response-Elimination phases, and also between components within each phase. These inconsistencies in results across studies (see also Mulick et al., 1976; Pacitti \& Smith, 1977; Winterbauer \& Bouton, 2010, Experiment 4) render further analysis of the effects of schedules of reinforcement (and schedule parameters) and the differences in topography of Training- and Resurgence-phase responding a fruitful line of research.

Finally, the failure to replicate the findings of Experiment 1A when Training-phase response rates were similar in both multiple-schedule components in Experiments 1B through 6 suggests that the Training-phase response rate differences in Experiment 1A affected those results, and supports previous findings that characteristics of Training-phase responding (e.g., rate, da Silva et al., 2008; and the temporal distribution of responding, Cançado \& Lattal, 2011) affect, and are good predictors of, resurgence.

\section{Order of exposure to conditions during the Response-Elimination phase}

In the present Experiments 3 and, particularly, 4, the order of conditions during the Response-Elimination phase did not produce different results relative to those observed in Experiments 1B, 2, 5 and 6 . The findings of little to no resurgence, and of nondifferential resurgence in Experiments 1B and 2, thus, cannot be attributed solely to exposing the pigeons to a multiple DRO 30-s DRO 30-s schedule in the initial sessions of the Response-Elimination phase and then to the differential reinforcement rates of alternative responding. As noted, in Experiment 3 little to no resurgence occurred when responding was first extinguished before 
differential reinforcement rates of alternative responding were in effect in each component in the Response-Elimination phase (see Figures 13 and 14).

In addition, as described in Experiment 4, different from studies in which effects of sequential exposure to conditions during the Training phase affected resurgence (i.e., in which primacy and recency effects have been reported; e.g., Mechner \& Jones, 2011; Reed \& Morgan, 2006), responding in the Resurgence-phase of that experiment was not related to the order of exposure to reinforcement rates of alternative responding in the Response-Elimination phase. These results might suggest that when Training-phase variables are relatively similar between schedule components, as in the present study, the order in which Response-Elimination phase variables are programmed does not systematically affect resurgence.

\section{Length of the Training and Response-Elimination Phases and Resurgence}

The length of the Training phase was approximately 25 sessions for each pigeon in Experiments 1 through 4, and it was 10 sessions in Experiments 5 and 6 (see Table 2). Although in previous studies the length of the Training phase was shown to affect resurgence (e.g., Bruzek et al., 2009; Experiment 2; Doughty et al., 2010), the similar results obtained in Experiments 1 through 4, and in Experiments 5 and 6 indicate that it did not do so in the present study. In addition, this suggests that the length of the Response-Elimination phase (which was 25 sessions in Experiments 1 through 4, and 15 sessions in Experiments 5 and 6) did not systematically affect resurgence in the present study. These results are similar to those reported by Lieving and Lattal (2003, Experiment 1; but see Cleland et al., 2000; and Leitenberg et al., Experiment 4) who also reported no differences in resurgence as a function of length of the ResponseElimination phase. 
The length of the Response-Elimination phase was fixed in each of the present experiments to equate the exposure of each pigeon to the conditions in effect in that phase. A limitation of using such a fixed-time interval criterion is that responding might not have stabilized at the low levels that were desirable before phases would have to be changed (cf. Perone, 1991). Although, in general, rate of responding in each schedule component was zero or near zero in the last sessions of the Response-Elimination phases, in some cases responding was not consistently reduced when conditions were changed (see, for example, data for Pigeon 775 in the first exposure and replication of Experiment 2, in Figures 9-12). For this reason, in future studies it might be preferable to continue the Response-Elimination phase until responding is reduced according to some preestablished criterion (e.g., Cançado \& Lattal, 2011; da Silva et al., 2008).

\section{Resurgence and the Schedule Parameters during the Response-Elimination Phase}

That little to no resurgence occurred across the present Experiments 1B through 6 does not allow statements that reinforcement rates of alternative responding do not affect resurgence. The schedule values during the Training phase (30 s variable-IRIs) and during the ResponseElimination phase (in general, $20 \mathrm{~s}$ and $60 \mathrm{~s}$ variable- or fixed- IRIs; but see replication of Experiment 2), and consequently, the programmed reinforcement rates during these phases, might have been responsible for the lack of resurgence in general, and also for the lack of differential resurgence in the present experiments.

In the present experiments, the schedules in the rich and lean components during the Response-Elimination phase might have arranged reinforcement rates that were functionally identical. It is possible that exposing the pigeons to different schedule values (arranging a larger difference in reinforcement rates between components) during the Response-Elimination phase 
would produce more, or even, differential resurgence (cf. Shahan \& Sweeney, 2011; see Appendix A and B). For example, in Leitenberg et al.'s (1975; Experiment 3) study, in which resurgence was systematically affected by the rates of reinforcement of alternative responding, VI $30 \mathrm{~s}$ and VI $240 \mathrm{~s}$ arranged reinforcement rates during the Response-Elimination phase (a difference in reinforcement rates considerably larger than arranged between schedule components in the present study).

Also as previously noted, no parametric analyses of the effects of reinforcement rates of alternative responding on resurgence have been published. Replications of the present experiments in which such analyses are conducted would be a useful line of research. In addition to being useful to understanding the effects of this variable on resurgence (which are inconsistent across studies, including the present data; e.g., Leitenberg et al., 1975; Mulick et al., 1976; Winterbauer \& Bouton, 2010), such parametric analyses could provide data that would help efforts to systematize the results of previous resurgence studies theoretically (e.g., Cleland et al., 2001; Shahan \& Sweeney, 2011).

\section{The Present Results in Theoretical Context}

Three theoretical models have been proposed to account for resurgence: (a) the prevention-of-extinction hypothesis of resurgence (Leitenberg et al., 1975; Experiment 4; see also Cleland et al., 2001 and Appendix A); (b) a model of resurgence based on behavioral momentum theory (Shahan \& Sweeney, 2011) and an account of resurgence based on renewal (Bouton, 2002, 2004; see Winterbauer \& Bouton, 2010, Experiment 1). In addition, in Experiment 1A, an interpretation of resurgence based on resistance to change of alternative responding (Nevin \& Grace, 2000), also based on behavioral momentum theory, was suggested. Each of these models is discussed in what follows, as they relate to the present results. 
Resistance to change of alternative responding. The data in Experiment 1A (see Figures 1 and 2) were interpreted to suggest an inverse relation between reinforcement rates of alternative responding and resurgence. That is, higher reinforcement rates in the ResponseElimination phase would establish alternative responding that was relatively more resistant to change (cf. Nevin \& Grace, 2000), thus, leading to lower frequencies of Training-phase responding in the rich than in the lean component when reinforcers were discontinued in the Resurgence phase.

This interpretation is likely false, because the results of Experiment 1A were not replicated in the subsequent experiments. Notably, when differences in response rates between components during the Training phase were reduced (see particularly Experiment 1B, but also Experiments 2 through 5), the pattern of differential resurgence of that experiment was not obtained. This also was observed in Experiment 6, when the topography of alternative responding was specified, and the rate of such responding was measured directly (instead of indirectly estimated, as done when DRO schedules are in effect).

The prevention-of-extinction hypothesis. The results of the present study do not support the prevention-of-extinction hypothesis of resurgence (Cleland et al., 2000, Cleland et al., 2001; Leitenberg et al., 1975; Rawson et al., 1977) described in the literature review (see also Appendix A). This account suggests that higher rates of reinforcement of alternative responding should prevent Training-phase responding of being extinguished relatively more than lower reinforcement rates. Thus, an inverse relation between resurgence and (a) reinforcement rates of alternative responding and (b) the frequency of Training-phase responding during the ResponseElimination phase might be expected based on this account. As has been noted, differential resurgence was not consistently observed in the present study. In addition, as shown in the 
analyses of total number of responses during Response-Elimination and Resurgence phases in Experiments 1 and 3 (see Figures 3, 7 and 15, for these results, respectively, in Experiments 1A, 1B and 3), no inverse relation between the number of responses during the Resurgence phase and in each component during the Response-Elimination phase was observed.

Another prediction based on of the prevention-of-extinction hypothesis (see Cleland et al., 2000 and Leitenberg et al., 1975, Experiment 4) is that the longer subjects are exposed to the Response-Elimination phase (and, thus, to sessions in which reinforcers for Training-phase responding are discontinued), the lower resurgence should be. As discussed previously, the length of the Response-Elimination phase did not affect resurgence in the present experiments, a finding also inconsistent with this account of resurgence. In other experiments as well, resurgence has been reported consistently after Response-Elimination phases considerably lengthier than those in the present study (e.g., Lieving \& Lattal, 2003, Experiment 1; see also Doughty et al., 2007) and after Training-phase responding has been extinguished before reinforcement of alternative responding was in effect (e.g., Bruzek et al., 2009; Epstein, 1983; Winterbauer \& Bouton, 2011).

Resurgence and behavioral momentum theory. The quantitative model of resurgence proposed by Shahan and Sweeney (2011; see Appendix A), based on behavioral momentum theory (Nevin \& Grace, 2000), predicts more resurgence when higher, rather than lower, reinforcement rates are in effect during the Response-Elimination phase. This prediction would be made also for the present Experiments 1 through 6, considering the schedules and schedule parameters used in the present study. Based on this model, a simulation of responding in Experiments 1 and 6, are shown in Figures A1 and A2, respectively, in Appendix A. As can be seen in both figures, more resurgence is predicted in the rich than in the lean components with 
the schedule parameters in effect in the present study (including those used in Experiments 2 through 5). Thus, qualitatively, the results of the present experiments are inconsistent with this model of resurgence.

Although the present experiments were not designed as a test of this model, the inconsistencies between the present results and the predictions of resurgence based on the model were assessed quantitatively, and the results are shown in Appendix B. The model was fitted to data from Experiments 1A (in which results that differed from the predictions of the model were obtained) and 6 (which, for procedural reasons, can be a more accurate and fair assessment of the quantitative predictions of the model; i.e., in which the schedules in effect during Training and Response-Elimination phases were as described by Shahan \& Sweeney, 2011; see Appendix A). As shown in Figures B1 through B4, the model did not provide a good description of the results of these experiments, although it did account for relatively high proportions of the variance in the data (between $55 \%$ and $94 \%$ of the variance, across subjects; see Appendix B for a detailed description).

Shahan and Sweeney (2011) argued that relative response rates (i.e., responding as a proportion of baseline [here, the Training phase]) should be the primary dependent variable in the analysis of resurgence. Even though this position is consistent with behavioral momentum theory (Nevin \& Grace, 2000), it might not be always useful when resurgence is studied and, especially, when resurgence is not differential between components of a multiple schedule (as in the present experiments). That is, even if differences in absolute response rates do not obtain between schedule components during the Resurgence phase, differential resurgence might be obtained when relative response rates are calculated if systematic differences in absolute response rates between schedule components are observed during the Training phase 
(constituting different denominators for calculations of relative response rates and, thus, producing differential resurgence in relative terms when, in absolute terms, none was observed). This is not to say that Shahan and Sweeney, or other authors working from the perspective of behavioral-momentum theory would do this (or would not provide absolute measures of behavior when relative measures are presented), but to note that care should be taken in interpreting resurgence under these conditions. In addition to analyzing resurgence measured in relative terms, it is also meaningful to study resurgence as a change from zero or near-zero rates of responding during the Response-Elimination phase. Doughty, Reed and Lattal (2004) made this point in a reinstatement study, and suggested that, especially from a clinical perspective, "it seems more meaningful to consider the current rate of response recovery, as opposed to its change from pretreatment level"' (p. 1122).

Renewal. Winterbauer and Bouton (2010) suggested that the controlling variables of resurgence would be similar to those observed when another form of response recovery, renewal, is studied. Renewal is the recurrence of previously reinforced responding occasioned by a change from the context in which responding is extinguished (Bouton, 2002, 2004; Winterbauer \& Bouton). For example, in ABA renewal (e.g., Podlesnik \& Shahan, 2009; Nakajima, Tanaka, Urushiara, \& Imada, 2000), responding is reinforced in Context A. Reinforcers subsequently are discontinued in Context B and, still in extinction, subjects are reexposed to Context A. Renewal is operationalized as an increase in responding in the second exposure to Context $\mathrm{A}$, relative to responding in Context B, given this change in context (i.e., from B to A) when extinction is in effect. Other types of renewal have been reported previously, specifically (considering the phases described in the above example, in which each letter represents a different stimulus context) $\mathrm{AAB}$ and $\mathrm{ABC}$ renewal (see Winterbauer \& Bouton). Thus, according to this account, 
resurgence would be better described as an $\mathrm{ABC}$ renewal effect, in which contexts would be defined by the stimuli, the schedules, the subject's responding and also the consequences (e.g., food) produced initially but discontinued in the Resurgence phase (Winterbauer \& Bouton).

At least as proposed by Winterbauer and Bouton (2010; see also Winterbauer \& Bouton, 2011) a renewal interpretation could be invoked to account for any instance of resurgence (including in the present study), because a change in context always occurs in the transition from the Response-Elimination to the Resurgence phase. One difficulty for a renewal-based account of resurgence as currently formulated would be to explain cases in which resurgence is not observed, even though the change in context always occurs - arguing that in such cases there has been no actual change in context, in spite of the change in conditions, would make the definition of context not only broad (as it is) but circular and not useful to an experimental analysis of resurgence. Thus, although renewal effects might be demonstrated to be involved in resurgence in future studies, the current definition of context (see especially Bouton, 2002, 2004) seems too general, making any renewal-based interpretation of resurgence difficult to test (cf. Shahan \& Sweeney, 2011, p. 105).

\section{Conclusions}

The experimental analysis of resurgence (Epstein, 1983; Lieving \& Lattal, 2003) is an important area of research in part because it is a mirror of the organism's behavioral history (specifically, of the conditions in effect during the Training and Response-Elimination phases in a three-phase procedure). Resurgence is a robust and replicable phenomenon (see Doughty \& Oken, 2008; and Lattal \& St Peter Pipkin, 2009, for reviews). Recent demonstrations of resurgence of different responses and characteristics of responses (e.g., response sequences, Reed \& Morgan, 2006; and temporal patterns of responding, Cançado \& Lattal, 2011) and of 
responding maintained by different consequences (e.g., responding maintained by negative reinforcement; Bruzek et al., 2009) enhance our understanding of this phenomenon. Additional systematic analyses of the controlling variables of resurgence could also serve as a basis for more effective applications (e.g., Lieving et al., 2004; Volkert et al., 2009).

The present experiments were conducted to systematically analyze the effects on resurgence of reinforcement rates of alternative responding programmed during the ResponseElimination phase. Little to no resurgence, and no systematic differential resurgence, was obtained as a function of manipulations of this variable. As discussed previously, across experiments these results could not be accounted for on the basis of differences in schedules of reinforcement, topography of alternative responding, order of exposure to conditions or the length of Training and Response-Elimination phases. The schedule values (Leitenberg et.1975; see especially Shahan \& Sweeney, 2011) used during the Response-Elimination phase in each of the present experiments were likely responsible for the present results in that differences in reinforcement rates between rich and lean components might not have functioned as such, thus, leading to no differential resurgence.

The present study offers a procedure by which the effects of reinforcement rates of alternative responding on resurgence could be parametrically assessed in future experiments, thus clarifying the present results, the inconsistencies in previous research findings (e.g., Leitenberg et al., 1975; Mulick et al., 1976; Winterbauer \& Bouton, 2010) and extending the implications of an experimental analysis of this variable to a better understanding of resurgence. 


\section{References}

Bachá-Mendez, G., Reid, A. K., \& Mendoza-Soylovna, A. (2007). Resurgence of integrated behavioral units. Journal of the Experimental Analysis of Behavior, 87, 5-24. doi:10.1901/jeab.2007.55-05

Baum, W. M. (1973). The correlation-based law of effect. Journal of the Experimental Analysis of Behavior, 20, 137-153. doi:10.1901/jeab.1973.20-137

Baum, W. M. (1974). On two types of deviation from the matching law: bias and undermatching. Journal of the Experimental Analysis of Behavior, 22, 231-242. doi:10.1901/jeab.1974.22-231

Bouton, M. E. (2002). Context, ambiguity and unlearning: Sources of relapse after behavioral extinction. Biological Psychiatry, 52, 976-986. doi:10.1016/S0006-3223(02)01546-9

Bouton, M. E. (2004). Context and behavioral processes in extinction. Learning \& Memory, 11, 485-494. doi:10.1101/1m.78804

Bouton, M. E. (2011). Learning and the persistence of appetite: Extinction and the motivation to eat and overeat. Physiology \& Behavior, 103, 51-58. doi: 10.1016/j.physbeh.2010.11.025

Bruzek, J. L., Thompson, R. H., \& Peters, L. C. (2009). Resurgence of infant caregiving responses. Journal of the Experimental Analysis of Behavior, 92, 327-343. doi:10/1901.jeab.2009-92-327

Carey, J. P. (1951) Reinstatement of previously learned responses under conditions of extinction: A study of "regression" [Abstract]. American Psychologist, 6, 284.

Cleland, B. S., Foster, T. M., \& Temple, W. (2000). Resurgence: The role of extinction. Behavioural Processes, 52, 117-129. doi:10.1016/S0376-6357(00)00131-5 
Cleland, B. S., Guerin, B., Foster, T. M., \& Temple, W. (2001). Resurgence. The Behavior Analyst, 24, 255-260.

da Silva, S. P., Maxwell, M. E., \& Lattal, K. A. (2008). Concurrent resurgence and behavioral history. Journal of the Experimental Analysis of Behavior, 90, 313-331. doi:10.1901/jeab.2008.90-313

Doughty, A. H., Cash, J. D., Finch, E. A., Holloway, C., \& Wallington, L. K. (2010). Effects of training history on resurgence in humans. Behavioural Processes, 83, 340-343. doi: 10.1016/j.beproc.2009.12.001

Doughty, A. H., da Silva, S. P., \& Lattal, K. A. (2007). Differential resurgence and response elimination. Behavioural Processes, 75, 115-128. doi:10.1016/j.beproc.2007.02.025

Doughty, A. H., Reed, P., \& Lattal, K. A. (2004). Differential reinstatement predicted by preextinction response rate. Psychonomic Bulletin \& Review, 11, 1118-1123.

Doughty, A. H., \& Oken, G. (2008). Extinction-induced response resurgence: A selective review. The Behavior Analyst Today, 9, 27-33. Retrieved from http://www.baojournal.com/VOL9/BAT\%209-1.pdf

Epstein, R. (1983). Resurgence of previously reinforced behavior during extinction. Behaviour Analysis Letters, 3, 391-397.

Epstein, R. (1985). Extinction-induced resurgence: Preliminary investigations and possible applications. The Psychological Record, 35, 143-153.

Epstein, R. (1996). Cognition, creativity \& behavior: Selected essays. Westport, CT: Praeger Fleshler, M., \& Hoffman, H. S. (1962). A progression for generating variable-interval schedules. Journal of the Experimental Analysis of Behavior, 5, 529-530. doi:10.1901/jeab.1962.5529 
Franks, G. J, \& Lattal, K. A. (1976). Antecedent reinforcement schedule training and operant response reinstatement in rats. Animal Learning \& Behavior, 4, 374-378.

Lattal, K. A., \& Boyer, S. S. (1980). Alternative reinforcement effects on fixed-interval performance. Journal of the Experimental Analysis of Behavior, 34, 285-296. doi: 10.1901/jeab.1980.34-285

Lattal, K. A., \& St. Peter Pipkin, C. (2009). Resurgence of previously reinforced responding: Research and application. The Behavior Analyst Today, 10, 254-266. Retrieved from http://www.baojournal.com/VOL-10/BAT\%2010-2.pdf

Leitenberg, H., Rawson, R. A., \& Bath, K. B. (1970). Reinforcement of competing behavior during extinction. Science, 169, 301-303.

Leitenberg, H., Rawson, R. A., \& Mulick, J. A. (1975). Extinction and reinforcement of alternative behavior. Journal of Comparative and Physiological Psychology, 88, 640652. doi: $10.1037 / \mathrm{h} 0076418$

Lieving, G. A., \& Lattal, K. A. (2003). Recency, Repeatability, and Reinforcer Retrenchment: An Experimental Analysis of Resurgence. Journal of the Experimental Analysis of Behavior, 80, 217-233. doi:10.1901/jeab.2003.80-217

Lieving, G. A., Hagopian, L. P., Long, E. S., \& O'Connor, J. (2004). Response-class hierarchies and resurgence of severe problem behavior. The Psychological Record, 54, 621-634.

Mechner, F., \& Jones, L. (2011). Effects of sequential aspects of learning history. Mexican Journal of Behavior Analysis, 37,109-138. doi:10.5514/rmac.v37.i1.24688

Mulick, J. A., Leitenberg, H., \& Rawson, R. A. (1976). Alternative response training, differential renforcement of other behavior, and extinction in squirrel monkeys (Saimiri sciureus). Journal of the Experimental Analysis of Behavior, 25, 311-320. 
doi:10.1901/jeab.1976.25-311

Nakajima, S., Tanaka, S., Urushiara, K., \& Imada, H. (2000). Renewal of extinguished leverpress responses upon return to the training context. Learning \& Motivation, 31, 416-431. doi: $10.1006 /$ lmot.2000.1064

Nevin, J. A., \& Grace, R. C. (2000). Behavioral momentum and the Law of Effect. Behavioral and Brain Sciences, 23, 73-130. doi:10.1017/S0140525X00002405

Pacitti, W. A., \& Smith, N. F. (1977). A direct comparison of four methods for eliminating a response. Learning and Motivation, 8, 229-237. doi:10.1016/0023-9690(77)90007-8

Perone, M. (1991). Experimental design in the analysis of free-operant behavior. In I. H. Iversen \& K. A. Lattal (Eds.), Experimental Analysis of Behavior, Part 1, (pp. 135-171). New York, NY: Elsevier.

Podlesnik, C. A., Jimenez-Gomez, C., \& Shahan, T. A. (2006). Resurgence of alcohol seeking produced by discontinuing non-drug reinforcement as an animal model of drug relapse. Behavioural Pharmacology, 17, 369-374. doi:10.1097/01.fbp.0000224385.09486.ba

Podlesnik, C. A., \& Shahan, T. A. (2009). Behavioral momentum and relapse of extinguished operant responding. Learning \& Behavior, 37, 357-364. doi:10.3758/LB.37.4.357

Podlesnik, C. A., \& Shahan, T. A. (2010). Extinction, relapse, and behavioral momentum. Behavioural Processes, 84, 400-411. doi:10.1016/j.beproc.2010.02.001

Rawson, R. A., \& Leitenberg, H. (1973). Reinforced alternative behavior during punishment and extinction with rats. Journal of Comparative and Physiological Psychology, 85, 593-600. doi:10.1037/h0035312

Rawson, R. A., Leitenberg, H., Mulick, J. A., \& Lefebvre, M. F. (1977). Recovery of extinction responding in rats following discontinuation of reinforcement of alternative behavior: A 
test of two explanations. Animal Learning \& Behavior, 5, 415-420.

Reed, P., \& Clark, C. (2011). Impact of intervening learning on resurgence in humans with autism spectrum disorder. Learning \& Behavior, 39, 163-170. doi:10.3758/s13420-0100014-2

Reed, P., \& Morgan, T. A. (2006). Resurgence of response sequences during extinction in rats shows a primacy effect. Journal of the Experimental Analysis of Behavior, 86, 307-315. doi:10.1901/jeab.2006.20-05

Reed, P., \& Morgan, T. A. (2007). Resurgence of behavior during extinction depends on previous rate of response. Learning \& Behavior, 35, 106-114.

Reynolds, G. S. (1964). Operant extinction near zero. Journal of the Experimental Analysis of Behavior, 7, 173-176. doi: 10.1901/jeab.1964.7-173

Schoenfeld, W. N., Cumming, W. W., \& Hearst, E. (1956). On the classification of reinforcement schedules. Proceedings of the National Academy of Sciences, 42, 563-570. doi: $10.1073 /$ pnas. 42.8 .563

Shahan, T., \& Chase, P. N. (2002). Novelty, stimulus control and operant variability. The Behavior Analyst, 25, 179-190.

Shahan, T., \& Lattal, K. (1998). On the functions of the changeover delay. Journal of the Experimental Analysis of Behavior, 69, 141-160. doi:10.1901/jeab.1998.69-141

Shahan, T.A. \& Sweeney, M. M. (2011). A model of resurgence based on behavioral momentum theory. Journal of the Experimental Analysis of Behavior, 95, 91-108. doi: 10.1901/jeab.2011.95-91

Thompson, R. H., Bruzek, J. L., \& Cotnoir-Bichelman, N. M. (2011). The role of negative reinforcement in infant caregiving: An experimental simulation. Journal of Applied 
Behavior Analysis, 44, 295-304. doi: 10.1901/jaba.2011.44-295

Volkert, V. M., Lerman, D. C., Call, N. A., \& Trosclair-Lasserre, N. (2009). An evaluation of resurgence during treatment with functional communication training. Journal of Applied Behavior Analysis, 42, 145-160. doi: 10.1901/jaba.2009.42-145.

Winterbauer, N. E., \& Bouton, M. E. (2010). Mechanisms of resurgence of an extinguished instrumental behavior. Journal of Experimental Psychology: Animal Behavior Processes, 36, 343-353. doi:10.1037/a0017365

Winterbauer, N. E., \& Bouton, M. E. (2011). Mechanisms of resurgence II: Responsecontingent reinforcers can reinstate a second extinguished behavior. Learning and Motivation, 42, 154-164. doi:10.1016/j.lmot.2011.01.002 
Table 1. Schedules of Reinforcement and Schedule Parameters in Effect in the Response-

Elimination Phase in Experiments 1 through 6.

\begin{tabular}{|c|c|c|}
\hline & \multicolumn{2}{|c|}{$\begin{array}{l}\text { RESPONSE ELIMINATION } \\
\text { Schedule of Reinforcement }\end{array}$} \\
\hline & Initial sessions (10) & Terminal sessions (15) \\
\hline $\begin{array}{l}\text { Experiment } 1 \mathrm{~A} \\
\text { Experiment } 1 \mathrm{~B}\end{array}$ & Multiple DRO $30 \mathrm{~s}$ DRO $30 \mathrm{~s}$ & Multiple DRO $20 \mathrm{~s}$ DRO $60 \mathrm{~s}$ \\
\hline \multirow[t]{2}{*}{ Experiment $2^{\mathrm{a}}$} & Multiple DRO $30 \mathrm{~s}$ DRO $30 \mathrm{~s}$ & Multiple DRO $30 \mathrm{~s}$ DRO $20 \mathrm{~s}$ \\
\hline & & Multiple DRO $30 \mathrm{~s}$ DRO $60 \mathrm{~s}$ \\
\hline \multirow[t]{2}{*}{$\begin{array}{l}\text { Experiment 2- } \\
\text { Replication }\end{array}$} & Multiple DRO 30 s DRO 30 s & Multiple DRO $30 \mathrm{~s}$ DRO $90 \mathrm{~s}$ \\
\hline & & Multiple DRO $30 \mathrm{~s}$ DRO $15 \mathrm{~s}$ \\
\hline \multirow[t]{2}{*}{ Experiment 3} & Multiple EXT EXT & Multiple DRO $20 \mathrm{~s}$ DRO $60 \mathrm{~s}$ \\
\hline & Initial sessions (15) & Terminal sessions (10) \\
\hline \multirow[t]{2}{*}{ Experiment 4} & Multiple DRO 20 s DRO 60 s & Multiple DRO $30 \mathrm{~s}$ DRO $30 \mathrm{~s}$ \\
\hline & \multicolumn{2}{|c|}{15 sessions } \\
\hline Experiment $5^{b}$ & \multicolumn{2}{|c|}{ Multiple DRO 20 s DRO $60 \mathrm{~s}$} \\
\hline Experiment $6^{\mathrm{c}}$ & \multicolumn{2}{|c|}{ Multiple concurrent EXT VI $20 \mathrm{~s}$ concurrent EXT VI $60 \mathrm{~s}$} \\
\hline
\end{tabular}

Note. In Experiments 1 through 6 a multiple VI $30 \mathrm{~s}$ VI $30 \mathrm{~s}$ was in effect in the Training phase and variable-DRO schedules were in effect in the Response-Elimination phase in Experiments 1 through 4. In the Resurgence phase of each experiment, reinforcers were discontinued in each schedule component.

${ }^{\mathrm{a}}$ In the replication of Experiment 2, a DRO $15 \mathrm{~s}$ and a DRO $90 \mathrm{~s}$ were in effect in one schedule component for pigeons that were exposed, respectively, to DRO 60-s and DRO 20-s schedules in the first exposure to the Response-Elimination phase. The component correlated with the DRO $30 \mathrm{~s}$ was the same during the first exposure and the replication of this experiment.

${ }^{\mathrm{b}}$ The IRIs in each DRO schedule were fixed in Experiment 5.

${ }^{\mathrm{c}}$ In the Response-Elimination phase of Experiment 6, extinction was in effect on the center key, and reinforcement was arranged according to VI schedules for pecking the right key in each schedule component. 
Table 2. Keylight Colors Correlated with the Rich and Lean Schedule Components in the

Response-Elimination Phase, and the Number of Sessions Conducted in the Training phase, for

Each Pigeon, in Experiments 1 through 6.

\begin{tabular}{|c|c|c|c|c|}
\hline & \multirow[t]{2}{*}{ Pigeon } & \multicolumn{2}{|c|}{ Key Light Color } & \multirow{2}{*}{$\begin{array}{c}\text { Training Phase } \\
\text { Sessions } \\
\end{array}$} \\
\hline & & Rich & Lean & \\
\hline \multirow{4}{*}{ Experiment $1 \mathrm{~A}$} & 617 & Red & Green & 27 \\
\hline & 681 & Green & Red & 23 \\
\hline & 627 & Red & Amber & 23 \\
\hline & 580 & Amber & Red & 23 \\
\hline \multirow{4}{*}{ Experiment 1B } & 56 & Green & Red & 20 \\
\hline & 1 & Amber & Red & 20 \\
\hline & 60 & Red & Amber & 20 \\
\hline & 873 & Red & Green & 20 \\
\hline \multirow{4}{*}{ Experiment $2^{\mathrm{a}}$} & 691 & Green & Red & 20 \\
\hline & 806 & Green & Red & 25 \\
\hline & 847 & Amber & Red & 20 \\
\hline & 775 & Red & Amber & 20 \\
\hline \multirow{4}{*}{ Experiment 3} & 617 & Green & Red & 20 \\
\hline & 874 & Red & Amber & 25 \\
\hline & 627 & Green & Red & 20 \\
\hline & 681 & Red & Amber & 20 \\
\hline \multirow{4}{*}{ Experiment 4} & 12 & Red & Green & 20 \\
\hline & 2 & Red & Amber & 20 \\
\hline & 72 & Red & Amber & 20 \\
\hline & 828 & Red & Green & 25 \\
\hline \multirow[t]{2}{*}{ Experiment 5} & 72 & Red & Amber & 10 \\
\hline & 60 & Amber & Red & 10 \\
\hline \multirow{6}{*}{ Experiment $6^{\mathrm{b}}$} & 56 & Red & Green & 10 \\
\hline & 1 & Amber & Red & 10 \\
\hline & 873 & Green & Red & 10 \\
\hline & 2 & Red & Amber & 10 \\
\hline & 12 & Green & Red & 10 \\
\hline & 828 & Green & Red & 10 \\
\hline
\end{tabular}

${ }^{\text {a }}$ In the replication of Experiment 2, the keylights correlated with each component were reversed and, for each pigeon, 20 sessions were conducted during the Training phase.

${ }^{\mathrm{b}}$ In Experiment 6, the right key was transilluminated amber, for Pigeons 12, 56, 873 and 828, and green, for Pigeons 1 and 2. 
Table 3. First Component, and Frequency of Rich and Lean Components, in Each Resurgence-

Phase Session, for Each Pigeon in Experiments 1 through 6.

\begin{tabular}{|c|c|c|c|c|c|c|c|c|c|c|c|c|}
\hline \multicolumn{2}{|c|}{ Experiment/Pigeon } & \multicolumn{10}{|c|}{ First component in Resurgence-phase session } & \multirow{2}{*}{$\begin{array}{l}\text { Frequency } \\
\text { Rich/Lean }\end{array}$} \\
\hline \multirow{5}{*}{$1 \mathrm{~A}$} & & S1 & S2 & S3 & S4 & S5 & S6 & S7 & S8 & S9 & S10 & \\
\hline & 617 & Rich & Lean & Lean & Rich & Rich & Lean & Lean & Lean & Rich & Rich & $5 / 5$ \\
\hline & 681 & Rich & Lean & Rich & Rich & Lean & Rich & Rich & Lean & Lean & Rich & $6 / 4^{\mathrm{a}}$ \\
\hline & 627 & Lean & Lean & Rich & Rich & Lean & Lean & Rich & Rich & Rich & Lean & $5 / 5$ \\
\hline & 580 & Rich & Lean & Lean & Lean & Rich & Rich & Rich & Lean & Lean & Rich & $5 / 5$ \\
\hline \multirow{4}{*}{ 1B } & 56 & Lean & Lean & Rich & Lean & Rich & Rich & Rich & Lean & Lean & Rich & $5 / 5$ \\
\hline & 1 & Lean & Rich & Lean & Lean & Rich & Rich & Lean & Rich & Rich & Lean & $5 / 5$ \\
\hline & 60 & Rich & Lean & Rich & Rich & Lean & Lean & Rich & Lean & Lean & Rich & $5 / 5$ \\
\hline & 873 & Rich & Lean & Rich & Lean & Rich & Rich & Lean & Lean & Lean & Rich & $5 / 5$ \\
\hline \multirow{4}{*}{$\begin{array}{c}\text { 2; First } \\
\text { Exposure }\end{array}$} & 691 & Lean & Rich & Rich & Rich & Lean & Lean & Lean & Rich & Rich & Lean & $5 / 5$ \\
\hline & 806 & Lean & Lean & Lean & Rich & Rich & Lean & Rich & Rich & Lean & Rich & $5 / 5$ \\
\hline & 847 & Lean & Lean & Rich & Lean & Rich & Lean & Lean & Rich & Rich & Lean & $4 / 6^{\mathrm{a}}$ \\
\hline & 775 & Rich & Rich & Rich & Lean & Lean & Lean & Rich & Rich & Lean & Rich & $6 / 4^{\mathrm{a}}$ \\
\hline \multirow{4}{*}{$\begin{array}{c}2 ; \\
\text { Replication }\end{array}$} & 691 & Rich & Lean & Rich & Lean & Lean & Rich & Rich & Rich & Lean & Lean & $5 / 5$ \\
\hline & 806 & Lean & Rich & Lean & Lean & Rich & Rich & Lean & Rich & Lean & Rich & $5 / 5$ \\
\hline & 847 & Rich & Lean & Lean & Rich & Lean & Lean & Rich & Lean & Rich & Rich & $5 / 5$ \\
\hline & 775 & Rich & Rich & Lean & Lean & Lean & Rich & Lean & Lean & Rich & Rich & $5 / 5$ \\
\hline \multirow{4}{*}{3} & 617 & Lean & Rich & Rich & Lean & Lean & Rich & Rich & Lean & Rich & Lean & $5 / 5$ \\
\hline & 874 & Rich & Lean & Rich & Lean & Rich & Rich & Rich & Lean & Lean & Lean & $5 / 5$ \\
\hline & 627 & Rich & Lean & Lean & Rich & Rich & Lean & Rich & Lean & Rich & Lean & $5 / 5$ \\
\hline & 681 & Rich & Rich & Lean & Rich & Lean & Lean & Lean & Rich & Lean & Rich & $5 / 5$ \\
\hline \multirow{4}{*}{4} & 12 & Rich & Lean & Lean & Rich & Rich & Lean & Rich & Rich & Lean & Lean & $5 / 5$ \\
\hline & 2 & Rich & Lean & Rich & Rich & Lean & Lean & Rich & Lean & Rich & Lean & $5 / 5$ \\
\hline & 72 & Lean & Rich & Lean & Lean & Rich & Rich & Lean & Rich & Lean & Rich & $5 / 5$ \\
\hline & 828 & Lean & Rich & Rich & Lean & Lean & Lean & Rich & Rich & Lean & Rich & $5 / 5$ \\
\hline \multirow[t]{2}{*}{5} & 60 & Rich & Lean & Rich & Lean & Rich & Lean & Rich & Rich & Lean & Lean & $5 / 5$ \\
\hline & 72 & Lean & Lean & Rich & Lean & Lean & Rich & Rich & Rich & Lean & Rich & $5 / 5$ \\
\hline \multirow{6}{*}{6} & 56 & Rich & Lean & Rich & Rich & Lean & Rich & Lean & Lean & Lean & Rich & $5 / 5$ \\
\hline & 1 & Rich & Lean & Rich & Lean & Rich & Lean & Lean & Lean & Rich & Rich & $5 / 5$ \\
\hline & 873 & Lean & Rich & Lean & Rich & Rich & Rich & Lean & Rich & Lean & Lean & $5 / 5$ \\
\hline & 2 & Lean & Rich & Lean & Rich & Rich & Rich & Lean & Rich & Lean & Lean & $5 / 5$ \\
\hline & 12 & Rich & Lean & Lean & Rich & Lean & Rich & Lean & Rich & Lean & Rich & $5 / 5$ \\
\hline & 828 & Lean & Rich & Rich & Lean & Rich & Lean & Lean & Rich & Lean & Rich & $5 / 5$ \\
\hline
\end{tabular}

${ }^{a}$ The number of sessions starting with rich and lean components was not equal (i.e., 5/5). 


\section{Appendix A}

The quantitative model of resurgence proposed by Cleland et al. (2001) is based on the generalized matching law (Baum, 1973, 1974). The main assumptions made by Cleland et al. are that (a) the occurrence of Training-phase responding during the Response-Elimination phase is a function of reinforcement rates during both the Training and Response-Elimination phases; and that (b) resurgence is an inverse function of the occurrence of Training-phase responding during the Response-Elimination phase (i.e., a formal statement of the prevention-of-extinction hypothesis of resurgence, Leitenberg et al., 1975; Rawson et al., 1977). Formally (p. 259),

$$
\log \left(\frac{B_{1 t}}{B_{1 e}}\right)=a \log \left(\frac{r 1}{r 2}\right) k
$$

where, $B_{1 t}$ and $B_{l e}$ refer to, respectively, the occurrence of Training-phase responding during the Response-Elimination and the Resurgence phases, respectively. Reinforcement rates during these phases are represented by, respectively, $r 1$ and $r 2$ and the parameters $a$ and $k$ quantify, respectively, sensitivity of behavior allocation to reinforcement ratios and bias (cf., Baum 1974).

According to Equation A1, the occurrence of Training-phase responding during the Response-Elimination and Resurgence phases is a function of the proportion of reinforcement rates during Training and Response-Elimination phases $(r 1 / r 2)$. That is, more and less Trainingphase responding is predicted during the Response-Elimination phase if $r 1>r 2$ and if $r 2>r 1$, respectively. Additionally, more resurgence is predicted when $r 2>r 1$. It should be noted that, according to this model, resurgence is inversely related to the rate of reinforcement during the Training phase (i.e., r1; cf. Shahan \& Sweeney, 2011) the opposite of what has been previously reported (see especially Podlesnik \& Shahan, 2009, 2010).

Shahan and Sweeney (2011) proposed a quantitative model of resurgence based on behavioral momentum theory (more specifically, in the augmented model of extinction proposed 
by Nevin \& Grace, 2000). Procedurally, it assumes that the three-phase procedure used in the study of resurgence (i.e., Training, Response-Elimination and Resurgence phases) is conducted such that: a response is reinforced during the Training phase and, during the ResponseElimination phase, this first response is extinguished while an alternative response is concurrently reinforced. Based on previous attempts to model resurgence by Podlesnik and Shahan $(2009,2010)$, the model was formally stated as,

$$
\log \frac{B_{t}}{B_{o}}=\frac{-t\left(k R_{a}+c+d r\right)}{\left(r+R_{a}\right)^{b}}
$$

where $B_{t}$ is the rate of Training-phase responding at time $t$ (measured as sessions in which reinforcers for this response are discontinued) and $B_{o}$ is the rate of responding during the Training phase. The parameter $c$ quantifies the disruption of eliminating the response-reinforcer contingency when reinforcers are discontinued for Training-phase responding in the ResponseElimination phase and the parameter $d$ scales generalization decrement. In the model, reinforcement rates are calculated in reinforcers per hour; $r$ is the rate of reinforcement of responding during the Training phase and $R_{a}$ is the rate of reinforcement of alternative responding. The parameter $b$ is sensitivity of Training-phase responding to the total reinforcement rate and the parameter $k$ scales the disruption in Training-phase responding caused by $R_{a}$ in the Response-Elimination phase (it should be noted that, for all analyses of previously published data and simulations based on their model, Shahan and Sweeney, p.94, treated the parameters $c$ and $k$ as free-parameters, but $b$ and $d$ were constants - with values set as, respectively, 0.5 and 0.001$)$.

According to Equation A2, more resurgence is predicted when responding is maintained by higher reinforcement rates during the Training phase. There is also an inverse relation between the number of sessions during Response-Elimination and Resurgence phases and the 
magnitude of resurgence, i.e., other things being constant, the higher the value of $t$, the greater the disruption in responding caused by the numerator in Equation 2 and, thus, less resurgence is predicted.

The main assumption of Shahan and Sweeney's (2011) model is that $R_{a}$ contributes to the reduction in rate of the Training-phase responding (see the numerator, or disruptor term, of Equation A2) and also to increase its strength and subsequent resurgence when reinforcers for alternative responding are discontinued (the denominator of Equation A2). Different from Cleland et al.'s (2001) model, a direct relation between resurgence and Training-phase reinforcement rates is stated. Similar to Cleland et al.'s model (but based on the assumption of a strengthening effect of $R_{a}$ on Training-phase responding, cf. Nevin \& Grace; and not on the prevention-of-extinction of this responding by $R_{a}$, cf. Leitenberg et al., 1975) a direct relation between $R_{a}$ and resurgence is stated.

Shahan and Sweeney (2011) described an exponentiated version of Equation A2, which, different from that equation, allows for the inclusion of zero values in the analyses:

$$
\frac{B_{t}}{B_{o}}=10^{\frac{-t(k R a+c+d r)}{(r+R a)^{b}}}
$$

where all terms are as in Equation A2. The authors also described a version of the model that can be used to describe absolute response rates (see their Equation 5, p. 94), although they emphasized that, according to behavioral momentum theory, relative response rates are the appropriate measure of resistance to change and resurgence.

In simulations of the model in which the value of $R_{a}$ was systematically increased, the differences in resurgence between components of a multiple schedule decreased (see Shahan \& Sweeney's Figure 4, p. 100). According to their model, the relation between the schedule parameters during the Training and Response-Elimination phases (i.e., the relative values of $r$ 
and $R_{a}$ ) affects the magnitude of resurgence and also the degree of differential resurgence. In each of Shahan and Sweeney's simulations, however, each components of a two-component multiple schedule programmed different reinforcement rates during the Training phase (i.e., a multiple VI 30 s VI 120 s) and schedule parameters were equal in each component (e.g., VI 240 s, VI 120 s, VI 60 s, VI 30 s or VI 15 s) during the Response-Elimination phase. In the present study, the opposite was done (equal programmed reinforcement rates were in effect during the Training phase, and reinforcement rates of alternative responding differed between components during the Response-Elimination phase).

In Figure A1 a simulation of responding, based on Equation A3, is presented. Schedule parameters and procedure were as those arranged in Experiment 1 (a multiple VI 30s VI 30 s was in effect during Training and a multiple DRO $30 \mathrm{~s}$ DRO $30 \mathrm{~s}$, and a multiple DRO $20 \mathrm{~s} 60 \mathrm{~s}$, were in effect during the initial and terminal sessions of the Response-Elimination phase, respectively). Responding is shown in each component as a proportion of mean response rates during the last six sessions of the Training phase. Little to no responding is predicted during the Resurgence phase (which could be attributed also to the number of sessions conducted during the Response-Elimination phase), although the model predicts consistently more resurgence in the rich than in the lean component.

Similar results can be seen in Figure A2, which shows a simulation of responding in Experiment 6 based on Equation A3. Compared to the simulated data in Figure A1, more responding occurred in both components during the Resurgence phase, and more resurgence also was predicted in the rich than in the lean component (note that in Experiment 6, fewer sessions were conducted in the Response-Elimination phase). Thus, the model predicted a slight difference between components during the Resurgence phase, with more responding occurring 
consistently in the rich than in the lean component (see also Appendix B for fits of Equation A3 to data from Experiments $1 \mathrm{~A}$ and 6).

Finally, it should be noted that Shahan and Sweeney (2011) proposed a model in which alternative responding is topographically specified. Although they did not assume any differential effects of schedules of reinforcement during the Response-Elimination phase on resurgence, their model is not a model of DRO schedules (e.g., Doughty et al., 2007), as used in the present Experiments 1 through 5. 


\section{Appendix B}

In this section, fits of Shahan and Sweeney's (2011; see Appendix A for a detailed description) model to data from Experiments $1 \mathrm{~A}$ and 6 are presented. Although the results of the present study were qualitatively different from the model's predictions (see Figures A1 and A2), a more accurate assessment of how it described the results quantitatively required fitting it to some of the present results. Another reason for conducting this analysis is that, in the present experiments, Training-phase reinforcement rates were similar, and reinforcement rates of alternative responding were different, between multiple-schedule components - a manipulation not previously conducted, nor addressed by Shahan and Sweeney.

Relative response rates were calculated in each component, in Experiments $1 \mathrm{~A}$ and 6, for all sessions of the Response-Elimination and Resurgence phases (data points are proportions of mean response rates in each component during the last six sessions of the Training phase). Reinforcement rates in each component during the Training $(r)$ and Response-Elimination phases $\left(R_{a}\right)$ were calculated in reinforcers per hour (cf. Shahan \& Sweeney, 2011). The parameters $b$ and $d$ were set as equal to 0.5 and 0.001 , respectively (see Shahan $\&$ Sweeney, p. 94) and $k$ and $c$ were treated as free parameters. For each experiment, Equation A3 was fitted separately to responding in each component. Variance accounted for, and the $c$ and $k$ parameters, however, were estimated based on data from both rich and lean components. Fits of the model and parameter estimation were conducted by the method of least-squares regression, and by using Microsoft Excel 2007 ® and the data-analysis tool Solver in that software.

Figure B1 shows the fits of Equation A3 to data from Experiment 1A. Closed and open circles represent lean and rich components, respectively. In each graph, solid and dashed functions are best fits of Equation A3 to data from, respectively, the lean and rich components. 
In general, the model predicted more resurgence in the rich than in the lean component during all Resurgence-phase sessions, not the opposite pattern of differential resurgence obtained initially. Even though, as discussed previously (see the Results and Discussion sections of Experiments 1A and 1B), differences between components in Training-phase response rates affected these results, the model did not predict the non-differential responding during some sessions of the Resurgence phase that occurred for Pigeons 617, 627 and 580. Although the model accounted for a relatively high proportion of the variance in the data (particularly for Pigeons 681, 91\%, and $627,92 \%$ ), and the parameter values for these two pigeons approximated those reported by Shahan and Sweeney (i.e., values of $c$ and $k$ of approximately $0.89-1.00$ and 0.05 , respectively) it did not provide a good quantitative description of the results. Residual analyses (obtained predicted), shown in Figure B2, support this conclusion in that responding in the rich and lean components during the Resurgence phase was, respectively, over- and underestimated by the model (i.e., the negative and positive residuals were obtained in the rich and lean components during the Resurgence-phase sessions, respectively).

Similar results, in terms of variance accounted for, and parameter estimation, were obtained for fits of Equation A3 to data from Experiment 6 (only data for Pigeons 1 and 2 were included in this analysis, because resurgence occurred in both components during the Resurgence phase of that experiment only for these two pigeons). Fits of the model and residuals are shown in Figures B3 and B4, respectively. The model predicted more resurgence in the rich than in the lean component, and consistent differences in responding between components during the Resurgence phase, not the generally similar responding in both components during this phase for each pigeon. As shown in the residual analysis, the model also over- and underestimated the data in rich and lean components for these two pigeons. 
Although the model predicts that the degree of differential resurgence is affected by the schedule parameters during the Training and the Response-Elimination phases, it did not provide a good description of the results of the present experiments (see Figures A1 and A2). The results of the present study are limited in further assessing this model, however, because little to no resurgence occurred across experiments (see results of Experiments 1B through 6), and it was generally not differential between rich and lean schedule components. The adequacy of the model in accounting for the results of resurgence studies requires experiments specifically designed to test it, and a more extensive parametric analysis of the effects of reinforcement rates of alternative responding on resurgence (not conducted in the present experiment nor in any previously published study). 


\section{Figure Captions}

Figure 1. Response (left graphs) and reinforcement (right graphs) rates in each schedule component during the last six sessions of the Training phase and all sessions of the ResponseElimination and Resurgence phases of Experiment 1A. Dashed vertical lines in each graph indicate when reinforcement rate was manipulated in each component during the ResponseElimination phase. Closed and open circles show responses and reinforcers per minute in the lean and rich components, respectively. Note the different Y-axis scale in the response-rate graph for Pigeon 580.

Figure 2. Frequency of key pecking in each schedule component during the last six sessions of the Response-Elimination phase and all sessions of the Resurgence phase in Experiment 1A. Closed and open bars represent the lean and rich components, respectively. Note the different Yaxis scale for Pigeon 627.

Figure 3. Total number of key pecks in each schedule component during all sessions of the Response-Elimination and Resurgence phases, for each pigeon in Experiment 1A. The Y-axis in each graph is on logarithmic scale (base 10). Closed and open circles represent the lean and rich components, respectively.

Figure 4. Mean frequency of key pecking in each schedule component as a function of withinsession order of component presentation, for each pigeon in Experiment 1A. Mean frequencies are shown for Resurgence-phase sessions in which the first component was lean (left graphs) and rich (right graphs). In each graph, closed and open bars represent lean and rich components, respectively. Note the different Y-axis scale for Pigeon 681.

Figure 5. Response (left graphs) and reinforcement (right graphs) rates in each schedule component during the last six sessions of the Training phase and all sessions of the Response- 
Elimination and the Resurgence phases of Experiment 1B. Note the different Y-axis scale in the response-rate graph for Pigeon 873. Other details as in Figure 1.

Figure 6. Frequency of key pecking in each schedule component during the last six sessions of the Response-Elimination phase and all sessions of the Resurgence phase in Experiment 1B. Note the different Y-axis scales for Pigeons 60 and 1. Other details as in Figure 2.

Figure 7. Total number of key pecks in each schedule component during all sessions of the Response-Elimination and Resurgence phases, for Pigeons 1 and 60 in Experiment 1B. Other details as in Figure 3.

Figure 8. Mean frequency of key pecking in each schedule component as a function of withinsession order of component presentation, for Pigeons 1 and 60 in Experiment 1B. Note the different $\mathrm{Y}$-axis scale for each pigeon. Other details as in Figure 4.

Figure 9. Response (left graphs) and reinforcement (right graphs) rates in each schedule component during the last six sessions of the Training phase and all sessions of the ResponseElimination and Resurgence phases of Experiment 2 (first exposure). Pigeon 806 was exposed to 17 terminal sessions in the Response-Elimination phase. Other details as in Figure 1.

Figure 10. Frequency of key pecking in each schedule component during the last six sessions of the Response-Elimination phase and all sessions of the Resurgence phase in Experiment 2 (first exposure). Note the difference in Y-axis scale between upper (Pigeons 691 and 806) and bottom graphs (Pigeons 847 and 775). Other details as in Figure 2.

Figure 11. Response (left graphs) and reinforcement (right graphs) rates in each schedule component during the last six sessions of the Training phase and all sessions of the ResponseElimination and Resurgence phases of Experiment 2 (replication). Note the different Y-axis scale in the response rate-graph for Pigeon 847. Other details as in Figure 1. 
Figure 12. Frequency of key pecking in each schedule component during the last six sessions of the Response-Elimination phase and all sessions of the Resurgence phase in Experiment 2 (replication). Note the difference in Y-axis scale for Pigeon 806. Other details as in Figure 2. Figure 13. Response (left graphs) and reinforcement (right graphs) rates in each schedule component during the last six sessions of the Training phase and all sessions of the ResponseElimination and Resurgence phases of Experiment 3. Note the different Y-axis scale in the response-rate graph for Pigeon 627. Other details as in Figure 1.

Figure 14. Frequency of key pecking in each schedule component during the last six sessions of the Response-Elimination phase and all sessions of the Resurgence phase in Experiment 3. Note the different Y-axis scale for Pigeon 617. Other details as in Figure 2.

Figure 15. Total number of key pecks in each schedule component during all sessions of the Response-Elimination and Resurgence phases, for each pigeon in Experiment 3. Other details as in Figure 3.

Figure 16. Response (left graphs) and reinforcement (right graphs) rates in each schedule component during the last six sessions of the Training phase and all sessions of the ResponseElimination and Resurgence phases of Experiment 4. An asterisk in the response and reinforcement rate graphs for Pigeon 828 indicates the session in which a multiple VI 30 s VI 30 s was in effect during the Response-Elimination phase (see Method section of Experiment 4 for details). Note the different $\mathrm{Y}$-axis scale in the response-rates graphs for Pigeons 2 and 828 . Other details as in Figure 1.

Figure 17. Frequency of key pecking in each schedule component during the last six sessions of the Response-Elimination phase and all sessions of the Resurgence phase in Experiment 4. Note 
the difference in Y-axis scale between upper (Pigeons 12 and 2) and bottom graphs (Pigeons 72 and 828). Other details as in Figure 2.

Figure 18. Response (left graphs) and reinforcement (right graphs) rates in each schedule component during the last six sessions of the Training phase and all sessions of the ResponseElimination and Resurgence phases of Experiment 5. Note the difference in Y-axis scales between response-rate graphs for Pigeons 72 and 60. Other details as in Figure 1.

Figure 19. Frequency of key pecking in each schedule component during the last six sessions of the Response-Elimination phase and all sessions of the Resurgence phase in Experiment 5. Other details as in Figure 2.

Figure 20. Response (left graphs) and reinforcement (right graphs) rates in each schedule component during the last six sessions of the Training phase and all sessions of the ResponseElimination and Resurgence phases of Experiment 6. Closed and open triangles with dashed lines (left graphs) represent right-key pecking in the lean and rich components, respectively. Note the different Y-axis scales in the response-rate graphs for Pigeons 873, 2 and 12. Other details as in Figure 1.

Figure 21. Frequency of center-key pecking in each schedule component during the last six sessions of the Response-Elimination phase and all sessions of the Resurgence phase in Experiment 6. Note the different Y-axis scales for Pigeons 1, 873 and 828. Other details as in Figure 2 .

Figure A1. Simulation of responding in Experiment 1, based on Shahan and Sweeney's (2011) model (Equation A3). Relative response rates are shown in the lean (solid lines) and rich (dashed lines) components for all sessions of Response-Elimination and Resurgence phases. The simulation assumes a multiple VI 30 s VI 30 s during the Training phase and a multiple DRO 30 
s DRO 30 s, and a multiple DRO 20 s DRO 60 s during the initial and terminal sessions of the Response-Elimination phase, respectively.

Figure A2. Simulation of responding in Experiment 6 based on Shahan and Sweeney's (2011) model (Equation A3). The simulation assumes a multiple VI 30 s VI 30 s during the Training phase and a multiple concurrent EXT VI 20 s concurrent EXT VI 60 s during the ResponseElimination phase. Other details as in Figure A1.

Figure B1. Relative response rate in the lean (closed circles) and rich (open circles) schedule components during all sessions of Response-Elimination and Resurgence phases, for each pigeon in Experiment 1A. Vertical lines in each graph separate the first 10 and final 15 sessions of Response-Elimination and the Resurgence phase sessions. Data points are proportions of the mean response rate of the last six sessions of the Training phase. Solid and dashed lines are best fits of Equation A3 to data from the lean and rich schedule components, respectively. Note the different Y-axis scale for Pigeon 617.

Figure B2. Residuals (obtained - predicted) of fits of Equation A3 to data from all sessions of Response-Elimination and Resurgence phases, for each pigeon in Experiment 1A. In each graph, dashed vertical lines separate the first 10 and final 15 sessions of Response-Elimination and the Resurgence-phase sessions. Closed and open circles represent the lean and rich schedule components, respectively. Note the difference in Y-axis scales between upper (Pigeons 617 and 681) and bottom graphs (Pigeons 627 and 580).

Figure B3. Relative response rate in the lean and rich schedule components during all sessions of Response-Elimination and Resurgence phases of Experiment 6, for Pigeons 1 and 2. Vertical lines in each graph separate Response-Elimination and the Resurgence-phase sessions. Other details as in Figure B1. 
Figure B4. Residuals (obtained - predicted) of fits of Equation A3 to data from all sessions of Response-Elimination and Resurgence phases of Experiment 6, for Pigeons 1 and 2. In each graph, dashed vertical lines separate Response-Elimination and the Resurgence-phase sessions. Other details as in Figure B2. 


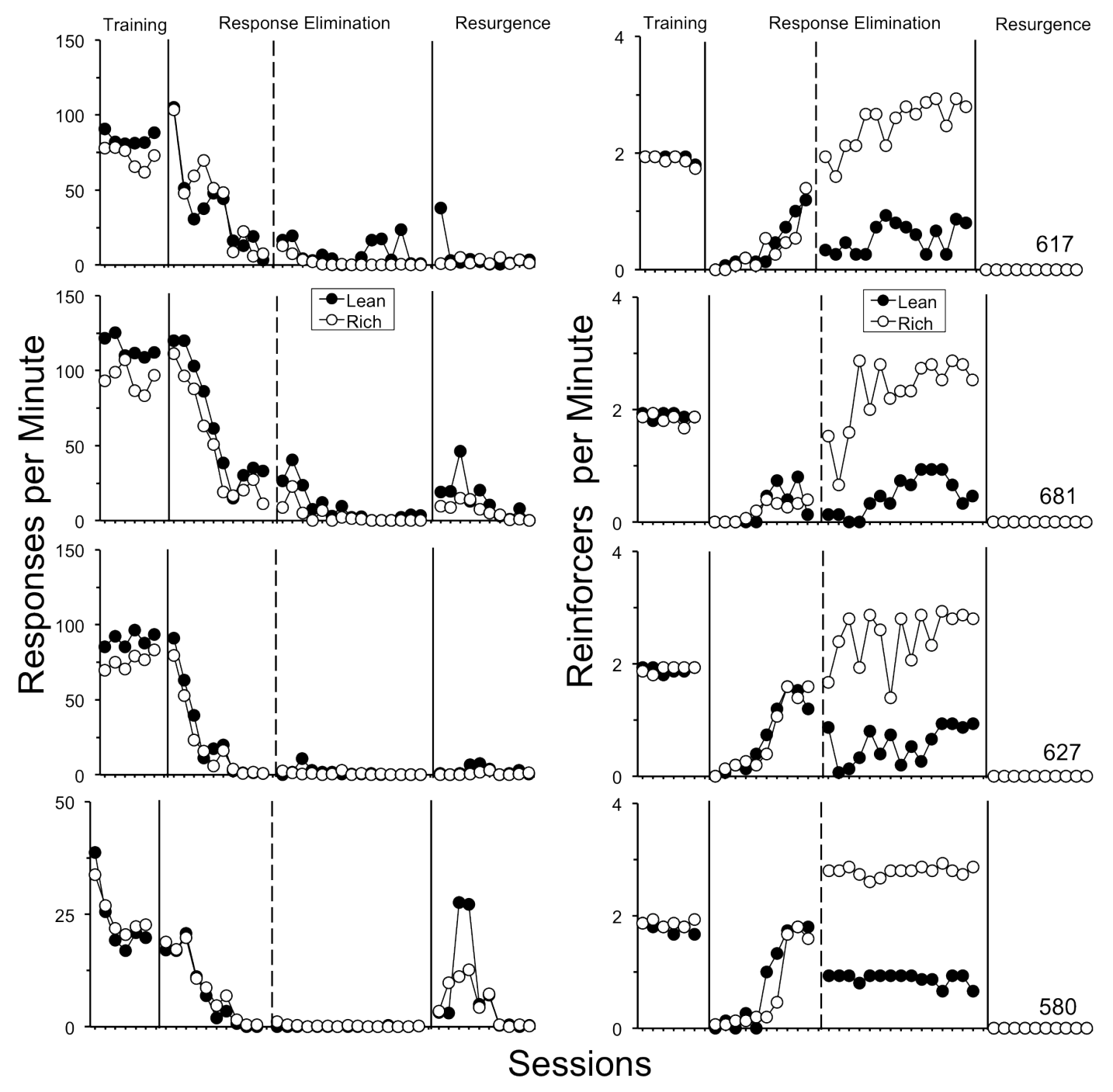




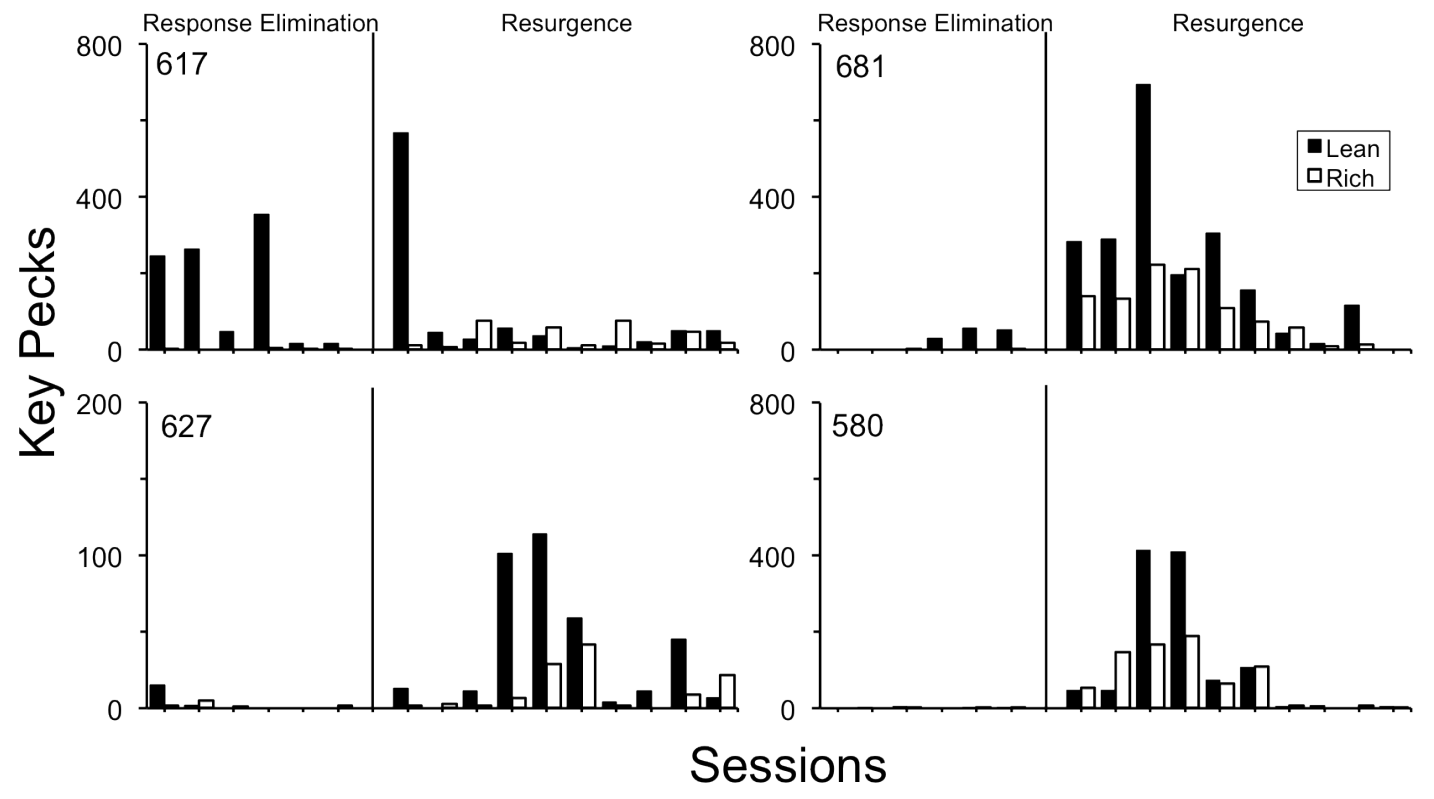




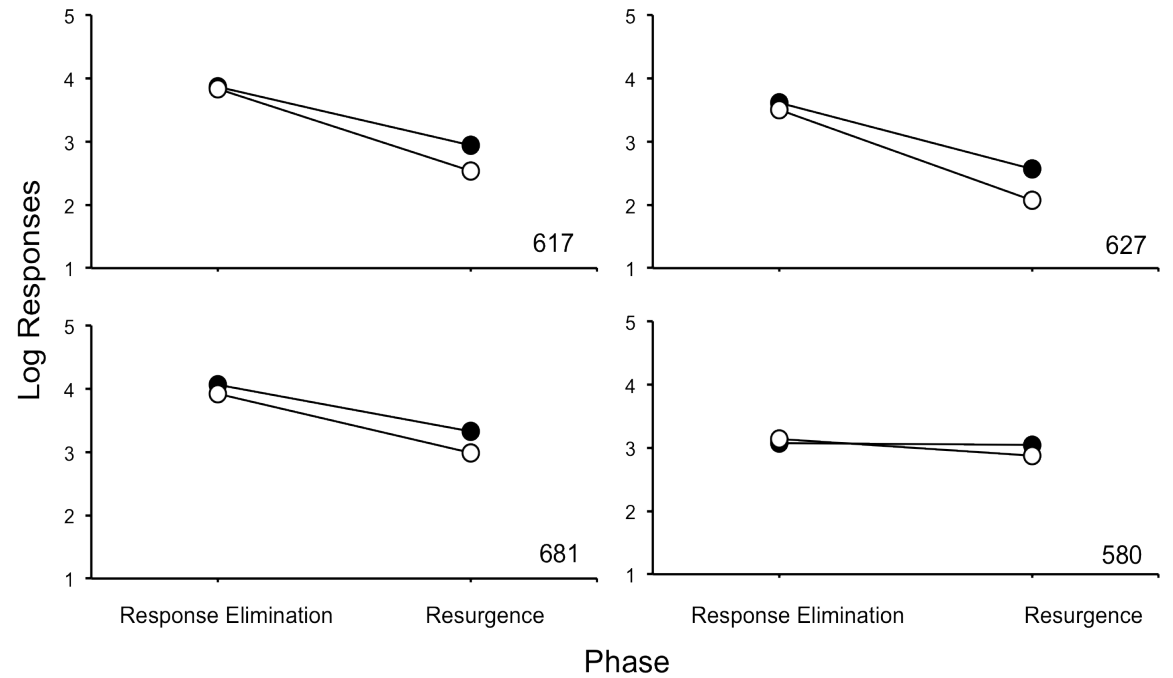


First Component in Session

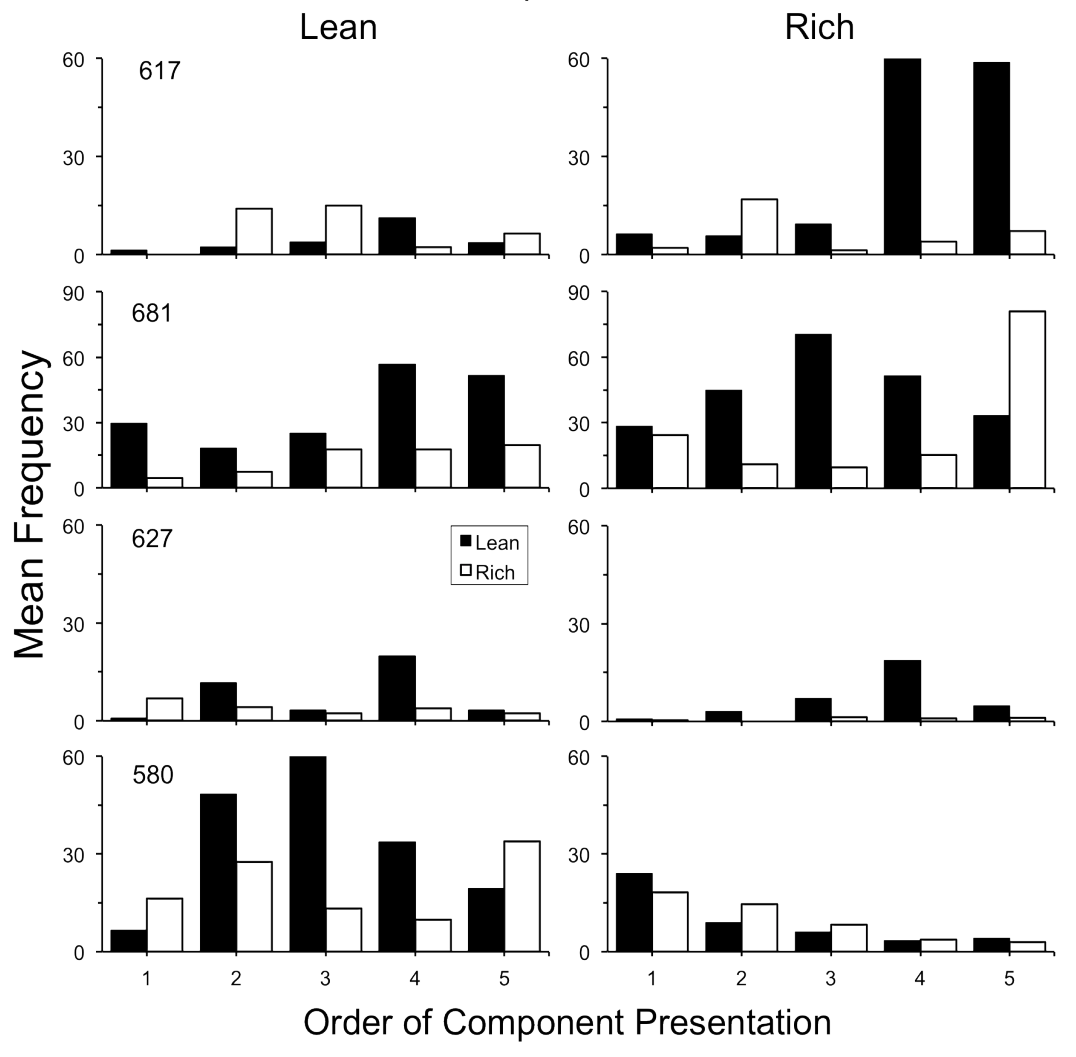




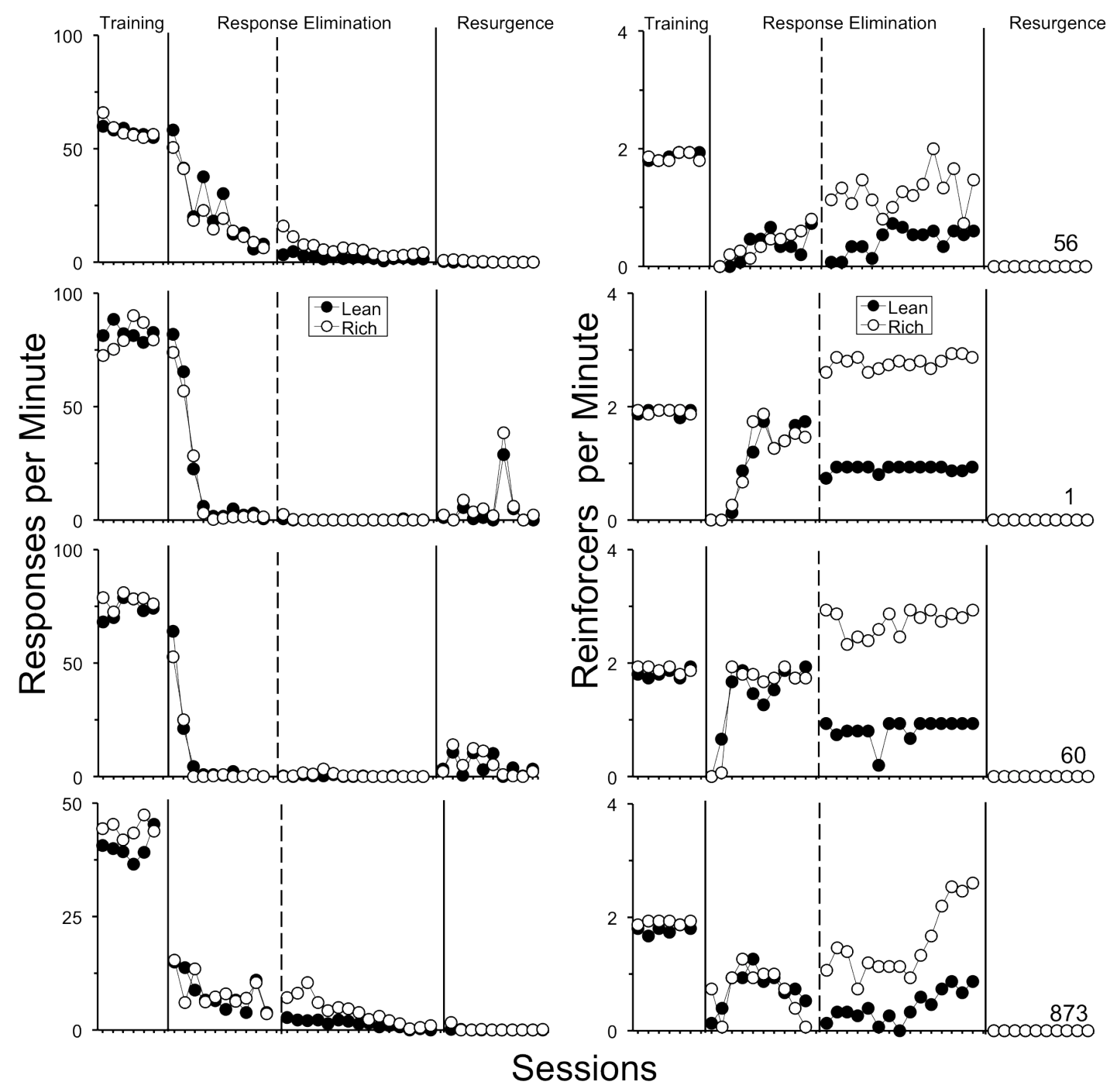




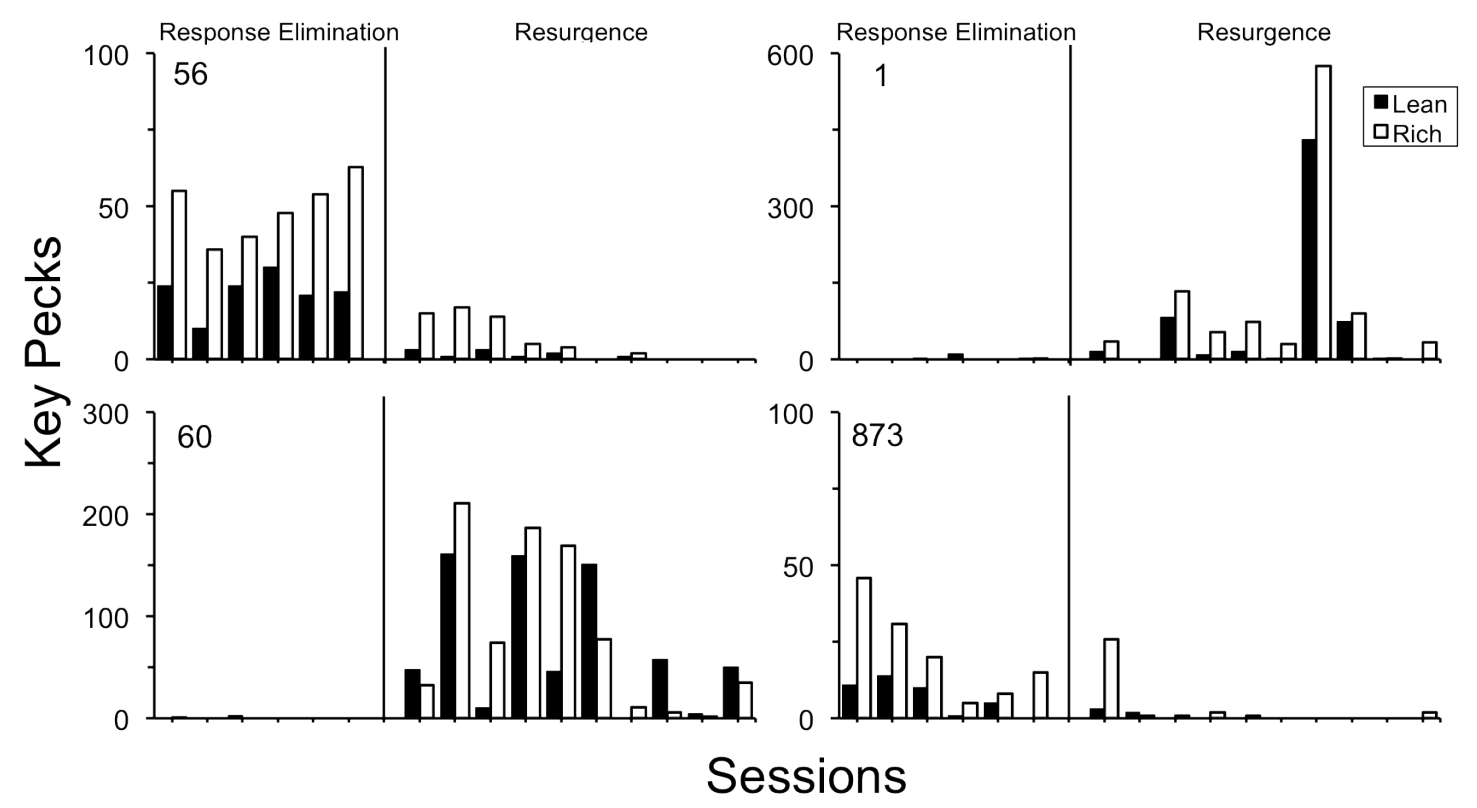




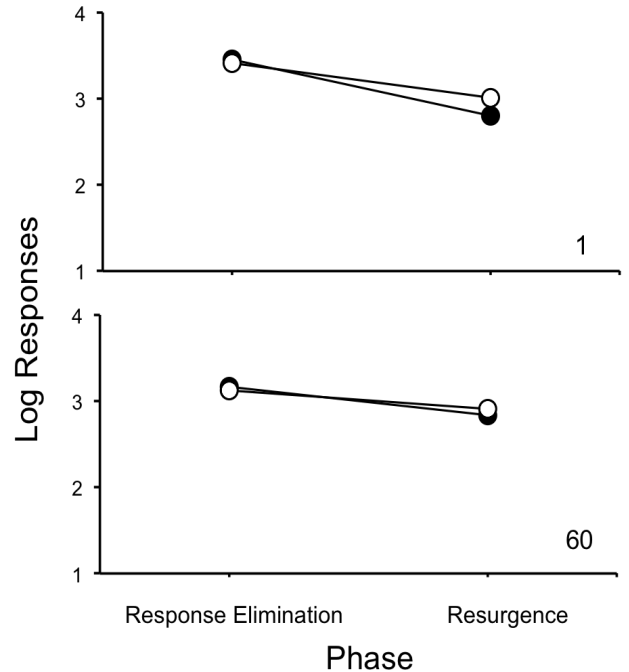




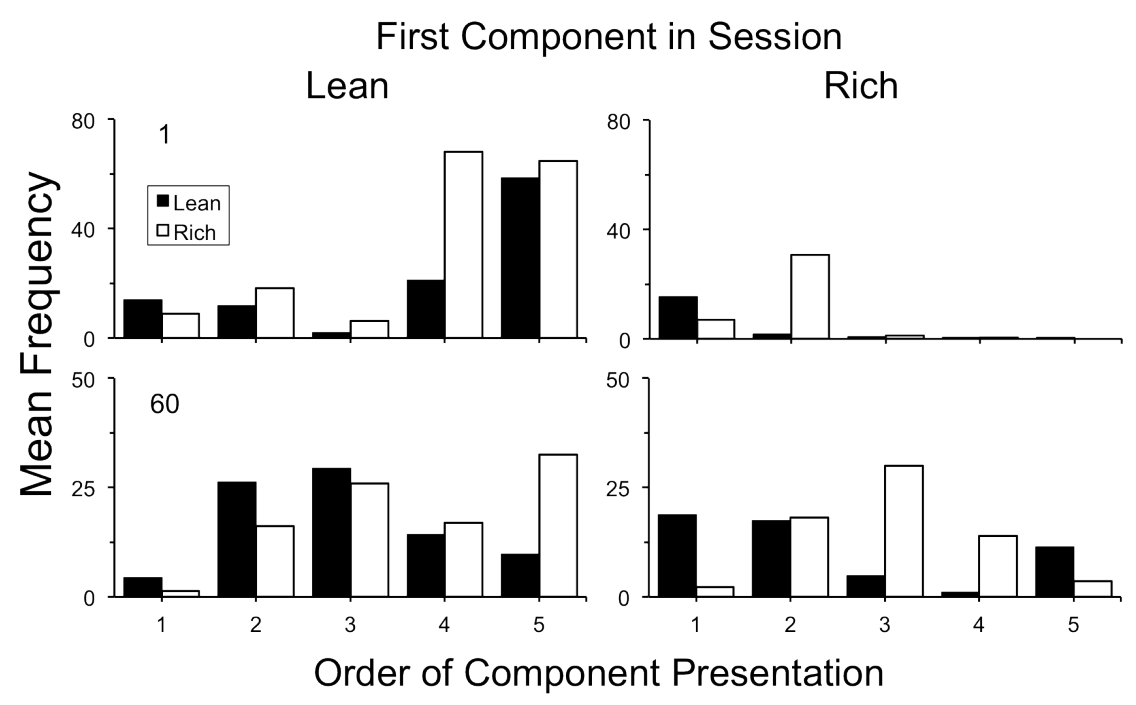




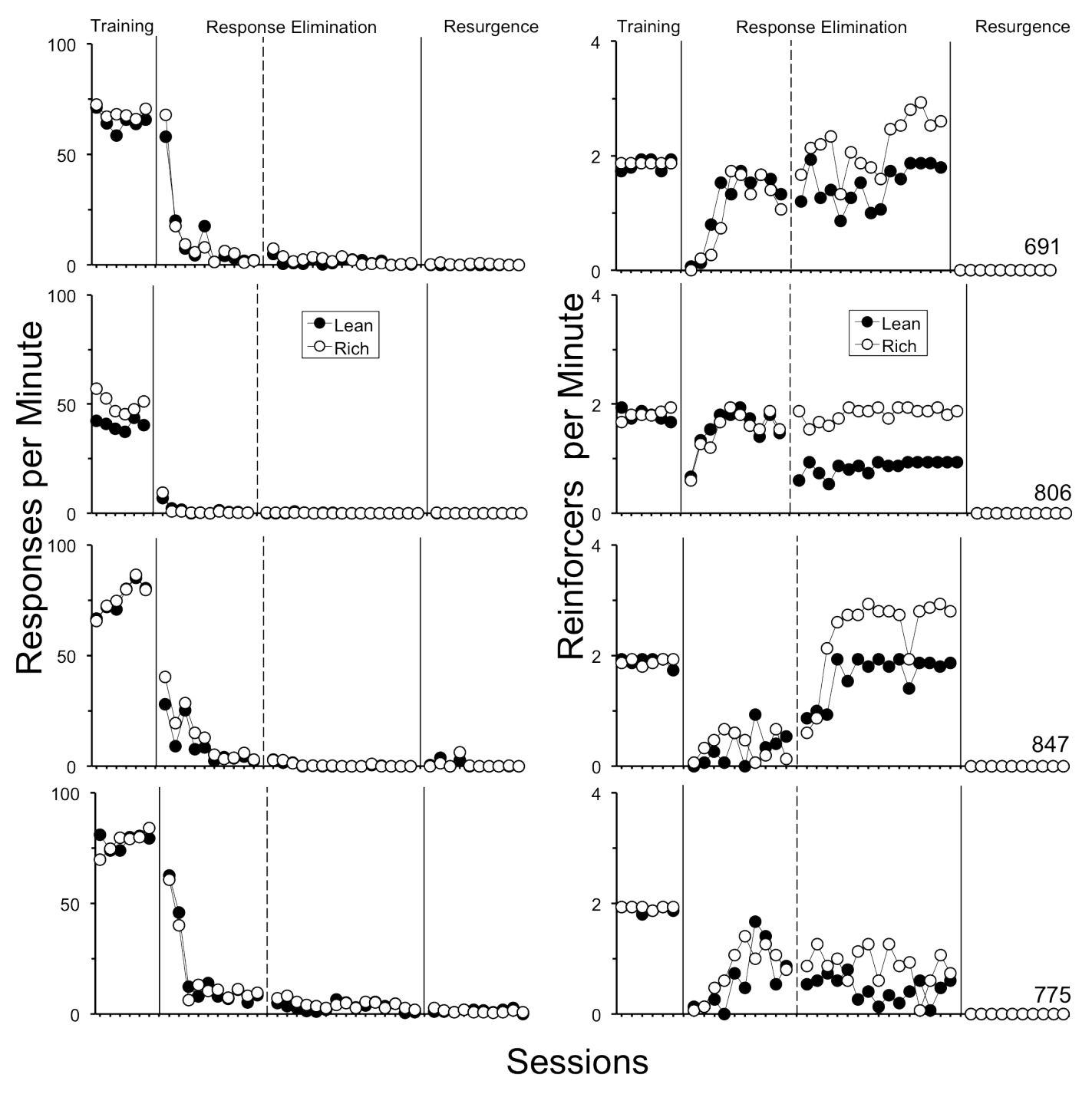




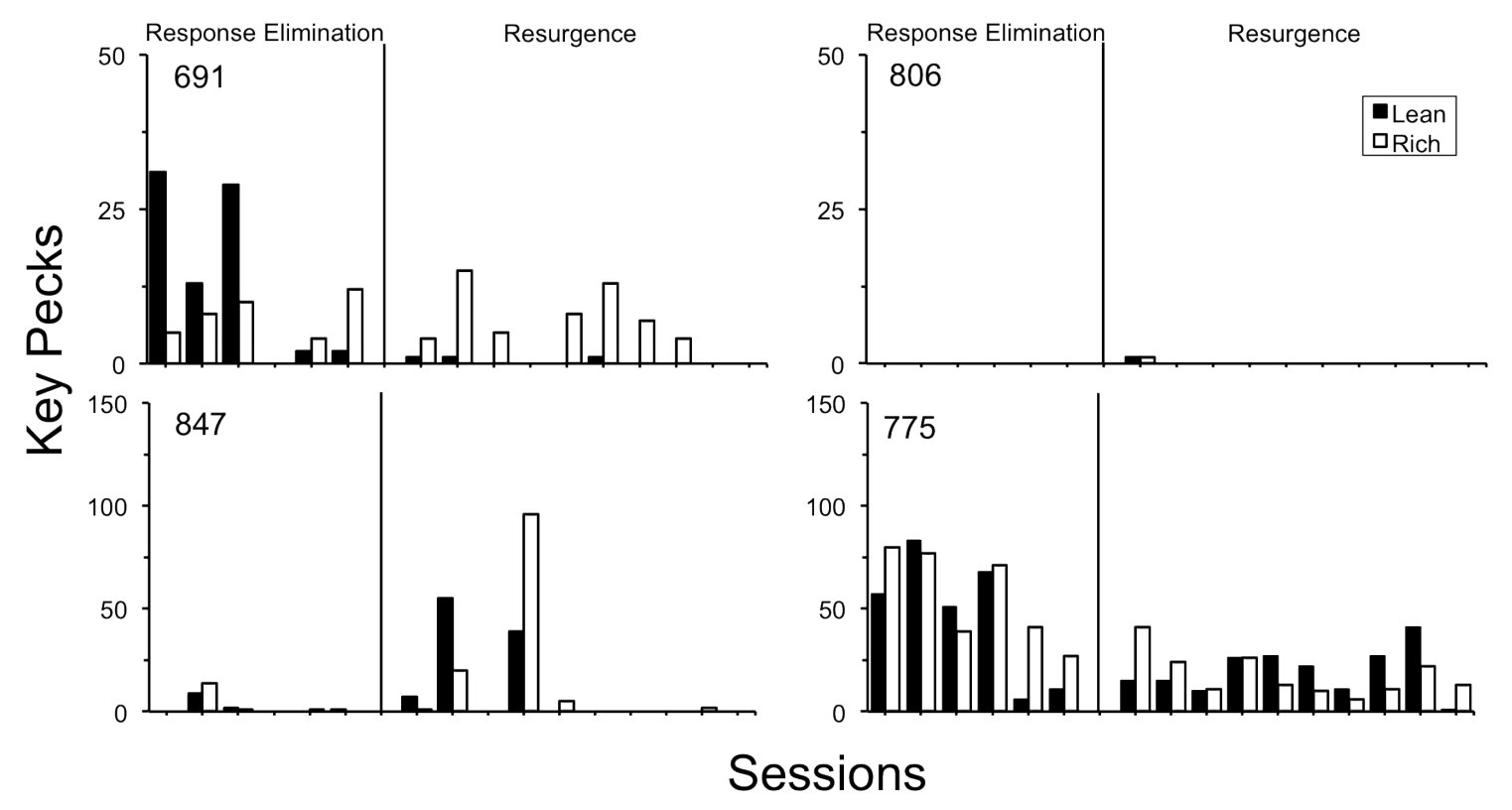




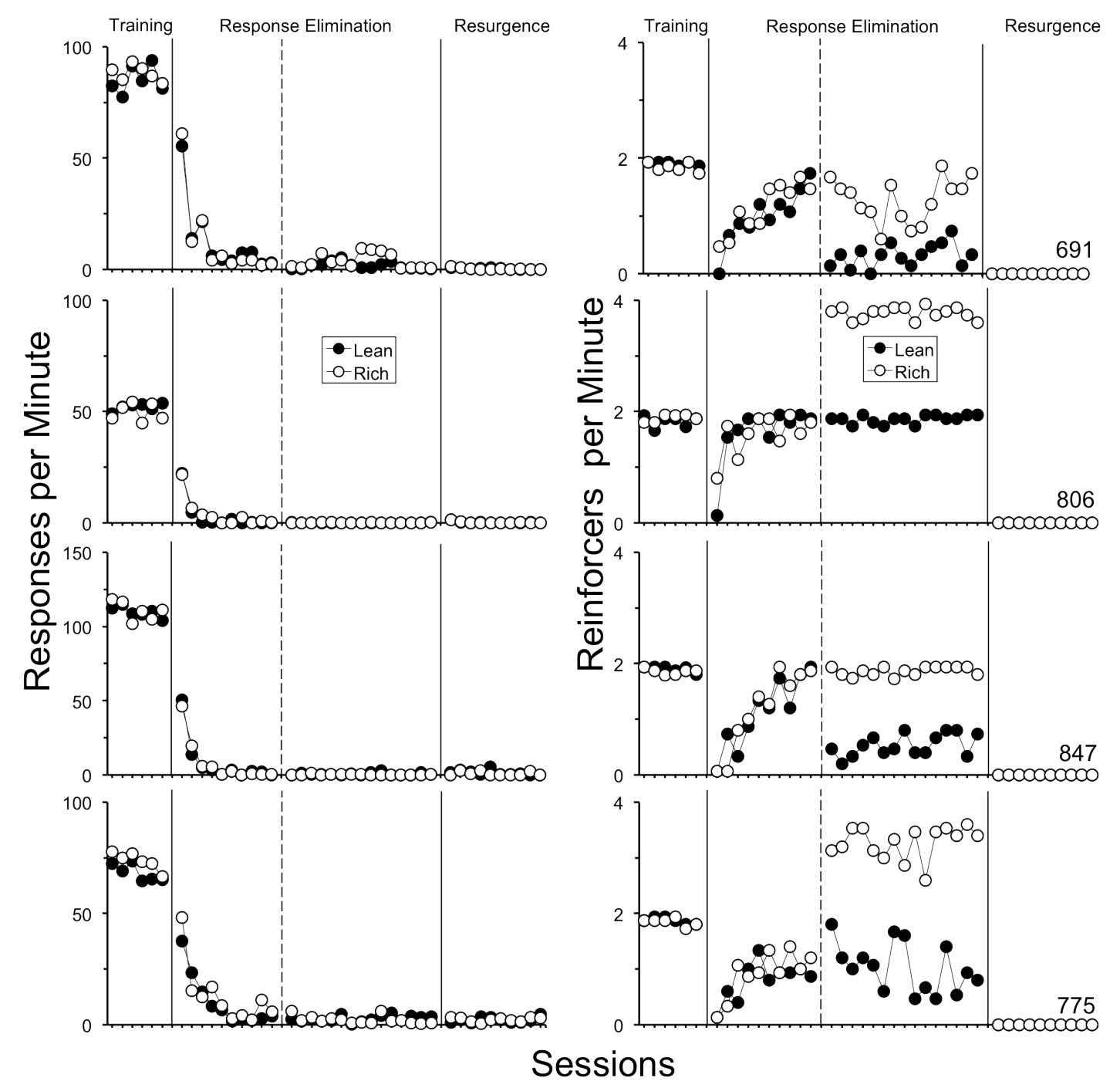




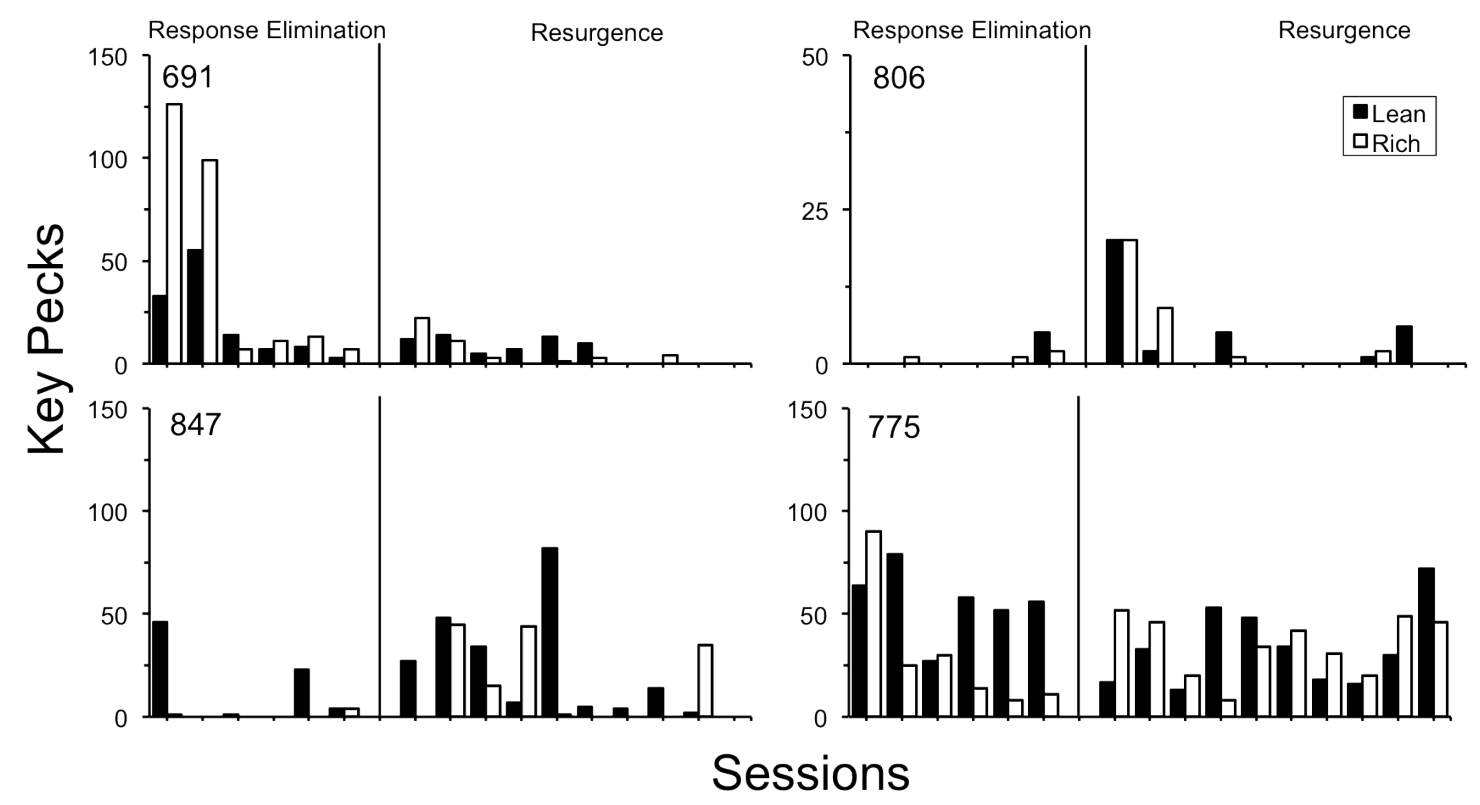




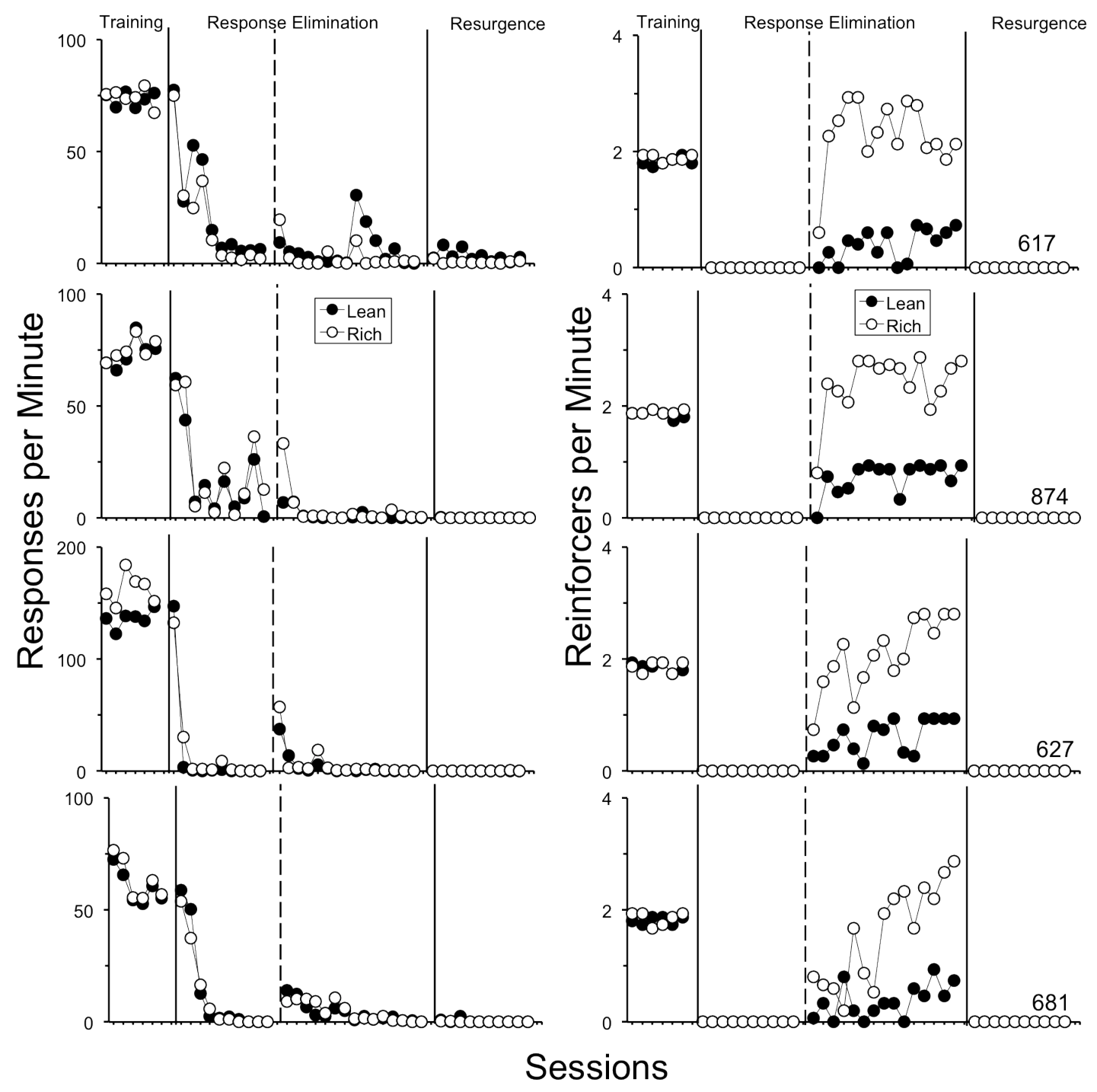




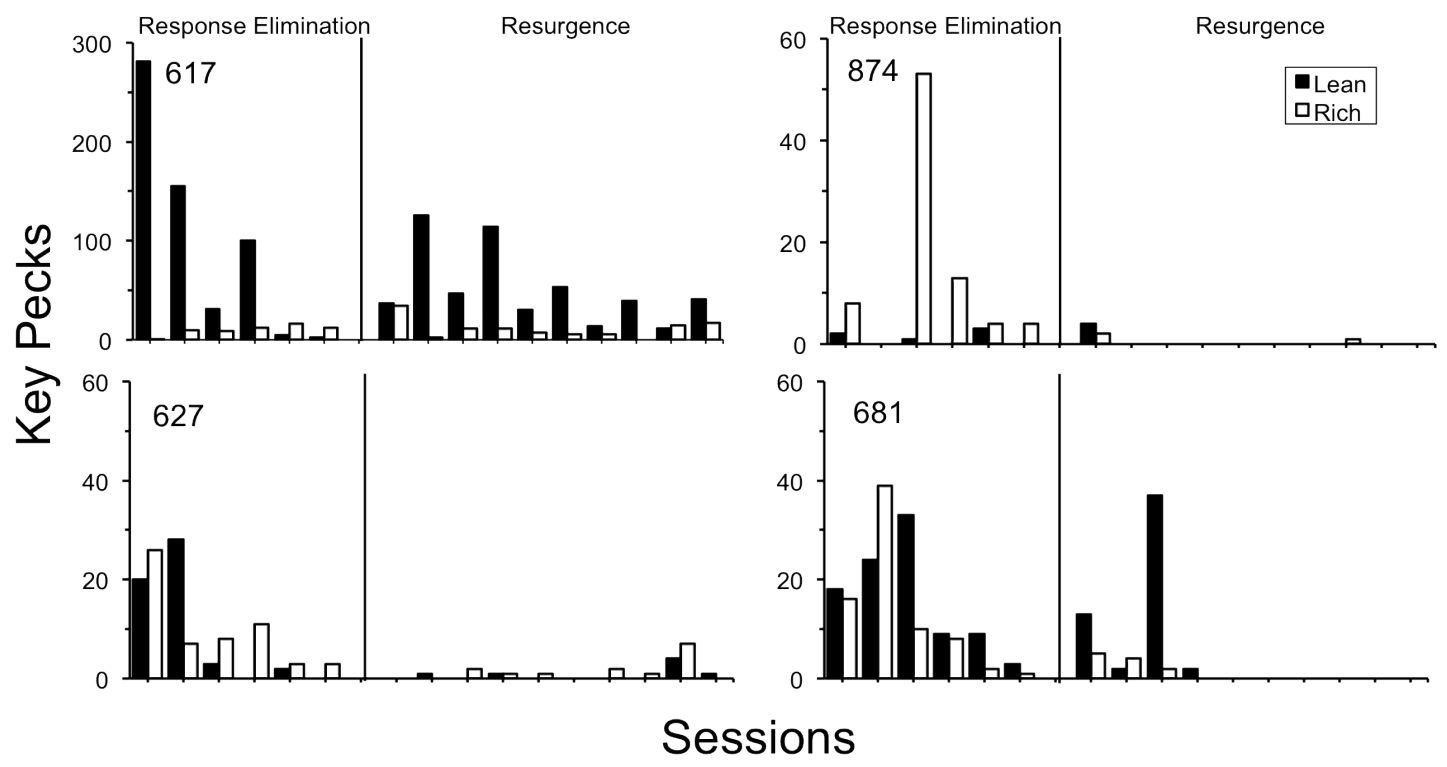




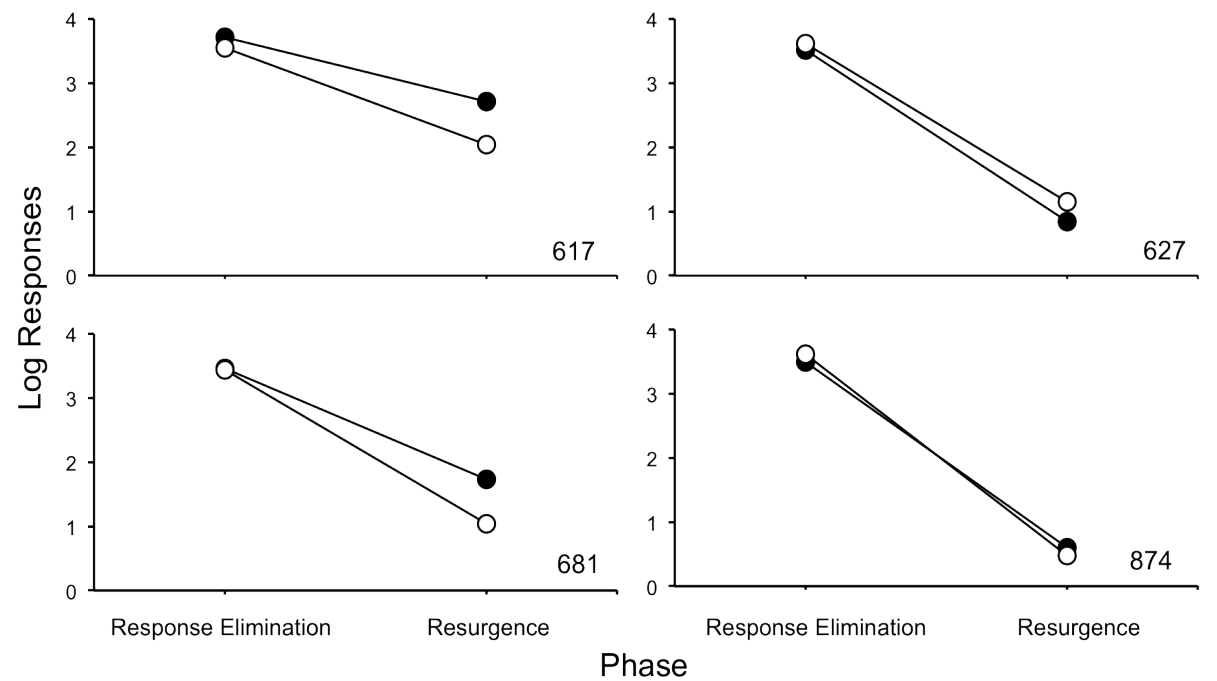




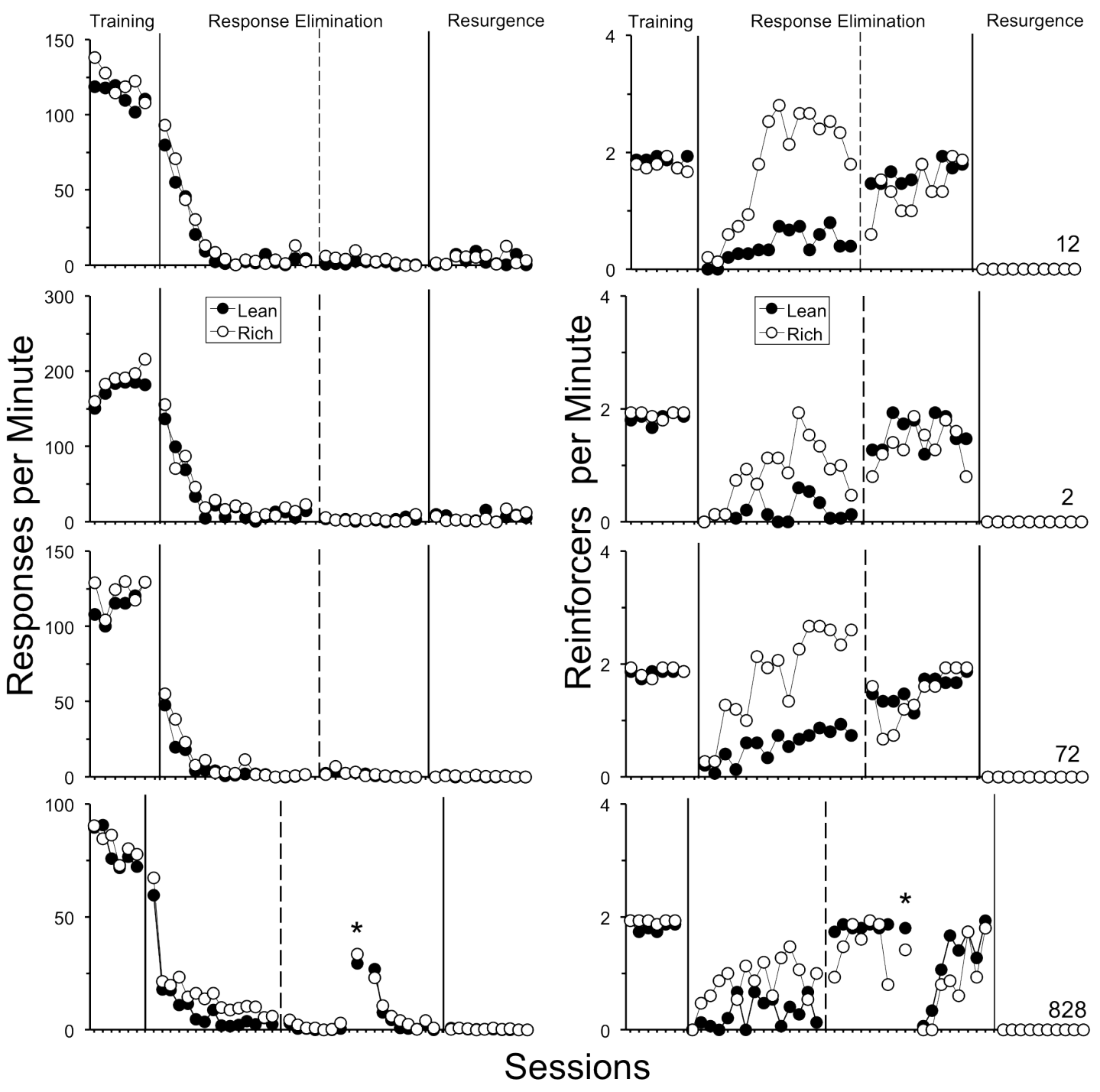




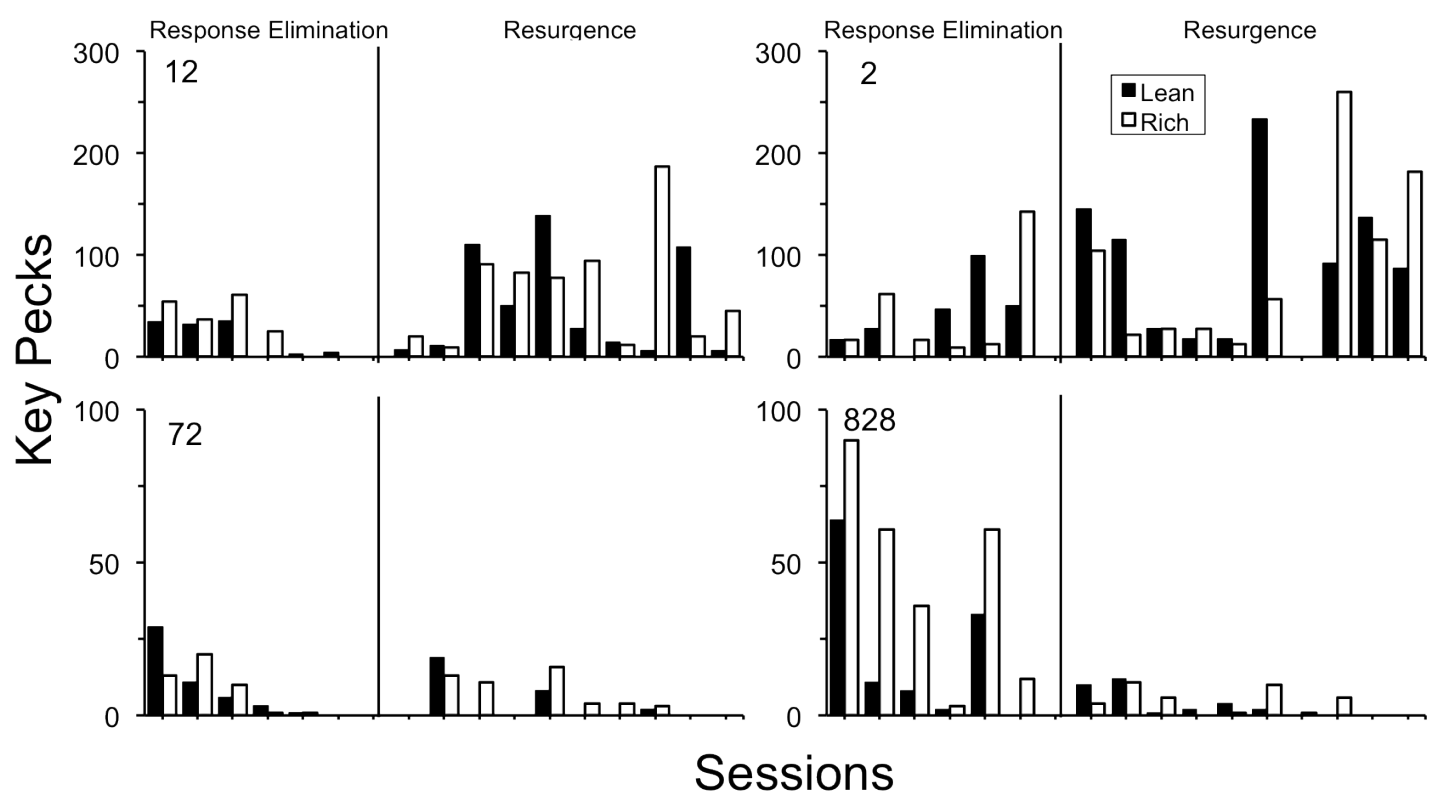



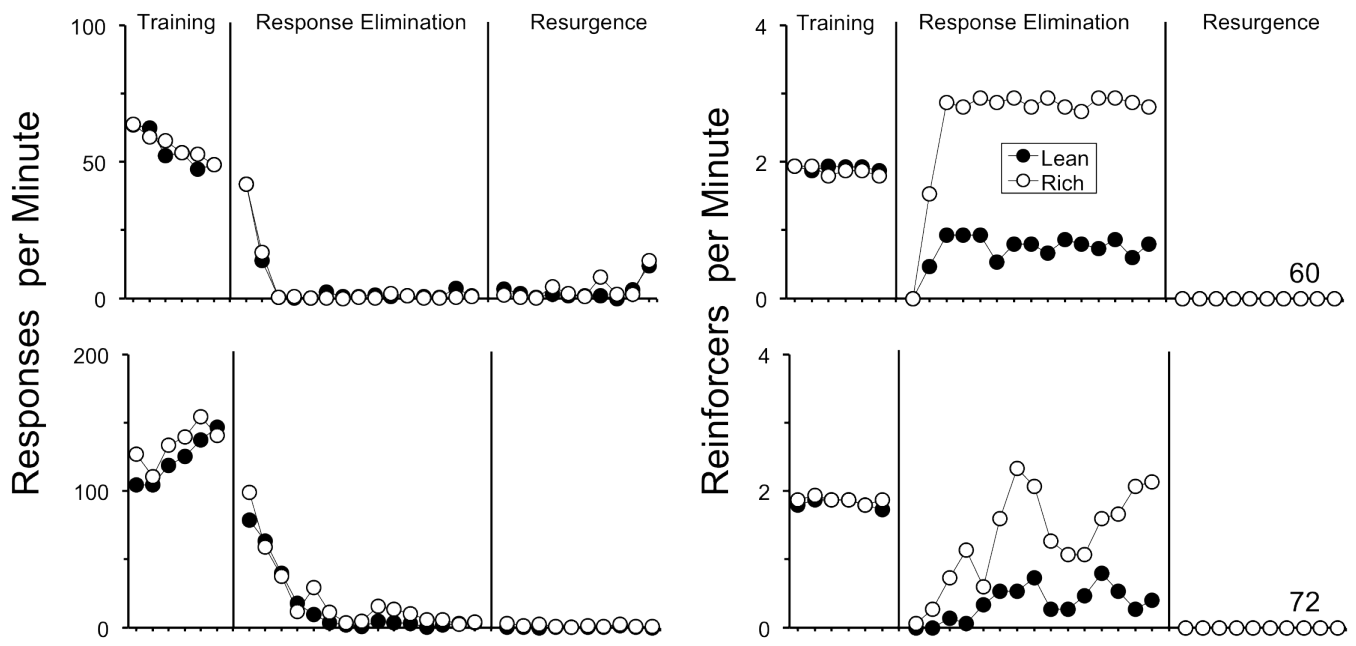

Sessions 


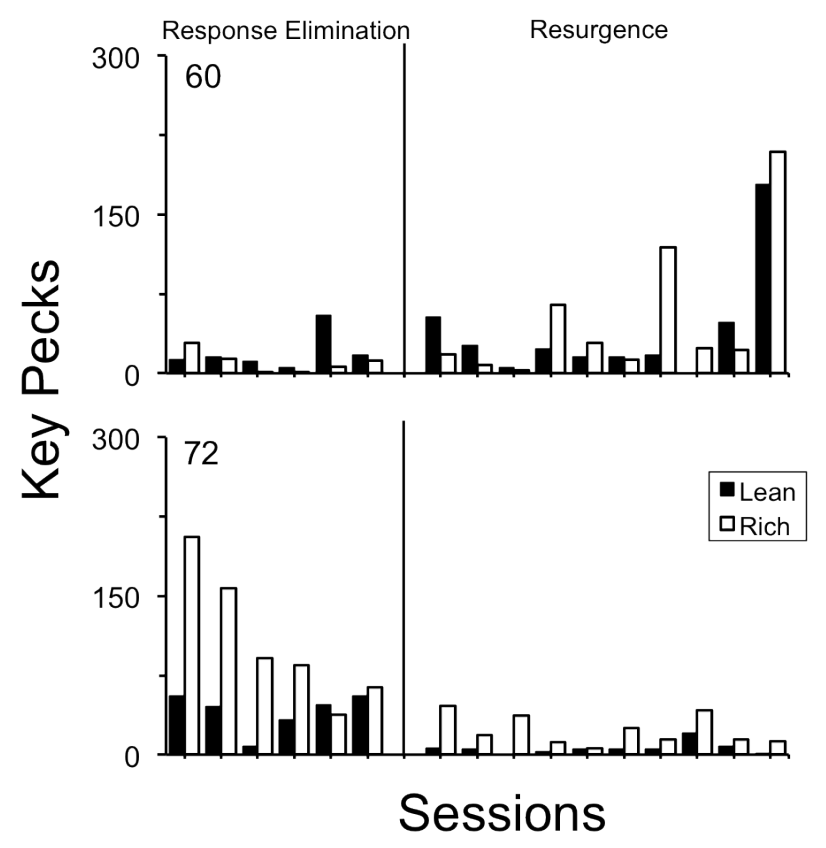



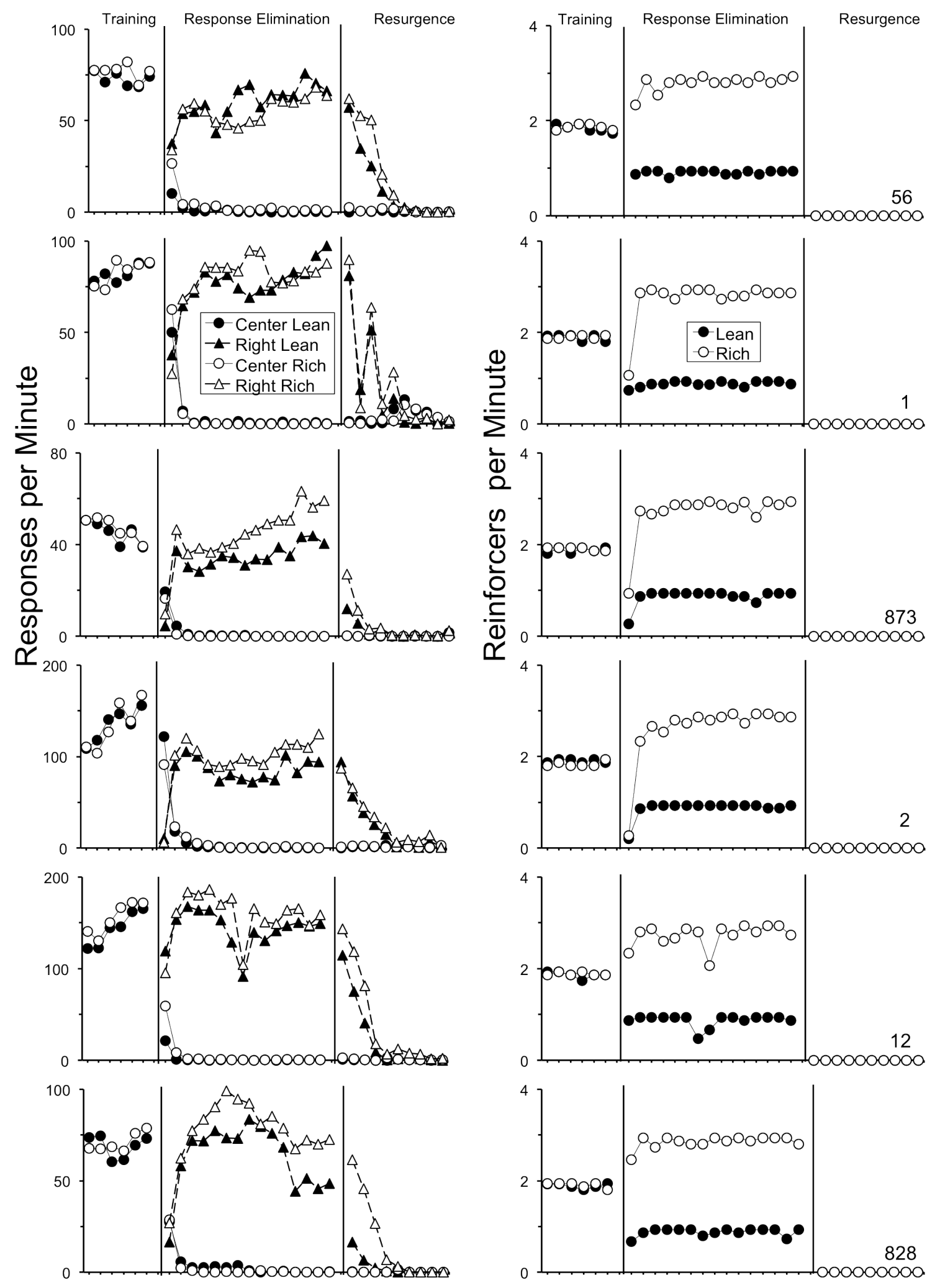

Sessions 


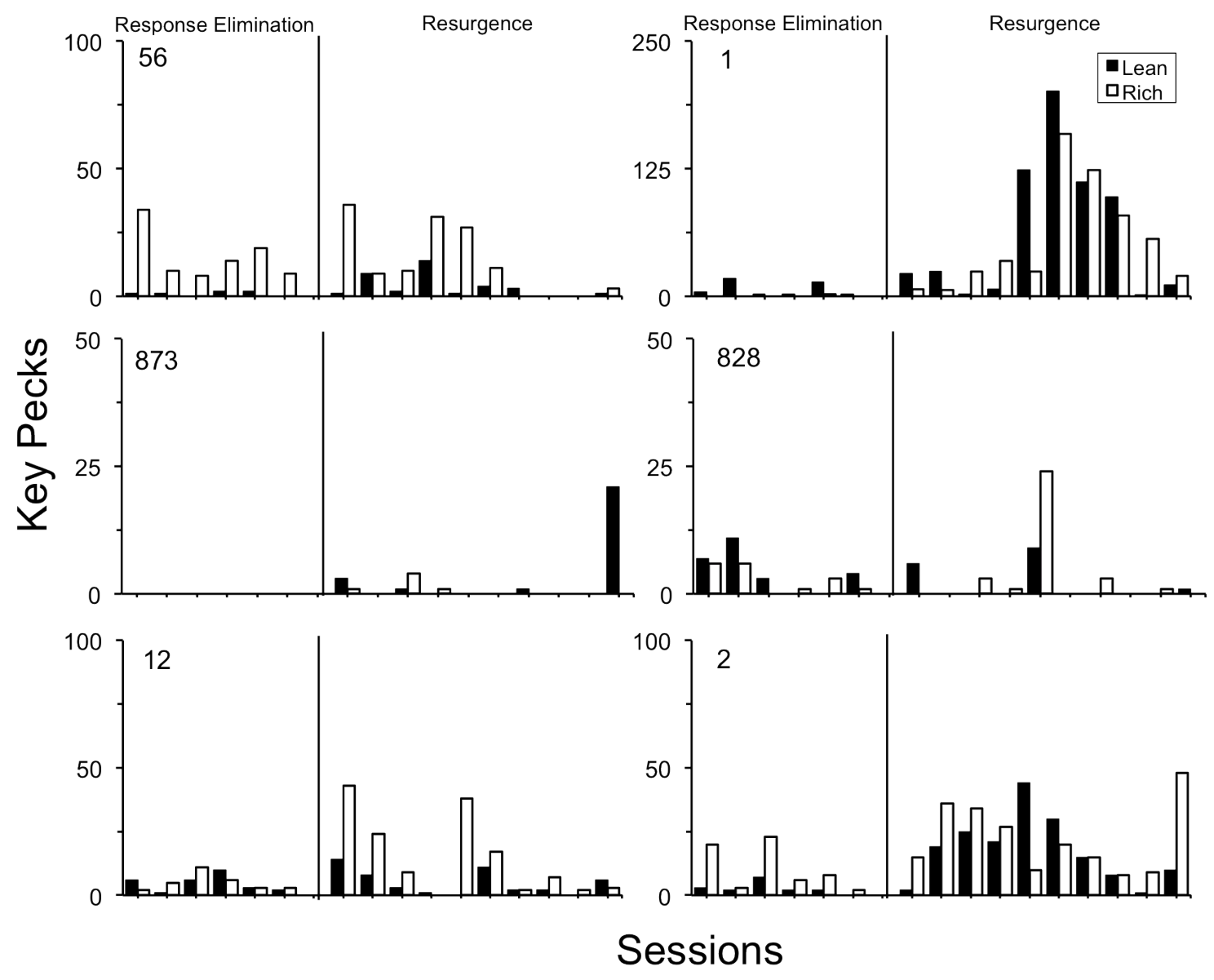




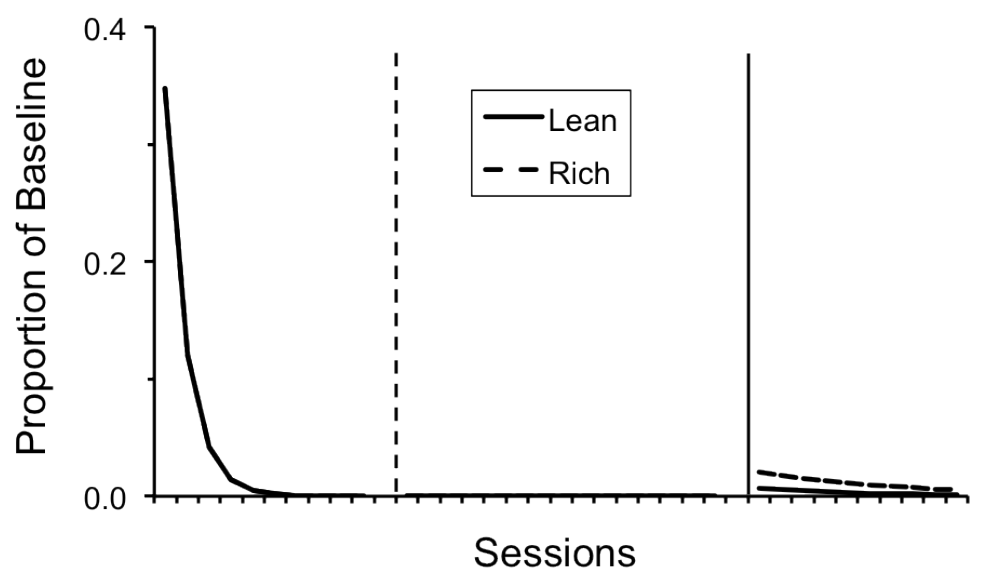




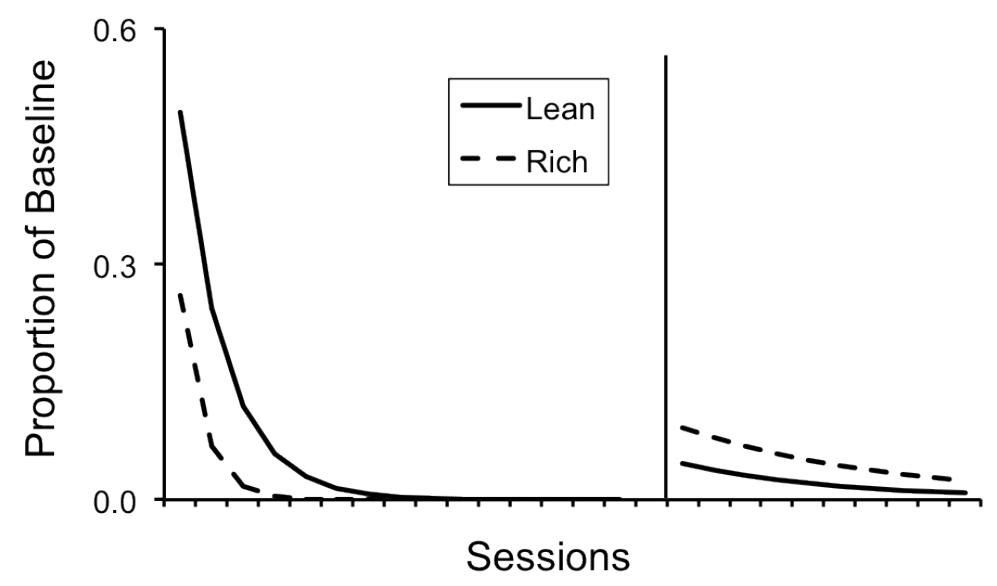




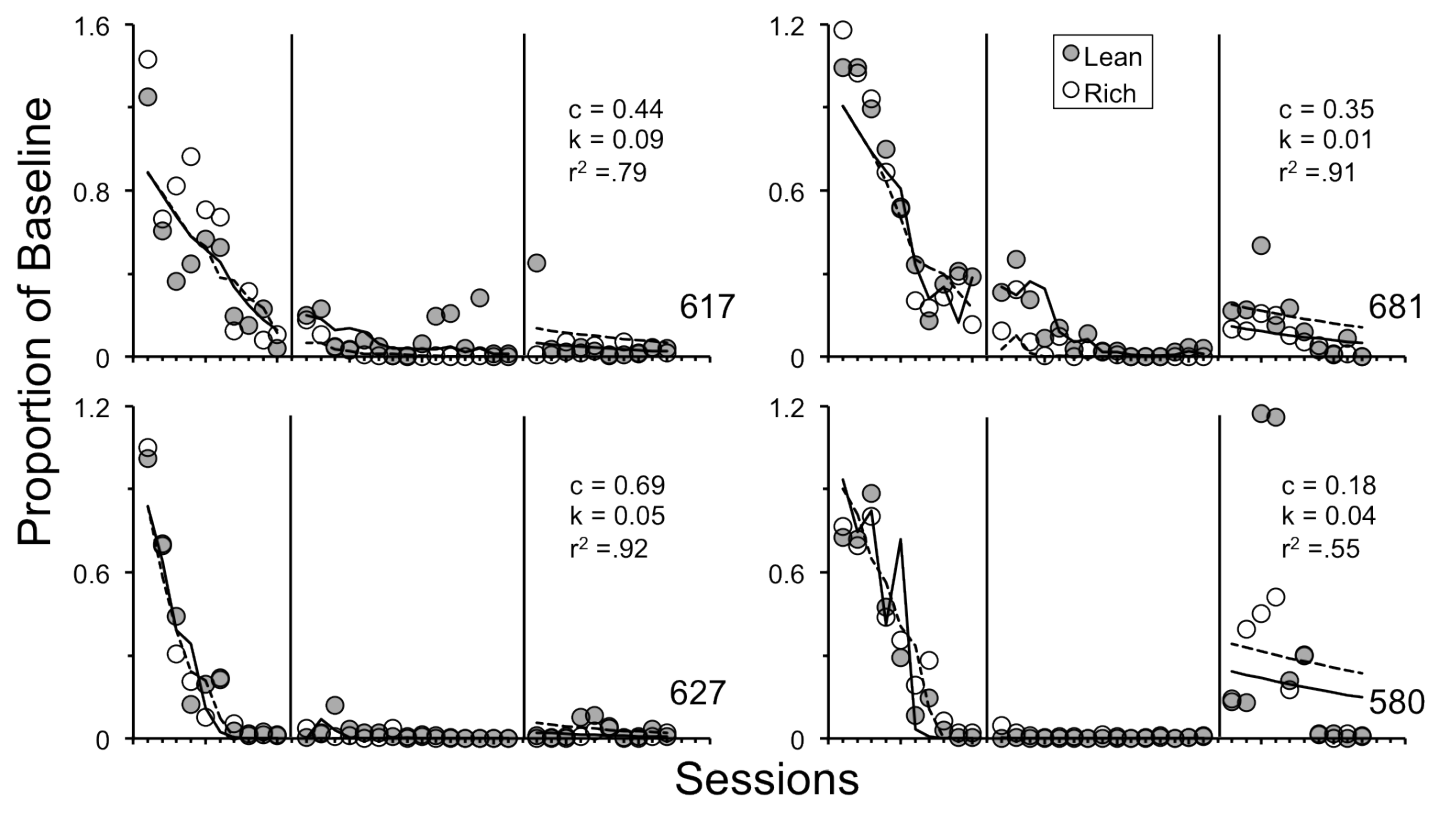




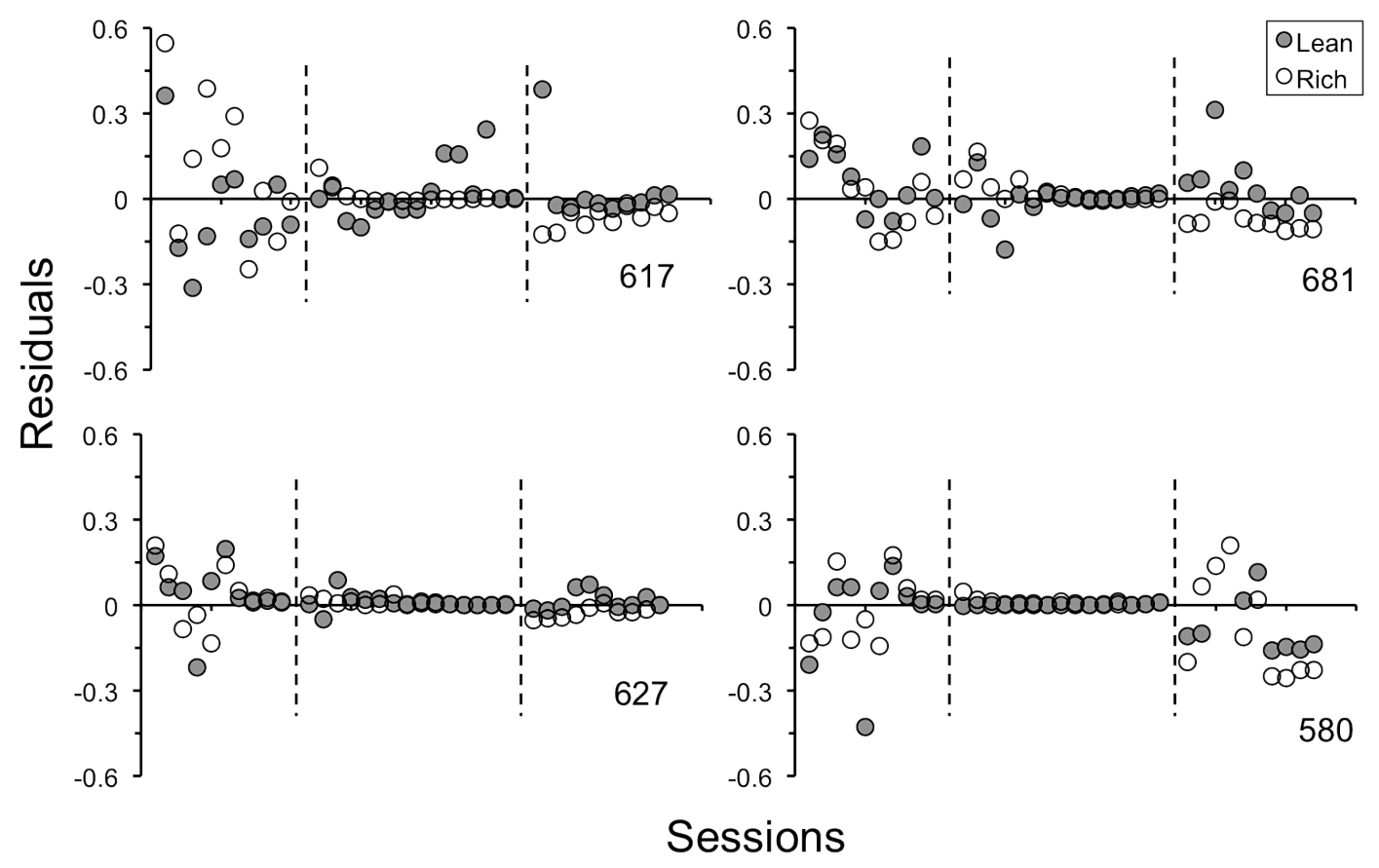




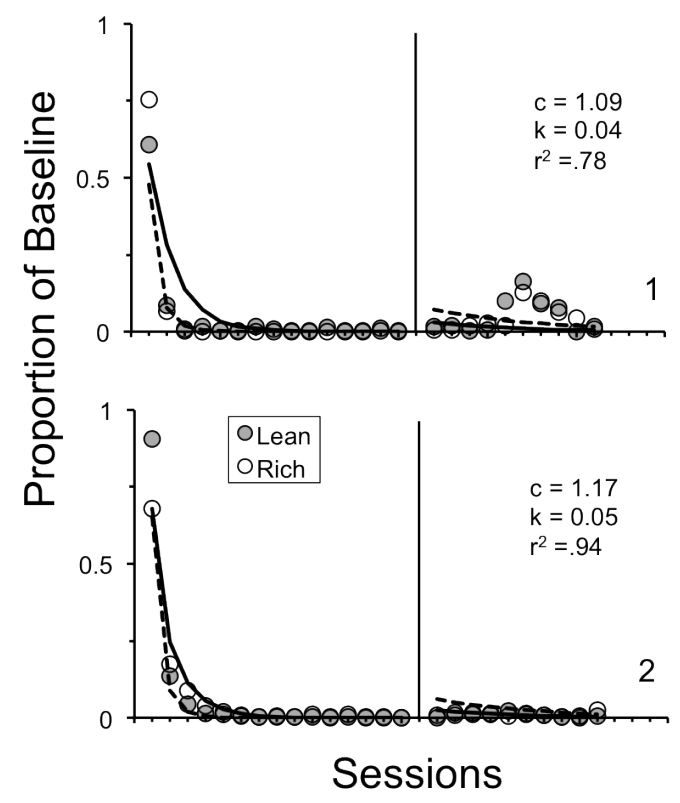




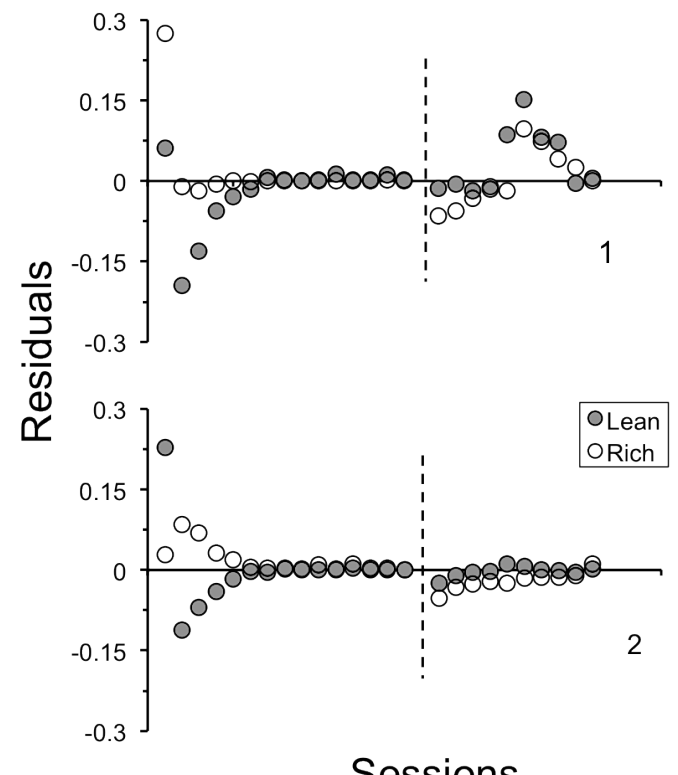

Sessions

John $\mathrm{H}$.

Hent

Hagen 